\title{
Teenage Pregnancy And Sexually Transmitted Infection Prevention: A Pilot Study Exploring Knowledge And Reported Behaviors
}

\author{
Brooke A. Flinders, Miami University, USA
}

Katelyn Gilb, Miami University, USA

Tricia Neu, Miami University, USA

\begin{abstract}
The Teen Pregnancy Prevention Program (TPP) is administered by the Office of Adolescent Health, with a goal of addressing the rising teen pregnancy rates in the United States. One TPP initiative includes the replication of evidence-based program models that seek to prevent teen pregnancies and sexually transmitted infections (Department of Health and Human Services, 2014). FOCUS Butler County, in Southwestern Ohio, is one such women's health program. In a pilot study, conducted between March and August 2011, 96 female participants between the ages of 16 and 23 were surveyed to capture a "snapshot" of their baseline knowledge and behaviors. Following their completion of the questionnaire, respondents participated in eight hours of evidenced-based, comprehensive sexual education. Four months post-intervention (n=49) and 12 months post-intervention $(n=28)$, participants were re-surveyed to assess knowledge and behaviors. This longitudinal, observational study explored the frequency and means of reported responses. For further analysis, questions were assigned to one of ten categories: 1) demographics/history, 2) knowledge-based, 3) reported behaviors, 4) opinions, 5) perceived opinions of friends, 6) intentions for future practice, 7) perception of risk, 8) attitudes regarding condom use, 9) attitudes related to alcohol use, and 10) perception of comfort related to health seeking behaviors. Significant findings were noted in the category of knowledge-based questions.
\end{abstract}

Keywords: Teen Pregnancy Prevention; Sexually Transmitted Infection; Women's Health; Sex Education

\section{INTRODUCTION}

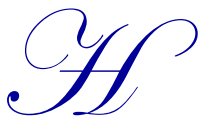

ealthy People 2020 (U.S. Department of Health and Human Services, 2013) has identified several important goals related to family planning for females, including to: 1) increase the proportion of females at risk of unintended pregnancy who used contraception at most recent sexual intercourse, 2) reduce pregnancy rates among adolescent females, 3) increase the proportion of adolescents aged 17 years and under who have never had sexual intercourse, 4) increase the proportion of sexually active adolescents aged 15 to 19 years who use contraception that both effectively prevents pregnancy and provides barrier protection against disease, and 5) increase the proportion of adolescents who received formal instruction on reproductive health topics before they were 18 years old.

In alignment with these national goals, the Office of Adolescent Health (OAH) was established, in 2010, to support and evaluate evidence-based teen pregnancy prevention (TPP) programs and to implement the Pregnancy Assistance Fund. One set of strategic priorities for OAH is to identify, promote, and support a range of evidencebased approaches to enhance adolescent health, such as behavioral risk reduction, positive youth development, program and policy interventions, and recommended clinical preventive care for adolescents and young adults (USDHHS 2013). OAH is also charged with communicating adolescent health information to health professionals and other youth-serving entities (USDHHS, 2013). 
FOCUS was one of thirty evidence-based programs approved for replication (Sociometrics, 2005). The educational program was originally conducted with young women in the US Marine Corps and stated goals of the FOCUS curriculum included primary pregnancy prevention and STD/HIV/AIDS prevention. The original study sample was comprised of 2,157 young women, aged 17 or older; approximately $90 \%$ of participants were younger than 22 (Boyer et al., 2005).

FOCUS is now being delivered to females, 16-19 years of age, in schools and other community-based settings in Butler County, Ohio, through TPP funding and an on-going partnership between the YWCA Hamilton and Miami University's Department of Nursing. Collaborative efforts have enabled these two agencies to meet the needs of an otherwise underserved population.

\section{BACKGROUND}

In 2010, before the FOCUS Program began, a needs assessment for Butler County and the surrounding region was completed. During this time, it was discovered that, of the 88 counties in Ohio, Butler County ranked $50^{\text {th }}$ in "overall health outcomes" and $48^{\text {th }}$ in "overall health factors" (with a 1 rating being "best"). As a means of providing context, selected findings from the 2010 needs assessment will be highlighted. Table 1: Butler County Health Statistics lists relevant health factors and outcomes, as they compared to the "target rates" and Ohio's overall rates (County Health Rankings and Roadmaps).

Table 1: Butler County Health Statistics

\begin{tabular}{|l|c|c|c|}
\hline \multicolumn{1}{|c|}{ Health Factor } & Butler County & Target & Ohio \\
\hline Teen births; \#/1K & 37 & 22 & 41 \\
\hline Chlamydia; \#/100K & 334 & 75 & 413 \\
\hline Binge drinking, \% & 18 & 10 & 16 \\
\hline Low birth weight, \% & 7.5 & 6.4 & 8.4 \\
\hline High school graduation, \% & 78 & 93 & 79 \\
\hline $\begin{array}{l}\text { Income inequity coefficient } \\
* 100=\text { all income in county in one household. } \\
* 0=\text { all income equally distributed }\end{array}$ & 42 & 37 & 45 \\
\hline
\end{tabular}

State-level data was evaluated in order to obtain perspective on sexually transmitted infection (STI) rates. In a list of the top ten states in the US ranked by rate of reported STI cases in 2008, Ohio ranked 10th for gonorrhea with 146.5 per 100,000 (Avert, 2010). The U.S. Department of Health and Human Services Community Health Status Indicators (2009) compared U.S. counties to "peer counties" and to the U.S. median (Table 2). It was noted that Butler County is very similar to identified peers, but that the statistics fall on the high end for both infant and neonatal mortality rates.

Table 2: Health Statistics For Butler County As Compared To "Peer" Counties

\begin{tabular}{|l|c|c|c|}
\hline \multicolumn{1}{|c|}{ Health Status Indicators } & Butler County \% & Peer Counties \% & US Median \% \\
\hline Births to Women <18 Years of age & 3 & $1-4$ & 3 \\
\hline Births to Unmarried Women & 32 & $17-35$ & 35 \\
\hline Premature Births & 11 & $9-13$ & 12 \\
\hline Very Low Birth Weight & 2 & $1-2$ & 2 \\
\hline No care 1 st $^{\text {Trimester }}$ & 13 & $8-18$ & 16 \\
\hline $\begin{array}{l}\text { Infant Mortality per 1000 live } \\
\text { births }\end{array}$ & 8 & $4-8$ & 7 \\
\hline White Infants & 8 & $3-6$ & 6 \\
\hline Black Infants & 11 & $0-17$ & 14 \\
\hline Hispanic infants & 7 & $2-9$ & 6 \\
\hline Neonatal mortality/1000 infants & 6 & $3-6$ & 5 \\
\hline
\end{tabular}




\section{PURPOSE}

The purposes of this pilot study were to 1) gather data on baseline knowledge and behaviors, related to sexual health, in a target population of 16-23 year old females in southern Ohio and 2) examine knowledge and reported behaviors at 4-months and 12-months post intervention, following teen participation in an evidence-based TPP Program.

\section{METHODS}

This was a longitudinal, observational study. Statistical Analysis Software (SAS) was used to find frequency and means of responses. Questions were assigned to one of ten basic categories: 1) demographics/history, 2) knowledge-based, 3) reported behaviors, 4) opinions, 5) perceived opinions of friends, 6) intentions for future practice, 7) perception of risk, 8) attitudes regarding condom use, 9) attitudes related to alcohol use, and 10) perception of comfort related to health seeking behaviors. The question categories were then analyzed, using the mixed procedure, to determine the proportions of change between baseline surveys and 12-month follow-up surveys. Methods used in the knowledge-based category are detailed within those findings.

\section{PROCEDURES}

\section{Search}

The recruitment and selection of subjects occurred through communication with local schools and agencies and requests for female volunteers, 16-23 years of age. The YWCA Project Director negotiated agency contracts so that administrators of community partners were fully aware of the FOCUS content and materials used.

\section{Sampling}

The study sample included 96 females, ages 16-23 years, from Miami University, a local high school, a vocational school and community organization located in Butler County, Ohio. The FOCUS curriculum was implemented at: 1) Miami University in an all-day conference format, educating female participants, 16-23 years of age $(n=34), 2)$ local high school in four, two-hour sessions, educating female participants, 16-19 years of age $(n=8)$, 3 ) a community agency in four, two-hour sessions, educating female participants, 16-23 years of age $(n=12)$ and 4) local vocational school in five, one-hour sessions, educating junior and senior-level female participants $(n=39)$. The length and number of sessions were determined by the site's preferences.

\section{Inclusion And Exclusion Criteria}

There were 105 females served within our target population between March and August 2011. Of these girls, five participants were excluded from this report because their service year overlapped between the year of the study and the following year. Four participants were excluded from the study because they chose not to complete the program.

\section{Risks And Informed Consent}

The risks involved with this voluntary study were related to potential breaches of confidentiality. Safeguards to prevent privacy violations included the coding and removal of identifying information from surveys, storing confidential data in double-locked locations, and the use of the secure Checkbox system for electronic surveys. Participants and parents/guardians, for those under 18 years old, were required to sign informed consents and were given information on how to reach Miami University's Department of Nursing and the Office of Advancement of Research and Scholarship. This research was conducted in compliance with Miami University's Institutional Review Board. 


\section{Conflicts Of Interest}

There are no known conflicts of interest to report.

\section{Program Description}

Study participants were involved in a total commitment of 9 to 10 hours, including time allocated for survey completion. The FOCUS Program's curriculum takes approximately 8 hours to complete. It can be delivered in four, two-hour sessions, or in one to two days, alternately, to meet the community agency's needs. In addition to the eight hours of curriculum, three questionnaires were administered, leading to the additional one to two hours of commitment. Details on each of the four FOCUS Sessions and related Session Objectives are included in Table 3 (Sociometrics, 2005).

Table 3: Original FOCUS Session Goals And Session Objectives

\begin{tabular}{|l|}
\hline Original FOCUS Session Goals \\
\hline Session 1 \\
Increase knowledge about unintended pregnancies \\
and STDs including HIV/AIDS. Modify values, \\
beliefs, and attitudes that impact sexual behavior.
\end{tabular}
Session Objectives

1. Increase participants' awareness of how their values and attitudes shape their views of themselves as sexual beings.

2. Evaluate how participants' values impact their decisions about unplanned pregnancies and STDs.

3. Educate participants about the risks associated with unplanned pregnancies and STDs.

4. Provide basic facts on female and male reproductive anatomy and physiology.

5. Assist participants in developing and articulating their career and/or life goals.

6. Examine how reproductive health behaviors may impact participants' goals.

\section{$\underline{\text { Session } 2}$}

Increase knowledge about hormonal and barrier contraceptives. Build communication skills to prevent risky sexual behaviors and increase condom use.

1. Provide historical overview of contraceptive methods.

2. Discuss factors that influence women's decisions about contraceptive use/non-use.

3. Discuss the pros and cons of contraceptive methods.

4. Increase participants' awareness of the impacts that unplanned pregnancies may have on their personal lives, education and career. 5. Examine the range of options available in the event of an unplanned pregnancy, and sources of social support.

6. Build communication skills on sexual behavior and contraceptive use with a sexual partner.

7. Discuss participants' feelings and attitudes about contraceptive use, and communication about those topics with sexual partners.

\section{$\underline{\text { Session } 3}$}

Increase knowledge about the signs, symptoms, and consequences of STDs/HIV/AIDS. Increase knowledge about the transmission and prevention of STDs/HIV. Build communication skills to prevent STDs/HIV.

1. Describe the risks and transmission of STDs/HIV.

2. Discuss risky sexual practices.

3. Have participants assess their STD/HIV acquisition risk.

4. Increase participants' awareness of how assumptions about a potential sexual partner can influence their risk perceptions.

5. Describe signs, symptoms, and consequences of STDs/HIV/AIDS.

6. Build skills to communicate with potential partners.

7. Discuss how HIV is a reality in the community.

8. Examine the impact of HIV on those who are infected with HIV, and on their friends and families.

$\underline{\text { Session } 4}$

Modify attitudes about the effects of alcohol and its relationship to sexual risk behaviors. Build refusal communication skills. Build condom use skills. Increase awareness about how life choices can impact decision-making and health.
1. Describe the effects of alcohol use.

2. Simulate social and emotional challenges women face early in their careers.

3. Describe the steps required for proper condom use.

4. Examine the barriers to consistent condom use.

5. Provide opportunity to practice condom-use skills.

6. Build communication skills to enable participants to leave risky sexual situations.

7. Discuss appropriate time to seek reproductive health care.

8. List barriers/benefits to seeking reproductive health care. 


\section{Instruments}

The baseline and two follow-up questionnaires were established instruments used in the original FOCUS study (PASHA, 2005). Minor modifications to the curriculum and to the surveys were made with approval from program developers and the Office of Adolescent Health. These adaptations included the replacement of "military" language, such as "Medical" and "platoon", with phrases like "clinic or healthcare provider" and "class", for example. In addition, one video that was marketed for adults in the military was deleted from the program.

The baseline survey consisted of 128 questions and was completed by each participant at the site of service delivery, before the first session began. Two follow-up questionnaires were administered at four- and 12-months, post-intervention. The follow-up surveys were completed individually and were submitted by mail or via electronic survey responses, depending on the participant's preference. The 4-month survey consisted of 31 questions and the 12-month survey consisted of 131 questions. The questionnaires were adapted from the original research study, with minimal changes.

\section{FINDINGS}

\section{Demographics And Reported Histories}

Over the course of the 12-month period, only 1 of the 96 participants reported a marriage status change from unmarried to married; all other 95 participants remained single. The mean participant age was 18.45 , with a standard deviation of 2.219. Table 4 presents the reported race of participants. The original study included choices of "black", "white", or "other". These choices were left unchanged for this pilot study.

Table 4: Reported Race

\begin{tabular}{|c|c|c|}
\hline Reported Race & Frequency & Cumulative Frequency \\
\hline Black & 14 & 26 \\
\hline White & 70 & 96 \\
\hline "Other" & 12 & 12 \\
\hline
\end{tabular}

When asked on the baseline survey if they had ever had sex, 78 of 96 reported "yes" and 18 of 96 reported "no". When asked if they had ever been pregnant, 16 of 96 reported "yes" and 80 of 96 reported "no" on the baseline survey. Reports of pregnancy did not change from the baseline to the 12-month post-intervention reporting period. The mean number of reported sexual partners for our cohort was 3.96, with a standard deviation of 3.67. The mean number of reported sexual partners was 3.96, with a standard deviation of 3.67. Details about reported partner histories (steady versus casual) are included in Table 5 by survey periods.

Table 5: Reported Steady Versus Casual Partners By Survey Period

\begin{tabular}{|c|c|c|}
\hline \multirow[t]{2}{*}{ Survey Period } & \multicolumn{2}{|c|}{$\begin{array}{l}\text { Of the people you have had sex with, how many would you consider steady } \\
\text { versus casual partners? }\end{array}$} \\
\hline & Steady Partners & Casual Partners \\
\hline \multirow[t]{4}{*}{ Baseline } & Mean 2.20 & Mean 2.16 \\
\hline & Standard Deviation 2.02 & Standard Deviation 3.31 \\
\hline & Maximum 12 & Maximum 16 \\
\hline & Minimum 0 & Minimum 0 \\
\hline \multirow[t]{4}{*}{ 12-Month Follow-Up } & Mean 1.00 & Mean 0.57 \\
\hline & Standard Deviation 0.77 & Standard Deviation 1.35 \\
\hline & Maximum 3 & Maximum 6 \\
\hline & Minimum 0 & Minimum 0 \\
\hline
\end{tabular}

One question, unique to the 12- month questionnaire, was: "Do you consider your last sexual partner, since participating in the FOCUS program, to be a steady partner (someone you knew for a while and had an on-going relationship with) or a casual partner (someone you knew for a short period of time and did not have an on-going relationship with)?" Of those that answered, 19 reported their last partner to be steady and only two reported their last partner to be casual. Six others reported they had never had sex and one declined to answer. 


\section{Knowledge-Based Questions}

Many survey questions addressed knowledge regarding sexual behaviors, birth control options, and sexually transmitted infections (Appendix A). Findings regarding specific perceptions of risk and condom use (Table 6) and reported sexual practices by survey period (Table 7) are highlighted. Compared to the baseline survey, participants in the 12-month follow-up reported a higher perceived risk of having an unplanned pregnancy, a slightly lower perceived risk of getting an STI, and decreased condom use.

Table 6: Perception Of Risk And Reported Condom Use

\begin{tabular}{|l|l|c|c|}
\hline \multicolumn{1}{|c|}{ Survey Questions } & Mean & Std. Deviation \\
\hline \multirow{3}{*}{ Baseline } & $\begin{array}{l}\text { I think chance of unplanned pregnancy in first six months after } \\
\text { program is... }\end{array}$ & 10.1052632 & 16.9915334 \\
& $\begin{array}{l}\text { Chance of getting and STI after participation in program is about: } \\
\text { In general, [since program] what percent of the time do you use } \\
\text { condom when sex }\end{array}$ & 3.6489362 & 8.7445313 \\
\hline 12-Month & $\begin{array}{l}\text { I think chance of unplanned pregnancy in first six months after } \\
\text { program is... }\end{array}$ & 48.2467532 & 37.9730361 \\
$\begin{array}{l}\text { Follow- } \\
\text { Up }\end{array}$ & $\begin{array}{l}\text { Chance of getting and STI after participation in program is about: } \\
\text { In general, [since program] what percent of the time do you } \\
\text { use condom when sex }\end{array}$ & 3.3214286 & 26.1717971 \\
\hline
\end{tabular}

Table 7: Reported Sexual Practices By Period

\begin{tabular}{|l|l|c|c|}
\hline Survey Questions & Mean & Std. Deviation \\
\hline Baseline & $\begin{array}{l}\text { In past 3 months/Since program, how many people did you have } \\
\text { sex with? }\end{array}$ & 0.7956989 & 0.6520726 \\
\hline $\begin{array}{l}\text { 4-Month } \\
\text { Follow-Up }\end{array}$ & $\begin{array}{l}\text { In past 3 months/Since program, how many people did you have } \\
\text { sex with? } \\
\text { Since the program, how many times have you had sex? }\end{array}$ & 0.918367 & 0.7592961 \\
\hline $\begin{array}{l}\text { 12-Month } \\
\text { Follow-Up }\end{array}$ & $\begin{array}{l}\text { In past 3 months/Since program, how many people did you have } \\
\text { sex with? }\end{array}$ & 13.0425532 & 1.1785714 \\
\hline
\end{tabular}

The Means Procedure was used to identify means and standard deviations for both proportion of valid responses and proportion of correct responses. These Knowledge-Based proportions are presented in Table 8.

Table 8: Knowledge-Based Proportions Of Validity And Correct Responses

\begin{tabular}{|l|c|c|}
\hline \multicolumn{1}{|c|}{ Variable: Knowledge-Based } & Mean & Standard Deviation \\
\hline Proportion of Valid Responses & 0.9926857 & 0.0239758 \\
\hline Proportion of Correct Responses & 0.5989896 & 0.1730823 \\
\hline
\end{tabular}

Four elements from the SAS output, using the Mixed Procedure method, are presented below: 1) Model Information (Table 9), 2) Null Model Likelihood Ratio Test (Table 10), 3) Estimates (Table 11), and 4) Least Squares Means (Table 12).

\begin{tabular}{|l|l|}
\hline Model Information & Proportion Correct: Knowledge-Based \\
\hline Dependent Variable & Unstructured \\
\hline Covariance Structure & ID \\
\hline Subject Effect & REML \\
\hline Estimation Method & None \\
\hline Residual Variance Method & Model-Based \\
\hline Fixed Effects SE Method & Between-Within \\
\hline Degrees of Freedom Method &
\end{tabular}


Table 10: Null Model Likelihood Ratio Test

\begin{tabular}{|c|c|c|}
\hline Null Model Likelihood Ratio Test & Pr > ChiSq \\
\hline df & Chi-Square & 0.0026 \\
\hline 2 & 11.92 & \\
\hline
\end{tabular}

Table 11: Estimates

\begin{tabular}{|l|c|c|c|c|c|}
\hline Estimates & Estimate & $\begin{array}{c}\text { Standard } \\
\text { Error }\end{array}$ & df & t Value & Pr $>|\mathbf{t}|$ \\
\hline 12-Month - Baseline & $\begin{array}{c}0.09400 \\
(9.4 \%)\end{array}$ & 0.02693 & 95 & 3.49 & 0.0007 \\
\hline
\end{tabular}

Table 12: Least Squares Means

\begin{tabular}{|l|c|c|c|c|c|c|c|c|c|}
\hline \multicolumn{1}{|l}{ Least Squares Means } \\
\hline Effect & Period & Estimate & $\begin{array}{c}\text { Standard } \\
\text { Error }\end{array}$ & df & t Value & Pr $>|\mathbf{t}|$ & Alpha & Lower & Upper \\
\hline Period & Baseline & 0.5686 & 0.01642 & 95 & 34.64 & $<.0001$ & 0.05 & 0.5360 & 0.6012 \\
\hline Period & 12-Month & 0.6626 & 0.03010 & 95 & 22.01 & $<.0001$ & 0.05 & 0.6028 & 0.7223 \\
\hline
\end{tabular}

\section{DISCUSSION}

Although FOCUS Butler County is funded as a service replication program, this study includes replication and extension of the original research. Questionnaires from the original FOCUS study (Boyer et al., 2005) were modified to pertain to our specific target population (females, 16-23, in community-based settings). Questions from the baseline, 4-month, and 12-month surveys were assigned to one, of ten, categories and each category's questions were compared for a proportion of change between baseline and 12-month responses. In this pilot, the only statistically significant changes were seen in the Knowledge-Based category, with a 9.4\% overall increase in correct responses. For questions that did not assess concrete, knowledge-based content, responses were coded with "agree" or "strongly agree" as affirmative, or "disagree" or "strongly disagree" as affirmative, based on the question, because of the Likert scale structure of the surveys.

One concept in the Knowledge-Based category, which showed a significant change, was participant understanding of pelvic inflammatory disease (PID). At baseline, only $50 \%$ of participants recognized the definition of PID, while at the 12-month follow-up, $85.71 \%$ were aware of the definition. A second concept in the KnowledgeBased category, with significant change, was the understanding that Implanon is an effective means of birth control. At baseline, a total of $33.69 \%$ identified Implanon as either "somewhat" or "very effective" at preventing pregnancy (both considered affirmative), and by the 12-month follow-up, a total of $75 \%$ of the respondents were aware that Implanon is effective (again, selecting "somewhat" or "very effective").

Although not statistically significant, there were a few other encouraging trends that were extracted from the data. For instance, at baseline, 45.26 believed that Depo-Provera is effective in preventing STDS and by the 12month follow-up, only $25 \%$ still believed this. Similarly, at baseline, $57.89 \%$ believed Implanon to be effective in preventing STDs. By the 12-month follow-up, only $21.43 \%$ of respondents had this misperception.

Because this study seeks to explore a phenomenon and because it is descriptive in nature, all raw data are presented in the following appendices: Appendix A - Knowledge-Based Questions; Appendix B - Reported Behaviors, Appendix C - Opinions; Appendix D - Perceived Opinions of Friends; Appendix E - Intentions for Future Practice; Appendix F - Perception of Risk; Appendix G - Attitudes Regarding Condom Use; Appendix H Attitudes Related to Alcohol Use; and Appendix I - Perception of Comfort Related to Health Seeking Behaviors. There were no statistically significant findings besides those previously noted in the knowledge-based category.

\section{LIMITATIONS}

Two key limitations noted in the pilot study include the homogenous sampling from one Ohio county and the small sample size, which may not be representative of the general population. The on-going research, for years two through five of the funded program, will incorporate much larger numbers - roughly 250-450 participants each 
year - and will include a four-county region rather than only one county. Other limitations of the study come with the descriptive design. The study is only meant to capture a "snapshot" of our target population's knowledge, beliefs, and behaviors. This is an essential first step in understanding the intricacies of teenage pregnancy for our target population. A final limitation that must be addressed is the fact that study participants conclude with a 12month post-intervention survey. A lot of living and learning happens in a 12-month period and it cannot be implied that changes in knowledge and/or behaviors are directly related to FOCUS participation, free of other influences.

\section{CONCLUSION}

The American College of Nurse Midwives (ACNM) recently released their Our Moment of Truth survey results (ACNM, 2013), which reported the following findings: 1) Women report that they don't feel knowledgeable about available types of birth control; 2) According to $40 \%$ of the women surveyed, health care providers don't properly inform women about how to correctly use their selected method of birth control; 3) Many women are having less than positive experiences when discussing birth control options with their healthcare providers; 4) Women have misperceptions about the effectiveness of birth control options; and 5) A number of women are not using the most effective forms of birth control available to them. The surveys were completed by 1,224 women between the ages of 18 and 45 years old. ACNM's timely report highlights potential contributing factors to the Center for Disease Control's estimates that more than one-third of pregnancies between 2006-2012 were unplanned (ACNM, 2013).

Next steps of this study include years 2-5 of the FOCUS Program, during the "full implementation" phase. Participants will include females from 16-19 years old, from a four county region. In order to reduce unplanned pregnancies in women of childbearing age, we must continue to explore their beliefs, behaviors, and barriers, which impact their outcomes. While teenage pregnancy rates, in particular, are not a new concern, each generation brings subsequent strengths and challenges. It is important that health care providers and researchers continue to build an accurate and current understanding of the" problem" in order to develop effective solutions.

\section{ACKNOWLEDGEMENTS}

This project was supported by Grant Number TP1AH000050-01-01 from the U.S. Department of Health and Human Services, Office of Adolescent Health. The content of this manuscript is solely the responsibility of the authors and does not necessarily represent the official views of the U.S. Department of Health and Human Services.

The authors would also like to acknowledge the YWCA Hamilton for its willingness to partner and the Miami University Undergraduate FOCUS team for endless enthusiasm, data entry, and table editing.

\section{AUTHOR INFORMATION}

Brooke Flinders received her associate's and bachelor's degrees in nursing from Miami University. She earned a certificate in nurse-midwifery (2005) and a master's degree in nursing (2006) from Frontier School of Midwifery and Family Nursing in Hyden, Kentucky. Her most recent clinical practice included full-scope midwifery services and in-hospital births. In 2010, Flinders partnered with the YWCA Hamilton to receive funding from the U.S. Department of Health and Human Services, as part of the Teen Pregnancy Prevention initiative. Her service-learning program educates teens on sexually transmitted infections and pregnancy prevention strategies. Her research explores teen attitudes and behaviors as well as the impacts of evidence-based programming. Flinders has incorporated hundreds of junior-level nursing students as "FOCUS" program educators and has worked with dozens of undergraduates, through her leadership and research internships. Flinders was recently promoted to Associate Professor and was granted tenure at Miami University.

Katelyn Gilb served as undergraduate Research Assistant for the FOCUS Program, during the pilot and first year of full implementation. She graduated from Miami University in May 2012, and is currently practicing as a registered nurse at a nearby county health department. In addition, she is the Program Assistant for the FOCUS Program. 
Tricia Neu served as an Undergraduate Associate for the FOCUS program during the fall and spring semesters of the 2012-2013 academic year. She currently is employed as a Research Assistant and will graduate from Miami University in May 2014.

\section{REFERENCES}

1. American College of Nurse-Midwives. (2013). Birth Control Methods: Most Popular by Use. American College of Nurse-Midwives.

2. American College of Nurse-Midwives. (2013). Family Planning \& Birth Control Survey Key Findings. Our Moment in Truth.

3. American College of Nurse-Midwives. (2010). New Survey Spotlights Women's Knowledge, Misperceptions About Birth Control Options and Effectiveness. Silver Spring, MD: American College of Nurse-Midwives.

4. $\quad$ American College of Nurse-Midwives. (2013, October 9). OMOT 2013 Survey Results. Retrieved from Our Moment of Truth: A new Understanding of Midwifery Care: http://www.midwife.org/OMOT-2013Survey-Results

5. American College of Nurse-Midwives. (2013). Our Moment of Truth 2013 Survey of Women's Heatlh Care Experiences and Perceptions: Spotlight on Family Planning and Contraception. Our Moment in Truth.

6. Avert. (2010, May 20). Averting HIV and AIDS. Retrieved February 28, 2014, from STDs in America: http://www.avert.org/stdstatisticusa.htm

7. Avert. (2014). Averting HIV and AIDS. Retrieved February 28, 2014, from STDs in America/AVERT: http://www.avert.org/stdstatisticusa.htm

8. Boyer, C. B., Shafer, M. A., Brodine, S. K., Pollack, L. M., Chang, Y. J., Kraft, H. S., et al. (2005). Evaluation of a cognitive-behavioral, group, randomized controlled intervention trial to prevent sexually transmitted infections and unintended pregnancies in young women. Preventive Medicine , 40, 420-431.

9. Card, J. J. (2001). The Sociometrics Program Archives: Promoting the dissemination of evidence-based practices through replication kits. Research on Social Work Practice , 11(4),521-526.

10. Card, J. J., Lessard, L., \& Benner, T. (2007). PASHA: Facilitating the replication and use of effective adolescent pregnancy and STI/HIV prevention programs. Journal of Adolescent Health, 40(3). 275 el-el14.

11. County Health Rankings \& Roadmaps: A Robert Wood Johnson Foundation Programunder the direction of the University of Wisconsion Population Health Institue. Retrieved February 28, 2014, from: http://www.countyhealthrankings.org/ohio/erie/14

12. County Health Rankings \& Roadmaps: A Robert Wood Johnson Foundation Programunder the direction of the University of Wisconsion Population Health Institue. Retrieved May 21, 2010, from Table 1: Butler County Health Statistics (Data Collected 2000-2006): http://www.countyhealthrankings.org/ohio/butler

13. County Health Rankings and Roadmaps: A Robert Wood Johnson Foundation Program under the direction of the University of Wisconsin Population Health Institute. Retrieved May 21, 2010 from: http://www.countyhealthrankings.org/ohio/erie/14

14. County Health Rankings and Roadmaps: A Robert Wood Johnson Foundation Program under the direction of the University of Wisconsin Population Health Institute. Retrieved May 21, 2010 from http://www.countyhealthrankings.org/ohio/butler. (n.d.). Retrieved May 21, 2010, from countyhealthrankings.org: http://www.countyhealthrankings.org/ohio/butler

15. Our Moment Of Truth. (2013, October 9). OMOT 2013 Survey Results. Retrieved from American College of Nurse- MidWives: http://www.midwife.org/OMOT-2013-Survey-Results

16. Science-based Products for Researchers and Practitioners. (2011, September 11). Sociometrics Teen Pregnancy Prevention Programs . Retrieved 2011, from Http://www.socio.com/pashatable.php.

17. U.S Department of Health \& Human Services (2013, Decemeber 12). Office Of adolescent Health . Retrieved February 26, 2014, from hhs.gov: http://www.hhs.cov/ash/oah/about-us/index.html

18. Sociometrics. (2005). Focus: Preventing sexually transmitted infections and unintended pregnancies among young women. Los, Altos, CA: Program Archive on Sexuality, Health, and Adolescence (PASHA).

19. Sociometrics; U.S. Department of Health and Human Services. (2011, September 11). Teen Pregnancy. Retrieved 2011, from Science-based products for researchers \& practitioners: http://www.socio.com/pashatable.php 
20. U.S. Department of Health \& Human Services. (2009). Community Health Status Indicators. Retrieved February 28, 2014, from CHSI 2009:

(http://www.communityhealth.hhs.gov/Demographics.aspx?GeogCD=39017\&PeerStrat=9\&state=Ohio\&co unty=Butler

21. U.S. Department of Health \& Human Services. (2010, May 20). Community Health Status Indicators 2009. Retrieved February 28, 2014, from Table 2 Health Statistics for Bulter County as Compared to "Peer" Counties:

(http://www.communityhealth.hhs.gov/Demographics.aspx?GeogCD=39017\&PeerStrat=9\&state=Ohio\&co unty=Butler

22. U.S. Department of Health \& Human Services. (2013, Decemeber 12). Office Of adolescent Health. Retrieved February 26, 2014, from Adolescent Health Topics;OAH initiatives: http://www.hhs.cov/ash/oah/about-us/index.html

23. U.S. Department of Health and Human Services . (2013, August 28). HealthyPeaople.gov. Retrieved May 17, 2010, from Healthy People 2020-Improving the Health of Americans: http://www.healthypeople.gov/hp2020/objectives/TopicArea.aspx?id=21\&TopicArea=Family+Planning

24. U.S. Department of Health and Human Services. (2010, May 17). Data on Condom Use. Retrieved May 17 , 2010, from Healthy People 2020-Improving the Health of Americans:

http://www.healthypeople.gov/hp2020/objectives/TopicArea.aspx?id=21\&TopicArea=Family+Planning 


\section{APPENDIX A: KNOWLEDGE-BASED QUESTIONS}

\begin{tabular}{|c|c|c|c|c|c|c|}
\hline \multicolumn{7}{|c|}{ Knowledge-Based Question } \\
\hline \multirow{2}{*}{ Survey Period } & \multicolumn{6}{|c|}{ Safe-Sex Includes Activities Such As Hugging/Body Massages/Slow Dancing. } \\
\hline & Strongly Agree & $\begin{array}{c}\text { Agree } \\
\text { Somewhat }\end{array}$ & Neutral & $\begin{array}{l}\text { Disagree } \\
\text { Somewhat }\end{array}$ & $\begin{array}{l}\text { Strongly } \\
\text { Disagree }\end{array}$ & Tota \\
\hline Baseline & $\begin{array}{c}15 \\
15.79 \%\end{array}$ & $\begin{array}{c}10 \\
10.53 \%\end{array}$ & $\begin{array}{c}25 \\
26.32 \%\end{array}$ & $\begin{array}{c}10 \\
10.53 \%\end{array}$ & $\begin{array}{c}35 \\
36.84 \%\end{array}$ & 95 \\
\hline 12-Month & $\begin{array}{c}11 \\
39.29 \%\end{array}$ & $\begin{array}{c}2 \\
7.14 \%\end{array}$ & $\begin{array}{c}4 \\
14.29 \%\end{array}$ & $\begin{array}{c}1 \\
3.57 \%\end{array}$ & $\begin{array}{c}10 \\
35.7 \% 1\end{array}$ & 28 \\
\hline Total \# & 26 & 12 & 29 & 11 & 45 & 123 \\
\hline \multicolumn{7}{|c|}{ Frequency Missing = 1} \\
\hline
\end{tabular}

\begin{tabular}{|c|c|c|c|c|c|c|}
\hline \multicolumn{7}{|c|}{ Knowledge-Based Question } \\
\hline \multirow[t]{2}{*}{ Survey Period } & \multicolumn{6}{|c|}{ Getting An STD Increases The Risk Of Getting HIV. } \\
\hline & Strongly Agree & \begin{tabular}{|l|} 
Agree \\
Somewhat
\end{tabular} & Neutral & \begin{tabular}{|l} 
Disagree \\
Somewhat
\end{tabular} & $\begin{array}{l}\text { Strongly } \\
\text { Disagree }\end{array}$ & Total \\
\hline Baseline & $\begin{array}{c}24 \\
25.00 \%\end{array}$ & $\begin{array}{c}33 \\
34.38 \%\end{array}$ & $\begin{array}{c}22 \\
22.92 \%\end{array}$ & $\begin{array}{c}8 \\
8.33 \%\end{array}$ & $\begin{array}{c}9 \\
9.38 \%\end{array}$ & 96 \\
\hline 12-Month & $\begin{array}{c}6 \\
21.43 \% \\
\end{array}$ & $\begin{array}{c}13 \\
46.43 \%\end{array}$ & $\begin{array}{c}5 \\
17.86 \%\end{array}$ & $\begin{array}{c}2 \\
7.14 \%\end{array}$ & $\begin{array}{c}2 \\
7.14 \%\end{array}$ & 28 \\
\hline Total \# & 30 & 46 & 27 & 10 & 11 & 124 \\
\hline
\end{tabular}

\begin{tabular}{|c|c|c|c|c|c|c|}
\hline \multicolumn{7}{|c|}{ Knowledge-Based Question } \\
\hline \multirow[t]{2}{*}{ Survey Period } & \multicolumn{6}{|c|}{$\begin{array}{l}\text { Condoms Called "Natural Skins" Are Not Recommended Because They Don't Prevent HIV } \\
\text { And Other STDs. }\end{array}$} \\
\hline & Strongly Agree & $\begin{array}{c}\text { Agree } \\
\text { Somewhat }\end{array}$ & Neutral & $\begin{array}{l}\text { Disagree } \\
\text { Somewhat }\end{array}$ & $\begin{array}{l}\text { Strongly } \\
\text { Disagree }\end{array}$ & Total \\
\hline Baseline & $\begin{array}{c}10 \\
10.53 \%\end{array}$ & $\begin{array}{c}18 \\
18.95 \%\end{array}$ & $\begin{array}{c}28 \\
29.47 \%\end{array}$ & $\begin{array}{c}15 \\
15.79 \%\end{array}$ & $\begin{array}{c}24 \\
25.26 \%\end{array}$ & 95 \\
\hline 12-Month & $\begin{array}{c}2 \\
7.14 \%\end{array}$ & $\begin{array}{c}2 \\
7.14 \%\end{array}$ & $\begin{array}{c}7 \\
25.00 \% \\
\end{array}$ & $\begin{array}{c}5 \\
17.86 \%\end{array}$ & $\begin{array}{c}12 \\
42.86 \%\end{array}$ & 28 \\
\hline Total \# & 12 & 20 & 35 & 20 & 36 & 123 \\
\hline
\end{tabular}

\begin{tabular}{|c|c|c|c|c|c|c|}
\hline \multicolumn{7}{|c|}{ Knowledge-Based Question } \\
\hline \multirow[t]{2}{*}{ Survey Period } & \multicolumn{6}{|c|}{ With Safer-Sexual Activities, There Is The Possibility That Fluids Can Be Exchanged. } \\
\hline & Strongly Agree & $\begin{array}{c}\text { Agree } \\
\text { Somewhat }\end{array}$ & Neutral & $\begin{array}{c}\text { Disagree } \\
\text { Somewhat }\end{array}$ & $\begin{array}{l}\text { Strongly } \\
\text { Disagree }\end{array}$ & Total \\
\hline Baseline & $\begin{array}{c}38 \\
39.58 \% \\
\end{array}$ & $\begin{array}{c}30 \\
31.25 \%\end{array}$ & $\begin{array}{c}9 \\
9.38 \%\end{array}$ & $\begin{array}{c}9 \\
9.38 \%\end{array}$ & $\begin{array}{c}10 \\
10.42 \%\end{array}$ & 96 \\
\hline 12-Month & $\begin{array}{c}14 \\
50.00 \%\end{array}$ & $\begin{array}{c}6 \\
21.43 \% \\
\end{array}$ & $\begin{array}{c}5 \\
17.86 \% \\
\end{array}$ & $\begin{array}{c}1 \\
3.57 \%\end{array}$ & $\begin{array}{c}2 \\
7.14 \%\end{array}$ & 28 \\
\hline Total \# & 52 & 36 & 14 & 10 & 12 & 124 \\
\hline
\end{tabular}

\begin{tabular}{|c|c|c|c|c|c|c|}
\hline \multicolumn{7}{|c|}{ Knowledge-Based Question } \\
\hline \multirow[t]{2}{*}{ Survey Period } & \multicolumn{6}{|c|}{ Some STDs Can Be Transmitted By Exposure To Open Sores When Kissing. } \\
\hline & Strongly Agree & $\begin{array}{c}\text { Agree } \\
\text { Somewhat }\end{array}$ & Neutral & $\begin{array}{l}\text { Disagree } \\
\text { Somewhat }\end{array}$ & $\begin{array}{l}\text { Strongly } \\
\text { Disagree }\end{array}$ & Total \\
\hline Baseline & $\begin{array}{c}58 \\
61.05 \%\end{array}$ & $\begin{array}{c}27 \\
28.42 \%\end{array}$ & $\begin{array}{c}5 \\
5.26 \%\end{array}$ & $\begin{array}{c}3 \\
3.16 \%\end{array}$ & $\begin{array}{c}2 \\
2.11 \%\end{array}$ & 95 \\
\hline 12-Month & $\begin{array}{c}15 \\
53.57 \%\end{array}$ & $\begin{array}{c}9 \\
32.14 \%\end{array}$ & $\begin{array}{c}1 \\
3.57 \%\end{array}$ & $\begin{array}{c}0 \\
0.00 \%\end{array}$ & $\begin{array}{c}3 \\
10.71 \%\end{array}$ & 28 \\
\hline Total \# & 73 & 36 & 6 & 3 & 5 & 123 \\
\hline
\end{tabular}




\begin{tabular}{|c|c|c|c|c|c|c|}
\hline \multicolumn{7}{|c|}{ Knowledge-Based Question } \\
\hline \multirow[t]{2}{*}{ Survey Period } & \multicolumn{6}{|c|}{ STDs Can Be Transmitted Through Ear Piercing, If Sterile Needles Are Not Used. } \\
\hline & Strongly Agree & $\begin{array}{c}\text { Agree } \\
\text { Somewhat }\end{array}$ & Neutral & $\begin{array}{c}\text { Disagree } \\
\text { Somewhat }\end{array}$ & $\begin{array}{l}\text { Strongly } \\
\text { Disagree }\end{array}$ & Total \\
\hline Baseline & $\begin{array}{c}58 \\
60.42 \% \\
\end{array}$ & $\begin{array}{c}23 \\
23.96 \%\end{array}$ & $\begin{array}{c}5 \\
5.21 \%\end{array}$ & $\begin{array}{c}3 \\
3.13 \%\end{array}$ & $\begin{array}{c}7 \\
7.29 \%\end{array}$ & 96 \\
\hline 12-Month & $\begin{array}{c}18 \\
66.67 \%\end{array}$ & $\begin{array}{c}6 \\
22.22 \% \\
\end{array}$ & $\begin{array}{c}1 \\
3.70 \%\end{array}$ & $\begin{array}{c}0 \\
0.00 \%\end{array}$ & $\begin{array}{c}2 \\
7.41 \%\end{array}$ & 27 \\
\hline Total \# & 76 & 29 & 6 & 3 & 9 & 123 \\
\hline
\end{tabular}

\begin{tabular}{|c|c|c|c|c|c|c|}
\hline \multicolumn{7}{|c|}{ Knowledge-Based Question } \\
\hline \multirow[t]{2}{*}{ Survey Period } & \multicolumn{6}{|c|}{$\begin{array}{l}\text { The Vagina/Cervix Is Covered By Thin Cells And Makes Women More Susceptible To Be } \\
\text { Infected. }\end{array}$} \\
\hline & Strongly Agree & $\begin{array}{c}\text { Agree } \\
\text { Somewhat }\end{array}$ & Neutral & $\begin{array}{c}\text { Disagree } \\
\text { Somewhat }\end{array}$ & $\begin{array}{l}\text { Strongly } \\
\text { Disagree }\end{array}$ & Total \\
\hline Baseline & $\begin{array}{c}23 \\
24.47 \%\end{array}$ & $\begin{array}{c}25 \\
26.60 \%\end{array}$ & $\begin{array}{c}34 \\
36.17 \%\end{array}$ & $\begin{array}{c}5 \\
5.32 \%\end{array}$ & $\begin{array}{c}7 \\
7.45 \%\end{array}$ & 94 \\
\hline 12-Month & $\begin{array}{c}12 \\
42.86 \%\end{array}$ & $\begin{array}{c}8 \\
28.57 \%\end{array}$ & $\begin{array}{c}5 \\
17.86 \%\end{array}$ & $\begin{array}{c}3 \\
10.71 \%\end{array}$ & $\begin{array}{c}0 \\
0.00 \%\end{array}$ & 28 \\
\hline Total \# & 35 & 33 & 39 & 8 & 7 & 122 \\
\hline
\end{tabular}

\begin{tabular}{|c|c|c|c|c|c|c|}
\hline \multicolumn{7}{|c|}{ Knowledge-Based Question } \\
\hline \multirow[t]{2}{*}{ Survey Period } & \multicolumn{6}{|c|}{ Using Condoms Gives A Woman Complete Protection Against STDs. } \\
\hline & Strongly Agree & $\begin{array}{c}\text { Agree } \\
\text { Somewhat }\end{array}$ & Neutral & $\begin{array}{c}\text { Disagree } \\
\text { Somewhat }\end{array}$ & $\begin{array}{l}\text { Strongly } \\
\text { Disagree }\end{array}$ & Total \\
\hline Baseline & $\begin{array}{c}4 \\
4.26 \% \\
\end{array}$ & $\begin{array}{c}13 \\
13.83 \%\end{array}$ & $\begin{array}{c}7 \\
7.45 \%\end{array}$ & $\begin{array}{c}22 \\
23.40 \%\end{array}$ & $\begin{array}{c}48 \\
51.06 \%\end{array}$ & 94 \\
\hline 12-month & $\begin{array}{c}1 \\
3.57 \% \\
\end{array}$ & $\begin{array}{c}1 \\
3.57 \% \\
\end{array}$ & $\begin{array}{c}3 \\
10.71 \% \\
\end{array}$ & $\begin{array}{c}4 \\
14.29 \% \\
\end{array}$ & $\begin{array}{c}19 \\
67.86 \% \\
\end{array}$ & 28 \\
\hline Total \# & 5 & 14 & 10 & 26 & 67 & 122 \\
\hline
\end{tabular}

\begin{tabular}{|l|c|c|c|c|c|c|}
\hline Knowledge-Based Question \\
\hline Survey Period & HIV Can Be Transmitted Through 'Dry Kissing'. & \multicolumn{1}{l|}{} \\
\hline & Strongly Agree & $\begin{array}{c}\text { Agree } \\
\text { Somewhat }\end{array}$ & Neutral & $\begin{array}{c}\text { Disagree } \\
\text { Somewhat }\end{array}$ & $\begin{array}{c}\text { Strongly } \\
\text { Disagree }\end{array}$ & Total \\
\hline Baseline & 3 & 8 & 19 & 21 & 45 & \\
\hline \multirow{2}{*}{ 12-Month } & $3.13 \%$ & $8.33 \%$ & $19.79 \%$ & $21.88 \%$ & $46.88 \%$ & 96 \\
\hline Total \# & 4 & 2 & 4 & 9 & 9 & 28 \\
\hline
\end{tabular}

\begin{tabular}{|c|c|c|c|c|c|c|}
\hline \multicolumn{7}{|c|}{ Knowledge-Based Question } \\
\hline \multirow[t]{2}{*}{ Survey Period } & \multicolumn{6}{|c|}{ Condoms Used With Lubricants Is The Best Method For Preventing STDs. } \\
\hline & Strongly Agree & $\begin{array}{c}\text { Agree } \\
\text { Somewhat }\end{array}$ & Neutral & $\begin{array}{c}\text { Disagree } \\
\text { Somewhat }\end{array}$ & $\begin{array}{l}\text { Strongly } \\
\text { Disagree }\end{array}$ & Total \\
\hline Baseline & $\begin{array}{c}2 \\
2.08 \%\end{array}$ & $\begin{array}{c}7 \\
7.29 \%\end{array}$ & $\begin{array}{c}28 \\
29.17 \%\end{array}$ & $\begin{array}{c}19 \\
19.79 \%\end{array}$ & $\begin{array}{c}40 \\
41.67 \%\end{array}$ & 96 \\
\hline 12-Month & $\begin{array}{c}2 \\
7.14 \%\end{array}$ & $\begin{array}{c}2 \\
7.14 \%\end{array}$ & $\begin{array}{c}6 \\
21.43 \% \\
\end{array}$ & $\begin{array}{c}5 \\
17.86 \%\end{array}$ & $\begin{array}{c}13 \\
46.43 \% \\
\end{array}$ & 28 \\
\hline Total \# & 4 & 9 & 34 & 24 & 53 & 124 \\
\hline
\end{tabular}




\begin{tabular}{|l|c|c|c|c|c|c|}
\hline Knowledge-Based Question \\
\hline Survey Period & Women With HIV Can Give It To Their Babies Through Their Breast Milk. \\
\hline & Strongly Agree & $\begin{array}{c}\text { Agree } \\
\text { Somewhat }\end{array}$ & Neutral & $\begin{array}{c}\text { Disagree } \\
\text { Somewhat }\end{array}$ & $\begin{array}{c}\text { Strongly } \\
\text { Disagree }\end{array}$ & Total \\
\hline Baseline & 34 & 29 & 24 & 4 & 5 & \\
\hline \multirow{2}{*}{ 12-Month } & $35.42 \%$ & $30.21 \%$ & $25.00 \%$ & $4.17 \%$ & $5.21 \%$ & 96 \\
\hline Total \# & 16 & 3 & 4 & 2 & 3 & 28 \\
\hline
\end{tabular}

\begin{tabular}{|c|c|c|c|c|c|c|}
\hline \multicolumn{7}{|c|}{ Knowledge-Based Question } \\
\hline \multirow{2}{*}{ Survey Period } & \multicolumn{6}{|c|}{ Withdrawal Of The Penis Before Ejaculation Prevents STDs. } \\
\hline & Strongly Agree & $\begin{array}{c}\text { Agree } \\
\text { Somewhat }\end{array}$ & Neutral & $\begin{array}{c}\text { Disagree } \\
\text { Somewhat }\end{array}$ & $\begin{array}{l}\text { Strongly } \\
\text { Disagree }\end{array}$ & Total \\
\hline Baseline & $\begin{array}{c}3 \\
3.13 \% \\
\end{array}$ & $\begin{array}{c}6 \\
6.25 \%\end{array}$ & $\begin{array}{c}14 \\
14.58 \%\end{array}$ & $\begin{array}{c}13 \\
13.54 \%\end{array}$ & $\begin{array}{c}60 \\
62.50 \%\end{array}$ & 96 \\
\hline 12-Month & $\begin{array}{c}3 \\
10.71 \%\end{array}$ & $\begin{array}{c}1 \\
3.57 \%\end{array}$ & $\begin{array}{c}1 \\
3.57 \%\end{array}$ & $\begin{array}{c}0 \\
0.00 \%\end{array}$ & $\begin{array}{c}23 \\
82.14 \%\end{array}$ & 28 \\
\hline Total \# & 6 & 7 & 15 & 13 & 83 & 124 \\
\hline
\end{tabular}

\begin{tabular}{|l|c|c|c|c|c|c|}
\hline Knowledge-Based Question \\
\hline Survey Period & Having An HIV Test Done 2 Weeks After Sex Can Tell Whether Or Not You Have HIV. \\
\hline & Strongly Agree & $\begin{array}{c}\text { Agree } \\
\text { Somewhat }\end{array}$ & Neutral & $\begin{array}{c}\text { Disagree } \\
\text { Somewhat }\end{array}$ & $\begin{array}{c}\text { Strongly } \\
\text { Disagree }\end{array}$ & Total \\
\hline Baseline & 15 & 12 & 38 & 13 & 18 & \\
& $15.63 \%$ & $12.50 \%$ & $39.58 \%$ & $13.54 \%$ & $18.75 \%$ & 96 \\
\hline \multirow{2}{*}{ 12-Month } & 3 & 2 & 8 & 7 & 8 & 28 \\
\hline Total \# & $10.71 \%$ & $7.14 \%$ & $28.57 \%$ & $25.00 \%$ & $28.57 \%$ & 26 \\
\hline
\end{tabular}

\begin{tabular}{|c|c|c|c|c|c|c|}
\hline \multicolumn{7}{|c|}{ Knowledge-Based Question } \\
\hline \multirow[t]{2}{*}{ Survey Period } & \multicolumn{6}{|c|}{ STDs Can Be Passed From Woman To Baby During Birth, Even If She Has No Symptoms. } \\
\hline & Strongly Agree & $\begin{array}{c}\text { Agree } \\
\text { Somewhat }\end{array}$ & Neutral & $\begin{array}{l}\text { Disagree } \\
\text { Somewhat }\end{array}$ & $\begin{array}{c}\text { Strongly } \\
\text { Disagree }\end{array}$ & Total \\
\hline Baseline & $\begin{array}{c}44 \\
45.83 \%\end{array}$ & $\begin{array}{c}27 \\
28.13 \%\end{array}$ & $\begin{array}{c}17 \\
17.71 \%\end{array}$ & $\begin{array}{c}2 \\
2.08 \%\end{array}$ & $\begin{array}{c}6 \\
6.25 \% \\
\end{array}$ & 96 \\
\hline 12-Month & $\begin{array}{c}17 \\
60.71 \%\end{array}$ & $\begin{array}{c}7 \\
25.00 \%\end{array}$ & $\begin{array}{c}3 \\
10.71 \%\end{array}$ & $\begin{array}{c}1 \\
3.57 \%\end{array}$ & $\begin{array}{c}0 \\
0.00 \%\end{array}$ & 28 \\
\hline Total \# & 61 & 34 & 20 & 3 & 6 & 124 \\
\hline
\end{tabular}

\begin{tabular}{|c|c|c|c|c|c|}
\hline \multicolumn{6}{|c|}{ Knowledge-Based Question } \\
\hline \multirow[t]{2}{*}{ Survey Period } & \multicolumn{5}{|c|}{ A Person Can Be Infected With HIV For Five/More Years Without Developing AIDS. } \\
\hline & Strongly Agree & $\begin{array}{c}\text { Agree } \\
\text { Somewhat }\end{array}$ & Neutral & $\begin{array}{l}\text { Strongly } \\
\text { Disagree }\end{array}$ & Total \\
\hline Baseline & $\begin{array}{c}33 \\
34.38 \% \\
\end{array}$ & $\begin{array}{c}25 \\
26.04 \% \\
\end{array}$ & $\begin{array}{c}33 \\
34.38 \% \\
\end{array}$ & $\begin{array}{c}5 \\
5.21 \% \\
\end{array}$ & 96 \\
\hline 12-Month & $\begin{array}{c}18 \\
64.29 \% \\
\end{array}$ & $\begin{array}{c}5 \\
17.86 \% \\
\end{array}$ & $\begin{array}{c}4 \\
14.29 \% \\
\end{array}$ & $\begin{array}{c}1 \\
3.57 \%\end{array}$ & 28 \\
\hline Total \# & 51 & 30 & 37 & 6 & 124 \\
\hline
\end{tabular}




\begin{tabular}{|c|c|c|c|c|c|c|}
\hline \multicolumn{7}{|c|}{ Knowledge-Based Question } \\
\hline \multirow[t]{2}{*}{ Survey Period } & \multicolumn{6}{|c|}{ A Woman With An STD Always Has Symptoms. } \\
\hline & Strongly Agree & $\begin{array}{c}\text { Agree } \\
\text { Somewhat }\end{array}$ & Neutral & $\begin{array}{l}\text { Disagree } \\
\text { Somewhat }\end{array}$ & $\begin{array}{l}\text { Strongly } \\
\text { Disagree }\end{array}$ & Total \\
\hline Baseline & $\begin{array}{c}4 \\
4.21 \% \\
\end{array}$ & $\begin{array}{c}7 \\
7.37 \%\end{array}$ & $\begin{array}{c}16 \\
16.84 \%\end{array}$ & $\begin{array}{c}13 \\
13.68 \%\end{array}$ & $\begin{array}{c}55 \\
57.89 \%\end{array}$ & 95 \\
\hline 12-Month & $\begin{array}{c}2 \\
7.14 \%\end{array}$ & $\begin{array}{c}2 \\
7.14 \%\end{array}$ & $\begin{array}{c}1 \\
3.57 \%\end{array}$ & $\begin{array}{c}4 \\
14.29 \%\end{array}$ & $\begin{array}{c}19 \\
67.86 \%\end{array}$ & 28 \\
\hline Total \# & 6 & 9 & 17 & 17 & 74 & 123 \\
\hline
\end{tabular}

\begin{tabular}{|c|c|c|c|c|c|c|}
\hline \multicolumn{7}{|c|}{ Knowledge-Based Question } \\
\hline \multirow[t]{2}{*}{ Survey Period } & \multicolumn{6}{|c|}{ The Human Papillomavirus (Warts) Can Lead To Cervical Cancer. } \\
\hline & Strongly Agree & $\begin{array}{c}\text { Agree } \\
\text { Somewhat }\end{array}$ & Neutral & $\begin{array}{c}\text { Disagree } \\
\text { Somewhat }\end{array}$ & $\begin{array}{l}\text { Strongly } \\
\text { Disagree }\end{array}$ & Total \\
\hline Baseline & $\begin{array}{c}38 \\
40.00 \%\end{array}$ & $\begin{array}{c}23 \\
24.21 \%\end{array}$ & $\begin{array}{c}31 \\
32.63 \% \\
\end{array}$ & $\begin{array}{c}1 \\
1.05 \%\end{array}$ & $\begin{array}{c}2 \\
2.11 \%\end{array}$ & 95 \\
\hline 12-Month & $\begin{array}{c}20 \\
71.43 \%\end{array}$ & $\begin{array}{c}2 \\
7.14 \%\end{array}$ & $\begin{array}{c}5 \\
17.86 \%\end{array}$ & $\begin{array}{c}0 \\
0.00 \%\end{array}$ & $\begin{array}{c}1 \\
3.57 \% \\
\end{array}$ & 28 \\
\hline Total \# & 58 & 25 & 36 & 1 & 3 & 123 \\
\hline \multicolumn{7}{|c|}{ Frequency Missing = 1} \\
\hline
\end{tabular}

\begin{tabular}{|c|c|c|c|c|c|c|}
\hline \multicolumn{7}{|c|}{ Knowledge-Based Question } \\
\hline \multirow[t]{2}{*}{ Survey Period } & \multicolumn{6}{|c|}{$\begin{array}{l}\text { If Woman's Tubes Are Blocked From Scars, Her Eggs Can Get Stuck Causing An Ectopic } \\
\text { Pregnancy. }\end{array}$} \\
\hline & Strongly Agree & $\begin{array}{c}\text { Agree } \\
\text { Somewhat }\end{array}$ & Neutral & $\begin{array}{l}\text { Disagree } \\
\text { Somewhat }\end{array}$ & $\begin{array}{l}\text { Strongly } \\
\text { Disagree }\end{array}$ & Total \\
\hline Baseline & $\begin{array}{c}27 \\
28.13 \%\end{array}$ & $\begin{array}{c}26 \\
27.08 \%\end{array}$ & $\begin{array}{c}39 \\
40.63 \%\end{array}$ & $\begin{array}{c}3 \\
3.13 \%\end{array}$ & $\begin{array}{c}1 \\
1.04 \%\end{array}$ & 96 \\
\hline 12-Month & $\begin{array}{c}17 \\
60.71 \%\end{array}$ & $\begin{array}{c}7 \\
25.00 \%\end{array}$ & $\begin{array}{c}2 \\
7.14 \%\end{array}$ & $\begin{array}{c}2 \\
7.14 \%\end{array}$ & $0.00 \%$ & 28 \\
\hline Total \# & 44 & 33 & 41 & 5 & 1 & 124 \\
\hline
\end{tabular}

\begin{tabular}{|c|c|c|c|c|c|c|}
\hline \multicolumn{7}{|c|}{ Knowledge-Based Question } \\
\hline \multirow[t]{2}{*}{ Survey Period } & \multicolumn{6}{|c|}{ The Female Condom Is A Pouch With Internal And External Rings. } \\
\hline & Strongly Agree & $\begin{array}{c}\text { Agree } \\
\text { Somewhat }\end{array}$ & Neutral & $\begin{array}{c}\text { Disagree } \\
\text { Somewhat }\end{array}$ & $\begin{array}{l}\text { Strongly } \\
\text { Disagree }\end{array}$ & Total \\
\hline Baseline & $\begin{array}{c}29 \\
30.53 \% \\
\end{array}$ & $\begin{array}{c}28 \\
29.47 \%\end{array}$ & $\begin{array}{c}32 \\
33.68 \%\end{array}$ & $\begin{array}{c}5 \\
5.26 \%\end{array}$ & $\begin{array}{c}1 \\
1.05 \%\end{array}$ & 95 \\
\hline 12-Month & $\begin{array}{c}14 \\
51.85 \%\end{array}$ & $\begin{array}{c}9 \\
33.33 \% \\
\end{array}$ & $\begin{array}{c}2 \\
7.41 \% \\
\end{array}$ & $\begin{array}{c}2 \\
7.41 \% \\
\end{array}$ & $\begin{array}{c}0 \\
0.00 \% \\
\end{array}$ & 27 \\
\hline Total \# & 43 & 37 & 34 & 7 & 1 & 122 \\
\hline
\end{tabular}

\begin{tabular}{|l|c|c|c|c|c|}
\hline Knowledge-Based Question & \multicolumn{6}{l|}{} \\
\hline Survey Period & Women With Particular STDs May Have Rashes/Lumps Around Vagina/Anus/Mouth. \\
& Strongly Agree & $\begin{array}{c}\text { Agree } \\
\text { Somewhat }\end{array}$ & Neutral & Strongly Disagree & Total \\
\hline Baseline & 49 & 30 & 15 & 2 & 96 \\
& $51.04 \%$ & $31.25 \%$ & $15.63 \%$ & $2.08 \%$ & 28 \\
\hline 12-Month & 21 & 5 & 1 & $3.57 \%$ & 124 \\
\hline Total \# & $75.00 \%$ & $17.86 \%$ & $3.57 \%$ & 3 & \multirow{2}{*}{16} \\
\hline
\end{tabular}




\begin{tabular}{|l|c|c|c|c|c|}
\hline Knowledge-Based Question & \multicolumn{5}{|c|}{} \\
\hline Survey Period & PID Is An Infection Of The Uterus, Ovaries, And Fallopian Tubes. & $\begin{array}{c}\text { Strongly } \\
\text { Disagree }\end{array}$ & Total \\
\hline & Strongly Agree & $\begin{array}{c}\text { Agree } \\
\text { Somewhat }\end{array}$ & Neutral & 2 & 94 \\
Baseline & 19 & 28 & 45 & $2.13 \%$ & 2 \\
\hline 12-Month & $20.21 \%$ & $29.79 \%$ & $47.87 \%$ & $7.14 \%$ & 28 \\
\hline Total \# & 14 & 10 & $7.14 \%$ & 4 & 122 \\
\hline Frequency Missing = & $50.00 \%$ & $35.71 \%$ & 47 & & \\
\hline
\end{tabular}

\begin{tabular}{|c|c|c|c|c|c|c|}
\hline \multicolumn{7}{|c|}{ Knowledge-Based Question } \\
\hline \multirow[t]{2}{*}{ Survey Period } & \multicolumn{6}{|c|}{$\begin{array}{l}\text { Viral STDs Can Be Treated To Make Some Of The Symptoms Improve, But There Are No } \\
\text { Cures. }\end{array}$} \\
\hline & Strongly Agree & $\begin{array}{c}\text { Agree } \\
\text { Somewhat }\end{array}$ & Neutral & $\begin{array}{c}\text { Disagree } \\
\text { Somewhat }\end{array}$ & $\begin{array}{l}\text { Strongly } \\
\text { Disagree }\end{array}$ & Total \\
\hline Baseline & $\begin{array}{c}38 \\
39.58 \% \\
\end{array}$ & $\begin{array}{c}20 \\
20.83 \%\end{array}$ & $\begin{array}{c}27 \\
28.13 \%\end{array}$ & $\begin{array}{c}7 \\
7.29 \%\end{array}$ & $\begin{array}{c}4 \\
4.17 \%\end{array}$ & 96 \\
\hline 12-Month & $\begin{array}{c}21 \\
75.00 \%\end{array}$ & $\begin{array}{c}3 \\
10.71 \%\end{array}$ & $\begin{array}{c}1 \\
3.57 \%\end{array}$ & $\begin{array}{c}2 \\
7.14 \%\end{array}$ & $\begin{array}{c}1 \\
3.57 \%\end{array}$ & 28 \\
\hline Total \# & 59 & 23 & 28 & 9 & 5 & 124 \\
\hline
\end{tabular}

\begin{tabular}{|c|c|c|c|c|c|c|}
\hline \multicolumn{7}{|c|}{ Knowledge-Based Question } \\
\hline \multirow[t]{2}{*}{ Survey Period } & \multicolumn{6}{|c|}{ If A Woman Has Scarring/Blockage In Her Tubes, She May Become Sterile. } \\
\hline & Strongly Agree & $\begin{array}{c}\text { Agree } \\
\text { Somewhat }\end{array}$ & Neutral & $\begin{array}{c}\text { Disagree } \\
\text { Somewhat }\end{array}$ & $\begin{array}{l}\text { Strongly } \\
\text { Disagree }\end{array}$ & Total \\
\hline Baseline & $\begin{array}{c}33 \\
34.74 \%\end{array}$ & $\begin{array}{c}21 \\
22.11 \%\end{array}$ & $\begin{array}{c}37 \\
38.95 \%\end{array}$ & $\begin{array}{c}2 \\
2.11 \%\end{array}$ & $\begin{array}{c}2 \\
2.11 \%\end{array}$ & 95 \\
\hline 12-Month & $\begin{array}{c}15 \\
53.57 \%\end{array}$ & $\begin{array}{c}8 \\
28.57 \%\end{array}$ & $\begin{array}{c}3 \\
10.71 \%\end{array}$ & $\begin{array}{c}1 \\
3.57 \%\end{array}$ & $\begin{array}{c}1 \\
3.57 \%\end{array}$ & 28 \\
\hline Total \# & 48 & 29 & 40 & 3 & 3 & 123 \\
\hline \multicolumn{7}{|c|}{ Frequency Missing = 1} \\
\hline
\end{tabular}

\begin{tabular}{|c|c|c|c|c|c|c|}
\hline \multicolumn{7}{|c|}{ Knowledge-Based Question } \\
\hline \multirow[t]{2}{*}{ Survey Period } & \multicolumn{6}{|c|}{ Some STDs Can Harm The Brains Of Unborn Babies, Causing Mental Retardation. } \\
\hline & Strongly Agree & $\begin{array}{c}\text { Agree } \\
\text { Somewhat }\end{array}$ & Neutral & $\begin{array}{l}\text { Disagree } \\
\text { Somewhat }\end{array}$ & $\begin{array}{l}\text { Strongly } \\
\text { Disagree }\end{array}$ & Total \\
\hline Baseline & $\begin{array}{c}22 \\
23.40 \%\end{array}$ & $\begin{array}{c}27 \\
28.72 \%\end{array}$ & $\begin{array}{c}32 \\
34.04 \%\end{array}$ & $\begin{array}{c}11 \\
11.70 \%\end{array}$ & $\begin{array}{c}2 \\
2.13 \%\end{array}$ & 94 \\
\hline 12-Month & $\begin{array}{c}12 \\
46.15 \%\end{array}$ & $\begin{array}{c}5 \\
19.23 \%\end{array}$ & $\begin{array}{c}6 \\
23.08 \%\end{array}$ & $\begin{array}{c}2 \\
7.69 \%\end{array}$ & $\begin{array}{c}1 \\
3.85 \%\end{array}$ & 26 \\
\hline Total \# & 34 & 32 & 38 & 13 & 3 & 120 \\
\hline \multicolumn{7}{|c|}{ Frequency Missing $=4$} \\
\hline
\end{tabular}

\begin{tabular}{|c|c|c|c|c|c|c|}
\hline \multicolumn{7}{|c|}{ Knowledge-Based Question } \\
\hline \multirow[t]{2}{*}{ Survey Period } & \multicolumn{6}{|c|}{ Some STDs Cannot Be Cured With Antibiotics. } \\
\hline & Strongly Agree & $\begin{array}{c}\text { Agree } \\
\text { Somewhat }\end{array}$ & Neutral & $\begin{array}{c}\text { Disagree } \\
\text { Somewhat }\end{array}$ & $\begin{array}{l}\text { Strongly } \\
\text { Disagree }\end{array}$ & Total \\
\hline Baseline & $\begin{array}{c}11 \\
11.58 \%\end{array}$ & $\begin{array}{c}17 \\
17.89 \%\end{array}$ & $\begin{array}{c}31 \\
32.63 \% \\
\end{array}$ & $\begin{array}{c}14 \\
14.74 \%\end{array}$ & $\begin{array}{c}22 \\
23.16 \%\end{array}$ & 95 \\
\hline 12-Month & $\begin{array}{c}6 \\
21.43 \% \\
\end{array}$ & $\begin{array}{c}3 \\
10.71 \%\end{array}$ & $\begin{array}{c}8 \\
28.57 \% \\
\end{array}$ & $\begin{array}{c}6 \\
21.43 \% \\
\end{array}$ & $\begin{array}{c}5 \\
17.86 \%\end{array}$ & 28 \\
\hline Total \# & 17 & 20 & 39 & 20 & 27 & 123 \\
\hline
\end{tabular}




\begin{tabular}{|c|c|c|c|c|c|c|}
\hline \multicolumn{7}{|c|}{ Knowledge-Based Question } \\
\hline \multirow[t]{2}{*}{ Survey Period } & \multicolumn{6}{|c|}{ Chronic/Recurrent Yeast Infections Are Signs Of HIV. } \\
\hline & Strongly Agree & $\begin{array}{c}\text { Agree } \\
\text { Somewhat }\end{array}$ & Neutral & $\begin{array}{c}\text { Disagree } \\
\text { Somewhat }\end{array}$ & $\begin{array}{l}\text { Strongly } \\
\text { Disagree }\end{array}$ & Total \\
\hline Baseline & $\begin{array}{c}8 \\
8.60 \% \\
\end{array}$ & $\begin{array}{c}20 \\
21.51 \%\end{array}$ & $\begin{array}{c}56 \\
60.22 \% \\
\end{array}$ & $\begin{array}{c}6 \\
6.45 \% \\
\end{array}$ & $\begin{array}{c}3 \\
3.23 \%\end{array}$ & 93 \\
\hline 12-Month & $\begin{array}{c}5 \\
17.86 \%\end{array}$ & $\begin{array}{c}6 \\
21.43 \%\end{array}$ & $\begin{array}{c}14 \\
50.00 \%\end{array}$ & $\begin{array}{c}1 \\
3.57 \%\end{array}$ & $\begin{array}{c}2 \\
7.14 \%\end{array}$ & 28 \\
\hline Total \# & 13 & 26 & 70 & 7 & 5 & 121 \\
\hline \multicolumn{7}{|c|}{ Frequency Missing = 3} \\
\hline
\end{tabular}

\begin{tabular}{|c|c|c|c|c|c|c|}
\hline \multicolumn{7}{|c|}{ Knowledge-Based Question } \\
\hline \multirow[t]{2}{*}{ Survey Period } & \multicolumn{6}{|c|}{ Sperm Can Live Up To 72 Hours Once Inside A Woman's Reproductive Tract. } \\
\hline & Strongly Agree & $\begin{array}{c}\text { Agree } \\
\text { Somewhat }\end{array}$ & Neutral & $\begin{array}{c}\text { Disagree } \\
\text { Somewhat }\end{array}$ & $\begin{array}{l}\text { Strongly } \\
\text { Disagree }\end{array}$ & Total \\
\hline Baseline & $\begin{array}{c}35 \\
36.84 \% \\
\end{array}$ & $\begin{array}{c}25 \\
26.32 \%\end{array}$ & $\begin{array}{c}29 \\
30.53 \% \\
\end{array}$ & $\begin{array}{c}3 \\
3.16 \%\end{array}$ & $\begin{array}{c}3 \\
3.16 \%\end{array}$ & 95 \\
\hline 12-Month & $\begin{array}{c}16 \\
57.14 \% \\
\end{array}$ & $\begin{array}{c}8 \\
28.57 \% \\
\end{array}$ & $\begin{array}{c}3 \\
10.71 \% \\
\end{array}$ & $\begin{array}{c}0 \\
0.00 \% \\
\end{array}$ & $\begin{array}{c}1 \\
3.57 \% \\
\end{array}$ & 28 \\
\hline Total \# & 51 & 33 & 32 & 3 & 4 & 123 \\
\hline \multicolumn{7}{|c|}{ Frequency Missing = 1} \\
\hline
\end{tabular}

\begin{tabular}{|c|c|c|c|c|c|c|}
\hline \multicolumn{7}{|c|}{ Knowledge-Based Question } \\
\hline \multirow[t]{2}{*}{ Survey Period } & \multicolumn{6}{|c|}{ Birth Control Pills Are 99\% Effective If Taken Correctly. } \\
\hline & Strongly Agree & $\begin{array}{c}\text { Agree } \\
\text { Somewhat }\end{array}$ & Neutral & $\begin{array}{c}\text { Disagree } \\
\text { Somewhat }\end{array}$ & $\begin{array}{l}\text { Strongly } \\
\text { Disagree }\end{array}$ & Total \\
\hline Baseline & $\begin{array}{c}49 \\
51.58 \%\end{array}$ & $\begin{array}{c}23 \\
24.21 \%\end{array}$ & $\begin{array}{c}14 \\
14.74 \%\end{array}$ & $\begin{array}{c}7 \\
7.37 \%\end{array}$ & $\begin{array}{c}2 \\
2.11 \%\end{array}$ & 95 \\
\hline 12-Month & $\begin{array}{c}22 \\
78.57 \%\end{array}$ & $\begin{array}{c}2 \\
7.14 \%\end{array}$ & $\begin{array}{c}1 \\
3.57 \%\end{array}$ & $\begin{array}{c}1 \\
3.57 \%\end{array}$ & $\begin{array}{c}2 \\
7.14 \%\end{array}$ & 28 \\
\hline Total \# & 71 & 25 & 15 & 8 & 4 & 123 \\
\hline
\end{tabular}

\begin{tabular}{|c|c|c|c|c|c|c|}
\hline \multicolumn{7}{|c|}{ Knowledge-Based Question } \\
\hline \multirow{2}{*}{ Survey Period } & \multicolumn{6}{|c|}{ Depo-Provera Is Birth Control That Consists Of A Hormone Injected Every 3 Months. } \\
\hline & Strongly Agree & $\begin{array}{c}\text { Agree } \\
\text { Somewhat }\end{array}$ & Neutral & $\begin{array}{c}\text { Disagree } \\
\text { Somewhat }\end{array}$ & $\begin{array}{l}\text { Strongly } \\
\text { Disagree }\end{array}$ & Total \\
\hline Baseline & $\begin{array}{c}39 \\
40.63 \%\end{array}$ & $\begin{array}{c}12 \\
12.50 \% \\
\end{array}$ & $\begin{array}{c}38 \\
39.58 \% \\
\end{array}$ & $\begin{array}{c}4 \\
4.17 \% \\
\end{array}$ & $\begin{array}{c}3 \\
3.13 \% \\
\end{array}$ & 96 \\
\hline 12-Month & $\begin{array}{c}21 \\
75.00 \% \\
\end{array}$ & $\begin{array}{c}5 \\
17.86 \% \\
\end{array}$ & $\begin{array}{c}1 \\
3.57 \% \\
\end{array}$ & $\begin{array}{c}0 \\
0.00 \% \\
\end{array}$ & $\begin{array}{c}1 \\
3.57 \% \\
\end{array}$ & 28 \\
\hline Total \# & 60 & 17 & 39 & 4 & 4 & 124 \\
\hline
\end{tabular}

\begin{tabular}{|l|c|c|c|c|c|c|}
\hline Knowledge-Based Question & \multicolumn{10}{|c|}{} \\
\hline Survey Period & The Implanon Method Of Birth Control Is Effective For Only A Year. \\
\hline & Strongly Agree & $\begin{array}{c}\text { Agree } \\
\text { Somewhat }\end{array}$ & Neutral & $\begin{array}{c}\text { Disagree } \\
\text { Somewhat }\end{array}$ & $\begin{array}{c}\text { Strongly } \\
\text { Disagree }\end{array}$ & Total \\
\hline Baseline & 6 & 6 & 73 & 4 & 7 & 96 \\
& $6.25 \%$ & $6.25 \%$ & $76.04 \%$ & $4.17 \%$ & $7.29 \%$ & 3 \\
\hline 12-Month & 5 & 4 & 15 & 1 & $3.71 \%$ & 28 \\
\hline Total \# & $17.86 \%$ & $14.29 \%$ & $53.57 \%$ & $3.57 \%$ & $10.71 \%$ & 124 \\
\hline
\end{tabular}




\begin{tabular}{|c|c|c|c|c|c|c|}
\hline \multicolumn{7}{|c|}{ Knowledge-Based Question } \\
\hline \multirow[t]{2}{*}{ Survey Period } & \multicolumn{6}{|c|}{ Withdrawal Of The Penis Before Ejaculation Is Effective To Prevent Pregnancy. } \\
\hline & Strongly Agree & $\begin{array}{c}\text { Agree } \\
\text { Somewhat }\end{array}$ & Neutral & $\begin{array}{c}\text { Disagree } \\
\text { Somewhat }\end{array}$ & $\begin{array}{l}\text { Strongly } \\
\text { Disagree }\end{array}$ & Total \\
\hline Baseline & $\begin{array}{c}8 \\
8.42 \% \\
\end{array}$ & $\begin{array}{c}13 \\
13.68 \% \\
\end{array}$ & $\begin{array}{c}9 \\
9.47 \% \\
\end{array}$ & $\begin{array}{c}25 \\
26.32 \% \\
\end{array}$ & $\begin{array}{c}40 \\
42.11 \%\end{array}$ & 95 \\
\hline 12-Month & $\begin{array}{c}3 \\
10.71 \% \\
\end{array}$ & $\begin{array}{c}4 \\
14.29 \%\end{array}$ & $\begin{array}{c}1 \\
3.57 \%\end{array}$ & $\begin{array}{c}6 \\
21.43 \% \\
\end{array}$ & $\begin{array}{c}14 \\
50.00 \%\end{array}$ & 28 \\
\hline Total \# & 11 & 17 & 10 & 31 & 54 & 123 \\
\hline \multicolumn{7}{|c|}{ Frequency Missing = 1} \\
\hline
\end{tabular}

\begin{tabular}{|c|c|c|c|c|c|c|}
\hline \multicolumn{7}{|c|}{ Knowledge-Based Question } \\
\hline \multirow[t]{2}{*}{ Survey Period } & \multicolumn{6}{|c|}{ A 12-Ounce Beer/4-5 Ounce Wine/1-2ounce Shot All Have The Same Amount Of Alcohol. } \\
\hline & Strongly Agree & $\begin{array}{c}\text { Agree } \\
\text { Somewhat }\end{array}$ & Neutral & $\begin{array}{c}\text { Disagree } \\
\text { Somewhat }\end{array}$ & $\begin{array}{l}\text { Strongly } \\
\text { Disagree }\end{array}$ & Total \\
\hline Baseline & $\begin{array}{c}34 \\
35.79 \% \\
\end{array}$ & $\begin{array}{c}17 \\
17.89 \% \\
\end{array}$ & $\begin{array}{c}35 \\
36.84 \% \\
\end{array}$ & $\begin{array}{c}1 \\
1.05 \%\end{array}$ & $\begin{array}{c}8 \\
8.42 \%\end{array}$ & 95 \\
\hline 12-Month & $\begin{array}{c}12 \\
42.86 \% \\
\end{array}$ & $\begin{array}{c}5 \\
17.86 \% \\
\end{array}$ & $\begin{array}{c}9 \\
32.14 \% \\
\end{array}$ & $\begin{array}{c}1 \\
3.57 \% \\
\end{array}$ & $\begin{array}{c}1 \\
3.57 \% \\
\end{array}$ & 28 \\
\hline Total \# & 46 & 22 & 44 & 2 & 9 & 123 \\
\hline
\end{tabular}

\begin{tabular}{|c|c|c|c|c|c|c|}
\hline \multicolumn{7}{|c|}{ Knowledge-Based Question } \\
\hline \multirow[t]{2}{*}{ Survey Period } & \multicolumn{6}{|c|}{$\begin{array}{l}\text { A Person Who Is Dehydrated Can Become Drunk Easier Than A Person Who Has Had Lots } \\
\text { Of Fluids. }\end{array}$} \\
\hline & Strongly Agree & $\begin{array}{c}\text { Agree } \\
\text { Somewhat }\end{array}$ & Neutral & $\begin{array}{l}\text { Disagree } \\
\text { Somewhat }\end{array}$ & $\begin{array}{l}\text { Strongly } \\
\text { Disagree }\end{array}$ & Total \\
\hline Baseline & $\begin{array}{c}46 \\
48.42 \%\end{array}$ & $\begin{array}{c}21 \\
22.11 \%\end{array}$ & $\begin{array}{c}18 \\
18.95 \%\end{array}$ & $\begin{array}{c}7 \\
7.37 \%\end{array}$ & $\begin{array}{c}3 \\
3.16 \%\end{array}$ & 95 \\
\hline 12-Month & $\begin{array}{c}16 \\
57.14 \%\end{array}$ & $\begin{array}{c}8 \\
28.57 \%\end{array}$ & $\begin{array}{c}3 \\
10.71 \%\end{array}$ & $\begin{array}{c}0 \\
0.00 \%\end{array}$ & $\begin{array}{c}1 \\
3.57 \%\end{array}$ & 28 \\
\hline Total \# & 62 & 29 & 21 & 7 & 4 & 123 \\
\hline
\end{tabular}

\begin{tabular}{|c|c|c|c|c|c|c|}
\hline \multicolumn{7}{|c|}{ Knowledge-Based Question } \\
\hline \multirow[t]{2}{*}{ Survey Period } & \multicolumn{6}{|c|}{$\begin{array}{l}\text { Alcohol Has The Same Effects On A Woman And A Man Who Weigh The Same And Drink } \\
\text { Same Amount. }\end{array}$} \\
\hline & Strongly Agree & $\begin{array}{c}\text { Agree } \\
\text { Somewhat }\end{array}$ & Neutral & $\begin{array}{l}\text { Disagree } \\
\text { Somewhat }\end{array}$ & $\begin{array}{l}\text { Strongly } \\
\text { Disagree }\end{array}$ & Total \\
\hline Baseline & $\begin{array}{c}6 \\
6.32 \%\end{array}$ & $\begin{array}{c}10 \\
10.53 \%\end{array}$ & $\begin{array}{c}23 \\
24.21 \%\end{array}$ & $\begin{array}{c}23 \\
24.21 \%\end{array}$ & 33 & 95 \\
\hline 12-Month & $\begin{array}{c}2 \\
7.14 \%\end{array}$ & $\begin{array}{c}0 \\
0.00 \%\end{array}$ & $\begin{array}{c}5 \\
17.86 \%\end{array}$ & $\begin{array}{c}5 \\
17.86 \%\end{array}$ & $\begin{array}{c}16 \\
57.14 \%\end{array}$ & 28 \\
\hline Total \# & 8 & 10 & 28 & 28 & 49 & 123 \\
\hline \multicolumn{7}{|c|}{ Frequency Missing = 1} \\
\hline
\end{tabular}

\begin{tabular}{|c|c|c|c|c|c|c|}
\hline \multicolumn{7}{|c|}{ Knowledge-Based Question } \\
\hline \multirow[t]{2}{*}{ Survey Period } & \multicolumn{6}{|c|}{ A Hungry Person Will Become Drunk Easier Than A Person Who Just Had A Full Meal. } \\
\hline & Strongly Agree & $\begin{array}{c}\text { Agree } \\
\text { Somewhat }\end{array}$ & Neutral & $\begin{array}{l}\text { Disagree } \\
\text { Somewhat }\end{array}$ & $\begin{array}{l}\text { Strongly } \\
\text { Disagree }\end{array}$ & Total \\
\hline Baseline & $\begin{array}{c}48 \\
50.53 \% \\
\end{array}$ & $\begin{array}{c}28 \\
29.47 \%\end{array}$ & $\begin{array}{c}12 \\
12.63 \%\end{array}$ & $\begin{array}{c}5 \\
5.26 \%\end{array}$ & $\begin{array}{c}2 \\
2.11 \%\end{array}$ & 95 \\
\hline 12-Month & $\begin{array}{c}19 \\
67.86 \% \\
\end{array}$ & $\begin{array}{c}5 \\
17.86 \%\end{array}$ & $\begin{array}{c}3 \\
10.71 \%\end{array}$ & $\begin{array}{c}0 \\
0.00 \%\end{array}$ & $\begin{array}{c}1 \\
3.57 \%\end{array}$ & 28 \\
\hline Total \# & 67 & 33 & 15 & 5 & 3 & 123 \\
\hline
\end{tabular}




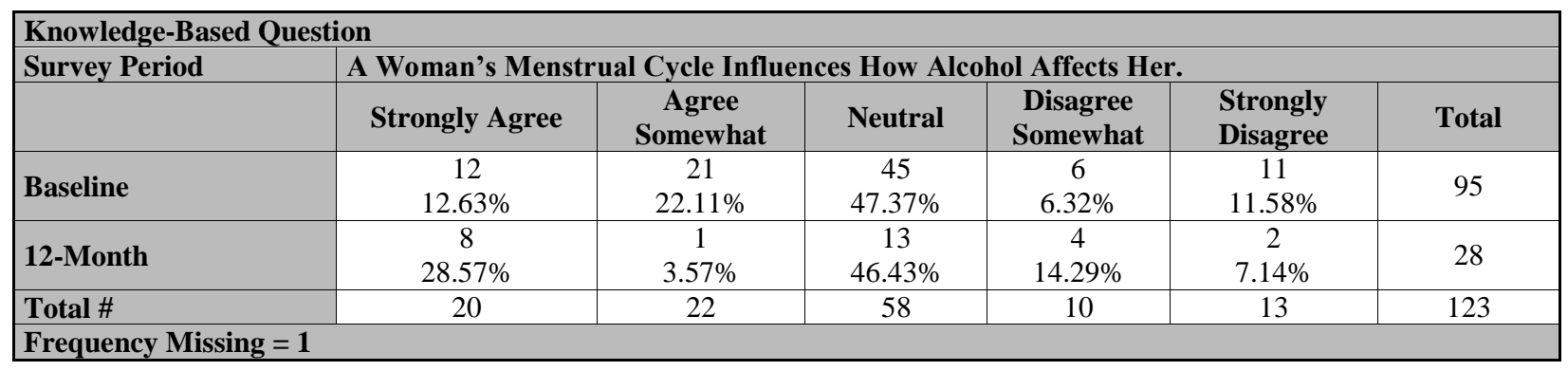

\begin{tabular}{|c|c|c|c|c|c|c|}
\hline \multicolumn{7}{|c|}{ Knowledge-Based Question } \\
\hline \multirow[t]{2}{*}{ Survey Period } & \multicolumn{6}{|c|}{ How Effective Are Birth-Control Pills In Preventing Pregnancy? } \\
\hline & Very Effective & $\begin{array}{l}\text { Somewhat } \\
\text { Effective }\end{array}$ & Neutral & $\begin{array}{l}\text { Somewhat } \\
\text { Ineffective }\end{array}$ & $\begin{array}{c}\text { Totally } \\
\text { Ineffective }\end{array}$ & Total \\
\hline Baseline & $\begin{array}{c}28 \\
29.79 \%\end{array}$ & $\begin{array}{c}53 \\
56.38 \%\end{array}$ & $\begin{array}{c}5 \\
5.32 \%\end{array}$ & $\begin{array}{c}8 \\
8.51 \%\end{array}$ & $\begin{array}{c}0 \\
0.00 \%\end{array}$ & 94 \\
\hline 12-Month & $\begin{array}{c}18 \\
64.29 \%\end{array}$ & $\begin{array}{c}8 \\
28.57 \%\end{array}$ & $\begin{array}{c}2 \\
7.14 \%\end{array}$ & $\begin{array}{c}0 \\
0.00 \%\end{array}$ & $\begin{array}{c}0 \\
0.00 \%\end{array}$ & 28 \\
\hline Total \# & 46 & 61 & 7 & 8 & 0 & 122 \\
\hline \multicolumn{7}{|c|}{ Frequency Missing = 2} \\
\hline
\end{tabular}

\begin{tabular}{|c|c|c|c|c|c|c|}
\hline \multicolumn{7}{|c|}{ Knowledge-Based Ouestion } \\
\hline \multirow[t]{2}{*}{ Survey Period } & \multicolumn{6}{|c|}{ How Effective Are Birth-Control Pills In Preventing STDs? } \\
\hline & Very Effective & $\begin{array}{c}\text { Somewhat } \\
\text { Effective }\end{array}$ & Neutral & $\begin{array}{l}\text { Somewhat } \\
\text { Ineffective }\end{array}$ & $\begin{array}{c}\text { Totally } \\
\text { Ineffective }\end{array}$ & Total \\
\hline Baseline & $\begin{array}{c}1 \\
1.05 \%\end{array}$ & $\begin{array}{c}10 \\
10.53 \%\end{array}$ & $\begin{array}{c}12 \\
12.63 \%\end{array}$ & $\begin{array}{c}9 \\
9.47 \%\end{array}$ & $\begin{array}{c}63 \\
66.32 \%\end{array}$ & 95 \\
\hline 12-Month & $\begin{array}{c}0 \\
0.00 \%\end{array}$ & $\begin{array}{c}1 \\
3.57 \%\end{array}$ & $\begin{array}{c}4 \\
14.29 \%\end{array}$ & $\begin{array}{c}2 \\
7.14 \%\end{array}$ & $\begin{array}{c}21 \\
75.00 \%\end{array}$ & 28 \\
\hline Total \# & 1 & 11 & 16 & 11 & 84 & 123 \\
\hline
\end{tabular}

\begin{tabular}{|c|c|c|c|c|c|c|}
\hline \multicolumn{7}{|c|}{ Knowledge-Based Question } \\
\hline \multirow[t]{2}{*}{ Survey Period } & \multicolumn{6}{|c|}{ How Effective Is The Depo-Provera In Preventing Pregnancy? } \\
\hline & $\begin{array}{c}\text { Very } \\
\text { Effective }\end{array}$ & $\begin{array}{l}\text { Somewhat } \\
\text { Effective }\end{array}$ & Neutral & $\begin{array}{l}\text { Somewhat } \\
\text { Ineffective }\end{array}$ & $\begin{array}{c}\text { Totally } \\
\text { Ineffective }\end{array}$ & Total \\
\hline Baseline & $\begin{array}{c}21 \\
22.11 \% \\
\end{array}$ & $\begin{array}{c}34 \\
35.79 \% \\
\end{array}$ & $\begin{array}{c}32 \\
33.68 \% \\
\end{array}$ & $\begin{array}{c}5 \\
5.26 \% \\
\end{array}$ & $\begin{array}{c}2 \\
2.11 \% \\
\end{array}$ & 95 \\
\hline 12-Month & $\begin{array}{c}9 \\
32.14 \%\end{array}$ & $\begin{array}{c}14 \\
50.00 \% \\
\end{array}$ & $\begin{array}{c}5 \\
17.86 \% \\
\end{array}$ & $\begin{array}{c}0 \\
0.00 \%\end{array}$ & $\begin{array}{c}0 \\
0.00 \% \\
\end{array}$ & 28 \\
\hline Total \# & 30 & 48 & 37 & 5 & 2 & 123 \\
\hline
\end{tabular}

\begin{tabular}{|c|c|c|c|c|c|}
\hline \multicolumn{6}{|c|}{ Knowledge-Based Question } \\
\hline \multirow[t]{2}{*}{ Survey Period } & \multicolumn{5}{|c|}{ How Effective Is The Depo-Provera In Preventing STDs? } \\
\hline & $\begin{array}{c}\text { Somewhat } \\
\text { Effective }\end{array}$ & Neutral & Somewhat Ineffective & $\begin{array}{c}\text { Totally } \\
\text { Ineffective }\end{array}$ & Total \\
\hline Baseline & $\begin{array}{c}5 \\
5.26 \%\end{array}$ & $\begin{array}{c}38 \\
40.00 \% \\
\end{array}$ & $\begin{array}{c}5 \\
5.26 \% \\
\end{array}$ & $\begin{array}{c}47 \\
49.47 \%\end{array}$ & 95 \\
\hline 12-Month & $\begin{array}{c}2 \\
7.14 \% \\
\end{array}$ & $\begin{array}{c}5 \\
17.86 \% \\
\end{array}$ & $\begin{array}{c}2 \\
7.14 \% \\
\end{array}$ & $\begin{array}{c}19 \\
67.86 \% \\
\end{array}$ & 28 \\
\hline Total \# & 7 & 43 & 7 & 66 & 123 \\
\hline
\end{tabular}




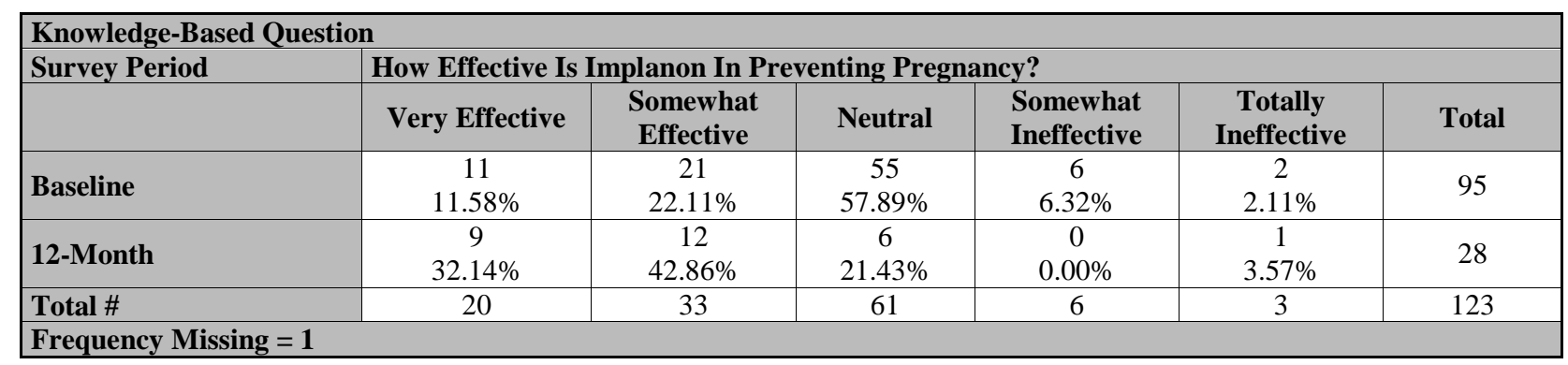

\begin{tabular}{|c|c|c|c|c|c|}
\hline \multicolumn{6}{|c|}{ Knowledge-Based Question } \\
\hline \multirow[t]{2}{*}{ Survey Period } & \multicolumn{5}{|c|}{ How Effective Is Implanon In Preventing STDs? } \\
\hline & $\begin{array}{c}\text { Somewhat } \\
\text { Effective }\end{array}$ & Neutral & $\begin{array}{l}\text { Somewhat } \\
\text { Ineffective }\end{array}$ & Totally Ineffective & Total \\
\hline Baseline & $\begin{array}{c}5 \\
5.26 \%\end{array}$ & $\begin{array}{c}50 \\
52.63 \%\end{array}$ & $\begin{array}{c}8 \\
8.42 \%\end{array}$ & $\begin{array}{c}32 \\
33.68 \%\end{array}$ & 95 \\
\hline 12-Month & $\begin{array}{c}2 \\
7.14 \%\end{array}$ & $\begin{array}{c}4 \\
14.29 \%\end{array}$ & $\begin{array}{c}2 \\
7.14 \%\end{array}$ & $\begin{array}{c}20 \\
71.43 \%\end{array}$ & 28 \\
\hline Total \# & 7 & 54 & 10 & 52 & 123 \\
\hline
\end{tabular}

\begin{tabular}{|c|c|c|c|c|c|c|}
\hline \multicolumn{7}{|c|}{ Knowledge-Based Question } \\
\hline \multirow[t]{2}{*}{ Survey Period } & \multicolumn{6}{|c|}{ How Effective Is Withdrawal Before Ejaculation In Preventing STDs? } \\
\hline & Very Effective & $\begin{array}{c}\text { Somewhat } \\
\text { Effective }\end{array}$ & Neutral & $\begin{array}{l}\text { Somewhat } \\
\text { Ineffective }\end{array}$ & Totally Ineffective & Total \\
\hline Baseline & $\begin{array}{c}3 \\
3.13 \% \\
\end{array}$ & $\begin{array}{c}10 \\
10.42 \% \\
\end{array}$ & $\begin{array}{c}12 \\
12.50 \% \\
\end{array}$ & $\begin{array}{c}21 \\
21.88 \% \\
\end{array}$ & $\begin{array}{c}50 \\
52.08 \% \\
\end{array}$ & 96 \\
\hline 12-Month & $\begin{array}{c}1 \\
3.70 \%\end{array}$ & $\begin{array}{c}4 \\
14.81 \%\end{array}$ & $\begin{array}{c}3 \\
11.11 \%\end{array}$ & $\begin{array}{c}5 \\
18.52 \%\end{array}$ & $\begin{array}{c}14 \\
51.85 \%\end{array}$ & 27 \\
\hline Total \# & 4 & 14 & 15 & 26 & 64 & 123 \\
\hline
\end{tabular}




\section{APPENDIX B: REPORTED BEHAVIORS}

\begin{tabular}{|c|c|c|c|c|c|c|c|}
\hline \multicolumn{8}{|c|}{ Reported Behaviors } \\
\hline \multirow[t]{2}{*}{ Survey Period } & \multicolumn{7}{|c|}{ In General, How Often Do You Use Birth Control To Prevent Pregnancy? } \\
\hline & Always & Usually & Sometimes & $\begin{array}{c}\text { Almost } \\
\text { Never }\end{array}$ & Never & $\begin{array}{c}\text { Does } \\
\text { not apply }\end{array}$ & Total \\
\hline Baseline & $\begin{array}{c}45 \\
49.45 \%\end{array}$ & $\begin{array}{c}8 \\
8.79 \%\end{array}$ & $\begin{array}{c}12 \\
13.19 \%\end{array}$ & $\begin{array}{c}3 \\
3.30 \% \\
\end{array}$ & $\begin{array}{c}10 \\
10.99 \% \\
\end{array}$ & $\begin{array}{c}12 \\
13.19 \% \\
\end{array}$ & 91 \\
\hline 4-Month & $\begin{array}{l}0 \\
\end{array}$ & 0 & 0 & $\begin{array}{l}0 \\
.\end{array}$ & $\begin{array}{l}0 \\
.\end{array}$ & 0 & 0 \\
\hline 12-Month & $\begin{array}{c}10 \\
38.46 \%\end{array}$ & $\begin{array}{c}5 \\
19.23 \%\end{array}$ & $\begin{array}{c}2 \\
7.69 \%\end{array}$ & $\begin{array}{c}1 \\
3.85 \% \\
\end{array}$ & $\begin{array}{c}3 \\
11.54 \%\end{array}$ & $\begin{array}{c}5 \\
19.23 \%\end{array}$ & 26 \\
\hline Total & 55 & 13 & 14 & 4 & 13 & 17 & 117 \\
\hline
\end{tabular}

\begin{tabular}{|c|c|c|c|c|c|c|c|}
\hline \multicolumn{8}{|c|}{ Reported Behaviors } \\
\hline \multirow[t]{2}{*}{ Survey Period } & \multicolumn{7}{|c|}{ In General, How Often Do You Use A Condom When You Have Sex? } \\
\hline & Always & $\begin{array}{c}\text { More than half } \\
\text { the time }\end{array}$ & $\begin{array}{c}\text { About half } \\
\text { the time }\end{array}$ & $\begin{array}{c}\text { Less than } \\
\text { half the time }\end{array}$ & Never & $\begin{array}{c}\text { Does } \\
\text { not apply }\end{array}$ & Total \\
\hline Baseline & 0 & $\begin{array}{l}0 \\
.\end{array}$ & 0 & 0 & 0 & 0 & 0 \\
\hline 4-Month & $\begin{array}{c}11 \\
28.95 \%\end{array}$ & $\begin{array}{c}7 \\
18.42 \%\end{array}$ & $\begin{array}{c}2 \\
5.26 \%\end{array}$ & $\begin{array}{c}6 \\
15.79 \%\end{array}$ & $\begin{array}{c}12 \\
31.58 \%\end{array}$ & $\begin{array}{c}0 \\
0.00 \%\end{array}$ & 38 \\
\hline 12-Month & $\begin{array}{c}4 \\
15.38 \%\end{array}$ & $\begin{array}{c}2 \\
7.69 \%\end{array}$ & $\begin{array}{c}3 \\
11.54 \%\end{array}$ & $\begin{array}{c}6 \\
23.08 \%\end{array}$ & $\begin{array}{c}6 \\
23.08 \%\end{array}$ & $\begin{array}{c}5 \\
19.23 \%\end{array}$ & 26 \\
\hline Total & 15 & 9 & 5 & 12 & 18 & 5 & 64 \\
\hline
\end{tabular}

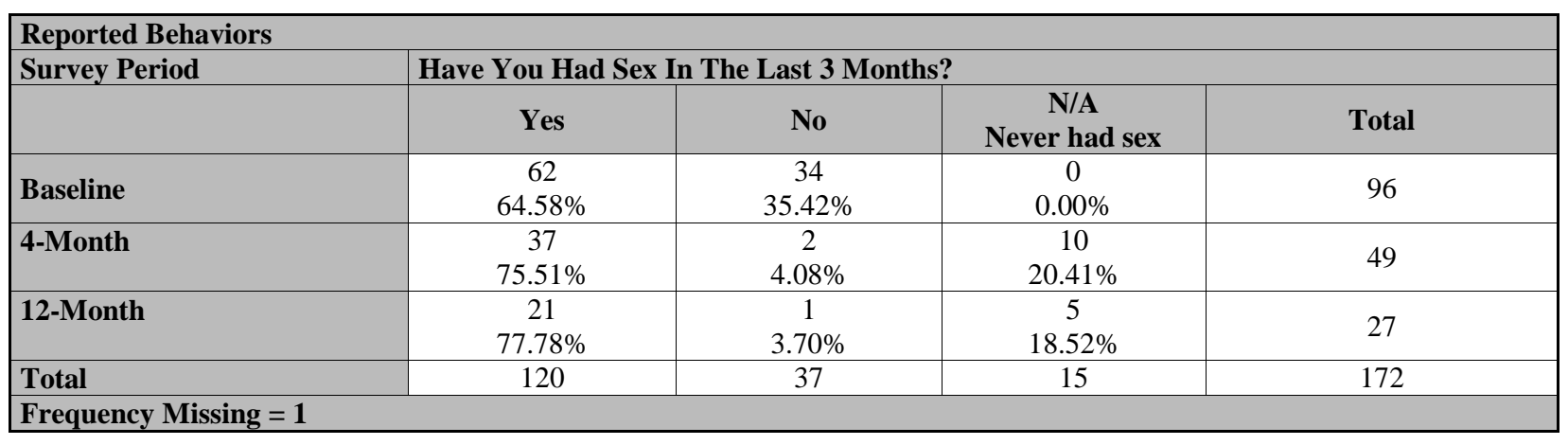

\begin{tabular}{|c|c|c|c|c|c|c|c|}
\hline \multicolumn{8}{|c|}{ Reported Behaviors } \\
\hline \multirow[t]{2}{*}{ Survey Period } & \multicolumn{7}{|c|}{ I Have Used Birth Control To Prevent Pregnancy In The Last 3 Months: } \\
\hline & Always & Usually & Sometimes & Almost Never & Never & Does not apply & Total \\
\hline Baseline & $\begin{array}{c}39 \\
45.35 \% \\
\end{array}$ & $\begin{array}{c}5 \\
5.81 \% \\
\end{array}$ & $\begin{array}{c}4 \\
4.65 \% \\
\end{array}$ & $\begin{array}{c}1 \\
1.16 \% \\
\end{array}$ & $\begin{array}{c}17 \\
19.77 \% \\
\end{array}$ & $\begin{array}{c}20 \\
23.26 \% \\
\end{array}$ & 86 \\
\hline 4-Month & $\begin{array}{c}25 \\
51.02 \%\end{array}$ & $\begin{array}{c}7 \\
14.29 \% \\
\end{array}$ & $\begin{array}{c}1 \\
2.04 \% \\
\end{array}$ & $\begin{array}{c}0 \\
0.00 \% \\
\end{array}$ & $\begin{array}{c}7 \\
14.29 \% \\
\end{array}$ & $\begin{array}{c}9 \\
18.37 \% \\
\end{array}$ & 49 \\
\hline 12-Month & 0 & $\begin{array}{l}0 \\
. \\
\end{array}$ & $\begin{array}{l}0 \\
.\end{array}$ & $\begin{array}{l}0 \\
.\end{array}$ & $\begin{array}{l}0 \\
\end{array}$ & $\begin{array}{l}0 \\
\end{array}$ & 0 \\
\hline Total & 64 & 12 & 5 & 1 & 24 & 29 & 135 \\
\hline
\end{tabular}




\begin{tabular}{|c|c|c|c|c|c|c|c|}
\hline \multicolumn{8}{|c|}{ Reported Behaviors } \\
\hline \multirow[t]{2}{*}{ Survey Period } & \multicolumn{7}{|c|}{ How Often Did You Use A Condom When Having Sex In The Past 3 Months? } \\
\hline & Always & Usually & Sometimes & $\begin{array}{l}\text { Almost } \\
\text { Never }\end{array}$ & Never & $\begin{array}{c}\text { Does not } \\
\text { apply }\end{array}$ & Total \\
\hline Baseline & $\begin{array}{c}14 \\
15.22 \% \\
\end{array}$ & $\begin{array}{c}8 \\
8.70 \%\end{array}$ & $\begin{array}{c}6 \\
6.52 \% \\
\end{array}$ & $\begin{array}{c}12 \\
13.04 \% \\
\end{array}$ & $\begin{array}{c}24 \\
26.09 \%\end{array}$ & $\begin{array}{c}28 \\
30.43 \% \\
\end{array}$ & 92 \\
\hline 4-Month & 0 & $\begin{array}{l}0 \\
.\end{array}$ & $\begin{array}{l}0 \\
.\end{array}$ & $\begin{array}{l}0 \\
.\end{array}$ & $\begin{array}{l}0 \\
.\end{array}$ & $\begin{array}{l}0 \\
.\end{array}$ & 0 \\
\hline 12-Month & 0 & 0 & 0 & 0 & 0 & 0 & 0 \\
\hline Total & 14 & 8 & 6 & 12 & 24 & 28 & 92 \\
\hline
\end{tabular}

\begin{tabular}{|c|c|c|c|c|c|}
\hline \multicolumn{6}{|c|}{ Reported Behaviors } \\
\hline \multirow[t]{2}{*}{ Survey Period } & \multicolumn{5}{|c|}{$\begin{array}{l}\text { In The Past } 3 \text { Months Or Since The Program, Have You Talked To Your Partner About Using } \\
\text { Birth Control To Prevent Pregnancy? }\end{array}$} \\
\hline & $\begin{array}{l}\text { Yes, } \\
\text { All }\end{array}$ & $\begin{array}{l}\text { Yes, } \\
\text { Some }\end{array}$ & $\begin{array}{l}\text { No, } \\
\text { None }\end{array}$ & $\begin{array}{c}\text { Does } \\
\text { not apply }\end{array}$ & Total \\
\hline Baseline & $\begin{array}{c}45 \\
51.72 \%\end{array}$ & $\begin{array}{c}14 \\
16.09 \%\end{array}$ & $\begin{array}{c}7 \\
8.05 \%\end{array}$ & $\begin{array}{c}21 \\
24.14 \%\end{array}$ & 87 \\
\hline 4-Month & $\begin{array}{c}26 \\
55.32 \%\end{array}$ & $\begin{array}{c}4 \\
8.51 \%\end{array}$ & $\begin{array}{c}5 \\
10.64 \%\end{array}$ & $\begin{array}{c}12 \\
25.53 \%\end{array}$ & 47 \\
\hline 12-Month & $\begin{array}{c}15 \\
55.56 \%\end{array}$ & $\begin{array}{c}2 \\
7.41 \%\end{array}$ & $\begin{array}{c}4 \\
14.81 \%\end{array}$ & $\begin{array}{c}6 \\
22.22 \%\end{array}$ & 27 \\
\hline Total & 86 & 20 & 16 & 39 & 161 \\
\hline
\end{tabular}

\begin{tabular}{|c|c|c|c|c|c|}
\hline \multicolumn{6}{|c|}{ Reported Behaviors } \\
\hline \multirow[t]{2}{*}{ Survey Period } & \multicolumn{5}{|c|}{$\begin{array}{l}\text { In The Past } 3 \text { Months Or Since The Program, Have You Talked To Your Partners About Using } \\
\text { A Condom During Sex? }\end{array}$} \\
\hline & $\begin{array}{l}\text { Yes, } \\
\text { All }\end{array}$ & $\begin{array}{c}\text { Yes, } \\
\text { Some }\end{array}$ & $\begin{array}{c}\text { No, } \\
\text { None }\end{array}$ & $\begin{array}{c}\text { Does } \\
\text { not apply }\end{array}$ & Total \\
\hline Baseline & $\begin{array}{c}38 \\
43.68 \%\end{array}$ & $\begin{array}{c}10 \\
11.49 \%\end{array}$ & $\begin{array}{c}17 \\
19.54 \%\end{array}$ & $\begin{array}{c}22 \\
25.29 \%\end{array}$ & 87 \\
\hline 4-Month & $\begin{array}{c}23 \\
48.94 \%\end{array}$ & $\begin{array}{c}1 \\
2.13 \% \\
\end{array}$ & $\begin{array}{c}12 \\
25.53 \%\end{array}$ & $\begin{array}{c}11 \\
23.40 \%\end{array}$ & 47 \\
\hline 12-Month & $\begin{array}{c}12 \\
44.44 \%\end{array}$ & $\begin{array}{c}3 \\
11.11 \%\end{array}$ & $\begin{array}{c}6 \\
22.22 \%\end{array}$ & $\begin{array}{c}6 \\
22.22 \%\end{array}$ & 27 \\
\hline Total & 73 & 14 & 35 & 39 & 161 \\
\hline \multicolumn{6}{|c|}{ Frequency Missing = 12} \\
\hline
\end{tabular}

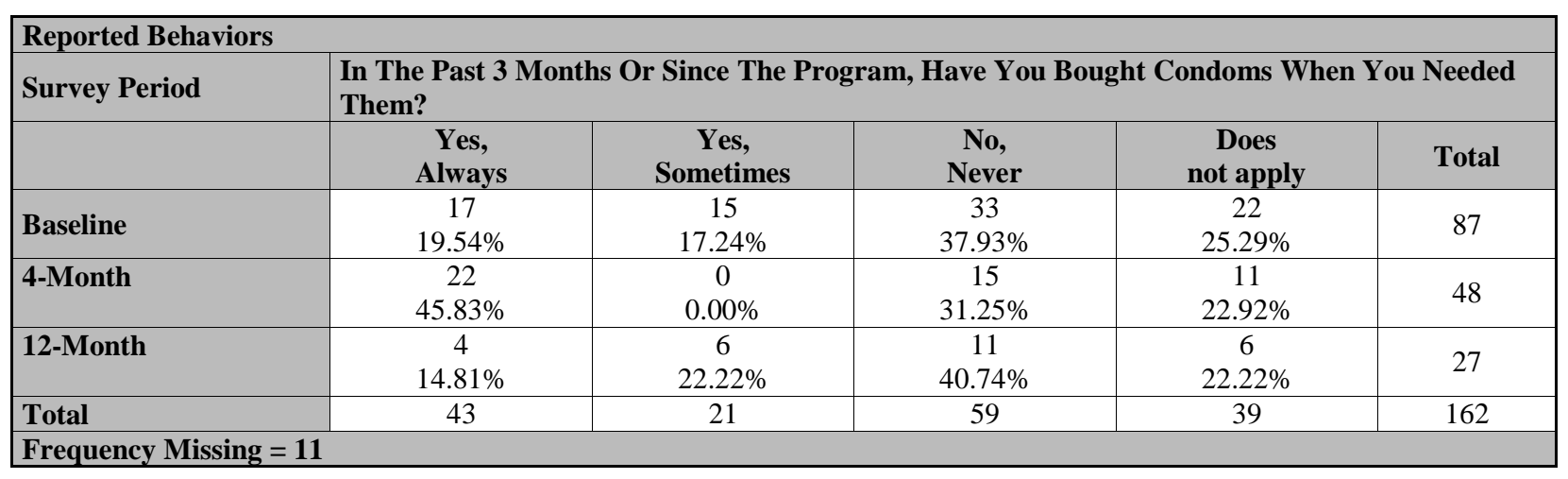




\begin{tabular}{|l|c|c|c|c|}
\hline Reported Behaviors & \multicolumn{5}{|c|}{$\begin{array}{l}\text { Have You Visited A Clinic/Healthcare Provider To Help You Choose The Right } \\
\text { Type Of Birth Control? }\end{array}$} \\
\hline Survey Period & Yes & No & $\begin{array}{c}\text { Does } \\
\text { not apply }\end{array}$ & Total \\
\hline Baseline & 22 & 44 & 20 & 86 \\
\hline 4-Month & $25.58 \%$ & $51.16 \%$ & $23.26 \%$ & 21 \\
\hline 12-Month & 8 & 9 & 4 & $2.05 \%$ \\
\hline Total & $38.10 \%$ & $42.86 \%$ & 6 & $22 \%$ \\
\hline Frequency Missing =39 & 9 & 12 & 30 & \\
\hline
\end{tabular}

\begin{tabular}{|c|c|c|c|c|}
\hline \multicolumn{5}{|c|}{ Reported Behaviors } \\
\hline \multirow[t]{2}{*}{ Survey Period } & \multicolumn{4}{|c|}{$\begin{array}{l}\text { Have You Gone To A Clinic/Healthcare Provider To Get Checked For An STD } \\
\text { Even If No Symptoms Were Present? }\end{array}$} \\
\hline & Yes & No & $\begin{array}{c}\text { Does } \\
\text { not apply }\end{array}$ & Total \\
\hline Baseline & $\begin{array}{c}19 \\
22.09 \%\end{array}$ & $\begin{array}{c}45 \\
52.33 \%\end{array}$ & $\begin{array}{c}22 \\
25.58 \%\end{array}$ & 86 \\
\hline 4-Month & 0 & 0 & 0 & 0 \\
\hline 12-Month & $\begin{array}{c}15 \\
55.56 \%\end{array}$ & $\begin{array}{c}6 \\
22.22 \% \\
\end{array}$ & $\begin{array}{c}6 \\
22.22 \% \\
\end{array}$ & 27 \\
\hline Total & 34 & 51 & 28 & 113 \\
\hline
\end{tabular}

\begin{tabular}{|c|c|c|c|c|c|c|c|}
\hline \multicolumn{8}{|c|}{ Reported Behaviors } \\
\hline \multirow{2}{*}{ Survey Period } & \multicolumn{7}{|c|}{ How Many Times Have You Had Sex After Drinking Alcohol? } \\
\hline & None & Once & 2-3 times & 4 or more & Always & $\begin{array}{c}\text { Does } \\
\text { not apply }\end{array}$ & Total \\
\hline Baseline & $\begin{array}{c}35 \\
38.04 \%\end{array}$ & $\begin{array}{c}8 \\
8.70 \%\end{array}$ & $\begin{array}{c}12 \\
13.04 \%\end{array}$ & $\begin{array}{c}8 \\
8.70 \%\end{array}$ & $\begin{array}{c}2 \\
2.17 \%\end{array}$ & $\begin{array}{c}27 \\
29.35 \%\end{array}$ & 92 \\
\hline 4-Month & $\begin{array}{c}27 \\
55.10 \%\end{array}$ & $\begin{array}{c}7 \\
14.29 \%\end{array}$ & $\begin{array}{c}9 \\
18.37 \%\end{array}$ & $\begin{array}{c}6 \\
12.24 \%\end{array}$ & $\begin{array}{c}0 \\
0.00 \%\end{array}$ & $\begin{array}{c}0 \\
0.00 \%\end{array}$ & 49 \\
\hline 12-Month & $\begin{array}{c}16 \\
59.26 \%\end{array}$ & $\begin{array}{c}2 \\
7.41 \%\end{array}$ & $\begin{array}{c}6 \\
22.22 \%\end{array}$ & $\begin{array}{c}3 \\
11.11 \%\end{array}$ & $\begin{array}{c}0 \\
0.00 \%\end{array}$ & $\begin{array}{c}0 \\
0.00 \%\end{array}$ & 27 \\
\hline Total & 78 & 17 & 27 & 17 & 2 & 27 & 168 \\
\hline
\end{tabular}

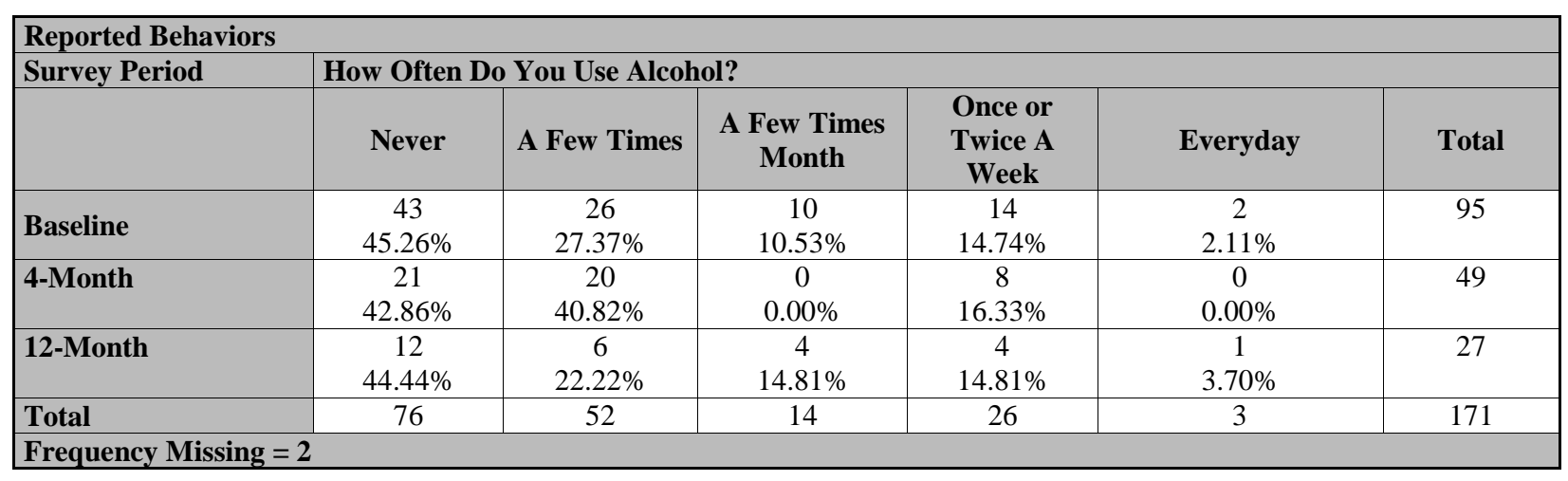




\begin{tabular}{|c|c|c|c|c|c|c|}
\hline \multicolumn{7}{|c|}{ Reported Behaviors } \\
\hline \multirow{2}{*}{ Survey Period } & \multicolumn{6}{|c|}{ How Often Do You Use Drugs? } \\
\hline & Never & A Few Times & $\begin{array}{c}\text { A Few Times } \\
\text { Month }\end{array}$ & $\begin{array}{c}\text { Once or } \\
\text { Twice A } \\
\text { Week }\end{array}$ & Everyday & Total \\
\hline Baseline & $\begin{array}{c}76 \\
80.00 \%\end{array}$ & $\begin{array}{c}9 \\
9.47 \%\end{array}$ & $\begin{array}{c}2 \\
2.11 \%\end{array}$ & $\begin{array}{c}2 \\
2.11 \%\end{array}$ & $\begin{array}{c}6 \\
6.32 \%\end{array}$ & 95 \\
\hline 4-Month & 0 & 0 & 0 & 0 & 0 & 0 \\
\hline 12-Month & $\begin{array}{l}0 \\
\text {. }\end{array}$ & $\begin{array}{l}0 \\
\text {. }\end{array}$ & $\begin{array}{l}0 \\
\text {. }\end{array}$ & $\begin{array}{l}0 \\
\text {. }\end{array}$ & $\begin{array}{l}0 \\
\text {. }\end{array}$ & 0 \\
\hline Total & 76 & 9 & 2 & 2 & 6 & 95 \\
\hline \multicolumn{7}{|c|}{ Frequency Missing $=78$} \\
\hline
\end{tabular}

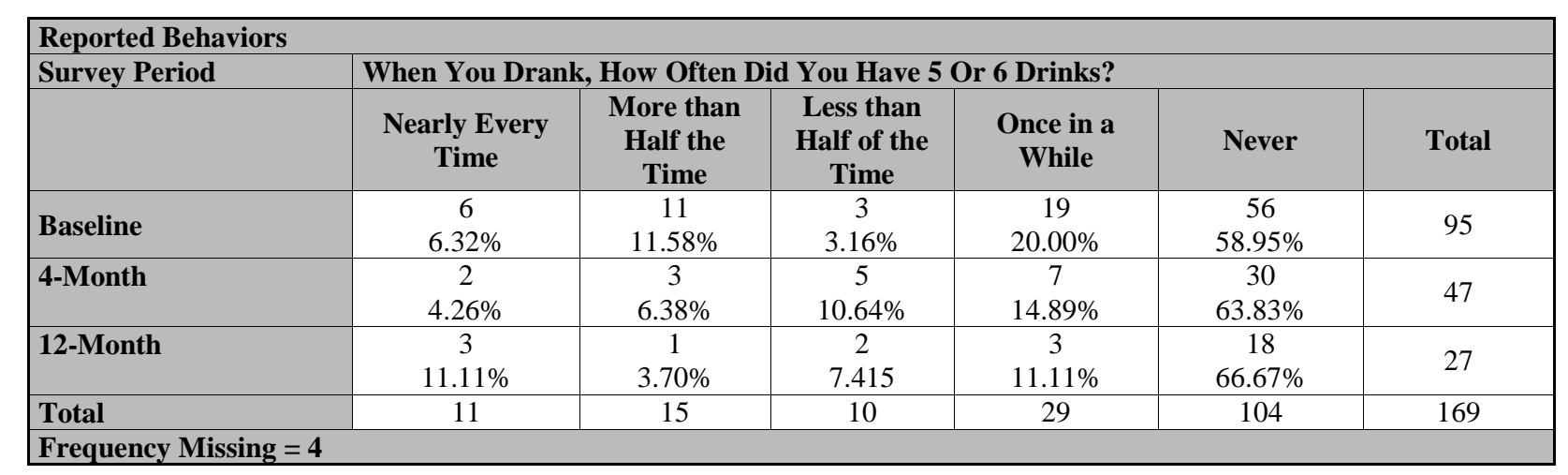

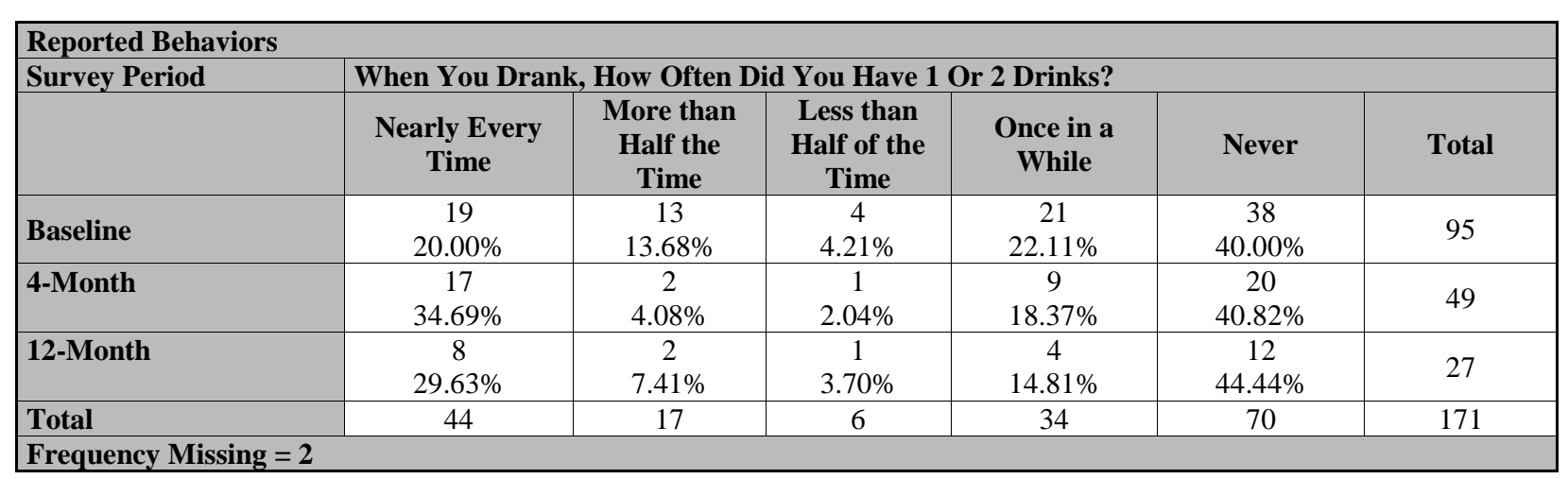

\begin{tabular}{|c|c|c|c|c|c|c|}
\hline \multicolumn{7}{|c|}{ Reported Behaviors } \\
\hline \multirow[t]{2}{*}{ Survey Period } & \multicolumn{6}{|c|}{ When You Drank, How Often Did You Have 3 Or 4 Drinks? } \\
\hline & $\begin{array}{c}\text { Nearly } \\
\text { Every Time }\end{array}$ & $\begin{array}{c}\text { More than } \\
\text { Half the } \\
\text { Time }\end{array}$ & $\begin{array}{l}\text { Less than } \\
\text { Half of the } \\
\text { Time }\end{array}$ & $\begin{array}{l}\text { Once in a } \\
\text { While }\end{array}$ & Never & Total \\
\hline Baseline & $\begin{array}{l}0 \\
.\end{array}$ & 0 & 0 & 0 & 0 & 0 \\
\hline 4-Month & $\begin{array}{c}3 \\
6.12 \%\end{array}$ & $\begin{array}{c}4 \\
8.16 \%\end{array}$ & $\begin{array}{c}8 \\
16.33 \%\end{array}$ & $\begin{array}{c}8 \\
16.33 \%\end{array}$ & $\begin{array}{c}26 \\
53.06 \%\end{array}$ & 49 \\
\hline 12-Month & $\begin{array}{c}3 \\
11.54 \%\end{array}$ & $\begin{array}{c}1 \\
3.85 \%\end{array}$ & $\begin{array}{c}4 \\
15.38 \%\end{array}$ & $\begin{array}{c}4 \\
15.38 \%\end{array}$ & $\begin{array}{c}14 \\
53.85 \%\end{array}$ & 26 \\
\hline Total & 6 & 5 & 12 & 12 & 40 & 75 \\
\hline
\end{tabular}




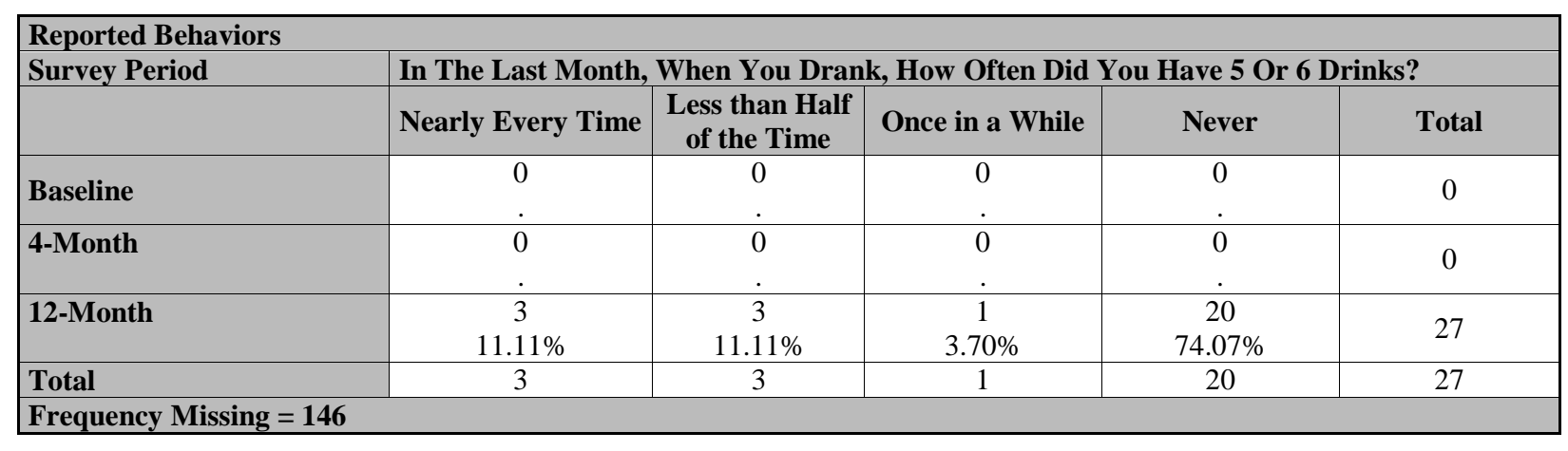

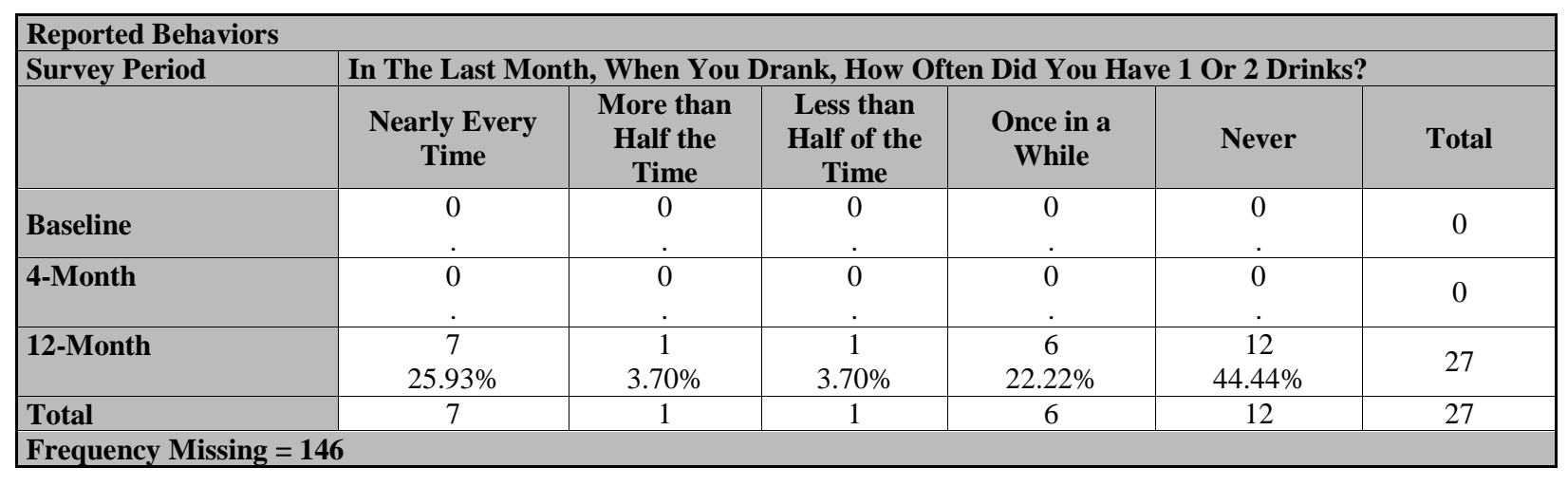

\begin{tabular}{|c|c|c|c|c|c|c|}
\hline \multicolumn{7}{|c|}{ Reported Behaviors } \\
\hline \multirow[t]{2}{*}{ Survey Period } & \multicolumn{6}{|c|}{ In The Last Month, When You Drank, How Often Did You Have 3 Or 4 Drinks? } \\
\hline & $\begin{array}{c}\text { Nearly Every } \\
\text { Time }\end{array}$ & $\begin{array}{l}\text { More than } \\
\text { Half the } \\
\text { Time }\end{array}$ & $\begin{array}{c}\text { Less than } \\
\text { Half of the } \\
\text { Time }\end{array}$ & $\begin{array}{c}\text { Once in a } \\
\text { While }\end{array}$ & Never & Total \\
\hline Baseline & 0 & 0 & 0 & 0 & 0 & 0 \\
\hline 4-Month & 0 & 0 & 0 & 0 & 0 & 0 \\
\hline 12-Month & $\begin{array}{c}2 \\
7.41 \% \\
\end{array}$ & $\begin{array}{c}4 \\
14.81 \%\end{array}$ & $\begin{array}{c}1 \\
3.70 \%\end{array}$ & $\begin{array}{c}4 \\
14.81 \%\end{array}$ & $\begin{array}{c}16 \\
59.26 \%\end{array}$ & 27 \\
\hline Total & 2 & 4 & 1 & 4 & 16 & 27 \\
\hline
\end{tabular}

\begin{tabular}{|c|c|c|c|c|c|}
\hline \multicolumn{6}{|c|}{ Reported Behaviors } \\
\hline \multirow[t]{2}{*}{ Survey Period } & \multicolumn{5}{|c|}{ Have You Had Oral Sex? } \\
\hline & $\begin{array}{l}\text { Yes, Used no } \\
\text { barrier }\end{array}$ & Yes, Used barrier & $\begin{array}{c}\text { No, No oral sex } \\
\text { during this } \\
\text { time }\end{array}$ & No, Never & Total \\
\hline Baseline & 0 & 0 & 0 & 0 & 0 \\
\hline 4-Month & $\begin{array}{c}27 \\
55.10 \%\end{array}$ & $\begin{array}{c}1 \\
2.04 \%\end{array}$ & $\begin{array}{c}9 \\
18.37 \%\end{array}$ & $\begin{array}{c}12 \\
24.49 \%\end{array}$ & 49 \\
\hline 12-Month & 0 & 0 & 0 & 0 & 0 \\
\hline Total & 27 & 1 & 9 & 12 & 49 \\
\hline
\end{tabular}




\begin{tabular}{|l|c|c|c|}
\hline Reported Behaviors & \multicolumn{3}{|c|}{ Did You Use Birth Control When You Last Had Sex? } \\
\hline Survey Period & Method Specified & Does Not Apply & Total \\
\hline Baseline & 78 & 18 & 96 \\
\hline 4-Month & $81.25 \%$ & $18.75 \%$ & 49 \\
& 37 & 12 & 28 \\
\hline 12-Month & $75.51 \%$ & $24.49 \%$ & $21.43 \%$ \\
\hline Total & 22 & 36 & 2 \\
\hline
\end{tabular}

\begin{tabular}{|l|c|c|c|}
\hline Reported Behaviors & \multicolumn{3}{|c|}{ I Use No Method Of Protection When I Have Sex. } \\
\hline Survey Period & Not Selected & No Method Used & Total \\
\hline \multirow{2}{*}{ Baseline } & 86 & 10 & 96 \\
\hline 4-Month & $89.58 \%$ & $10.42 \%$ & 49 \\
& 45 & 4 & \multirow{2}{*}{2} \\
\hline 12-Month & $91.84 \%$ & $3.16 \%$ & 28 \\
\hline Total & 25 & $10.71 \%$ & 173 \\
\hline
\end{tabular}

\begin{tabular}{|c|c|c|c|}
\hline \multicolumn{4}{|c|}{ Reported Behaviors } \\
\hline \multirow[t]{2}{*}{ Survey Period } & \multicolumn{3}{|l|}{ I Use The Pill. } \\
\hline & Not Selected & Yes & Total \\
\hline Baseline & $\begin{array}{c}65 \\
67.71 \% \\
\end{array}$ & $\begin{array}{c}31 \\
32.29 \% \\
\end{array}$ & 96 \\
\hline 4-Month & $\begin{array}{c}33 \\
67.35 \% \\
\end{array}$ & $\begin{array}{c}16 \\
32.65 \% \\
\end{array}$ & 49 \\
\hline 12-Month & $\begin{array}{c}19 \\
67.86 \% \\
\end{array}$ & $\begin{array}{c}9 \\
32.14 \% \\
\end{array}$ & 28 \\
\hline Total & 117 & 56 & 173 \\
\hline
\end{tabular}

\begin{tabular}{|c|c|c|c|}
\hline \multicolumn{4}{|c|}{ Reported Behaviors } \\
\hline \multirow[t]{2}{*}{ Survey Period } & \multicolumn{3}{|c|}{ I Use Depo-Provera Or Implanon. } \\
\hline & Not Selected & Yes & Total \\
\hline Baseline & $\begin{array}{c}83 \\
86.46 \% \\
\end{array}$ & $\begin{array}{c}13 \\
13.54 \% \\
\end{array}$ & 96 \\
\hline 4-Month & $\begin{array}{c}44 \\
89.80 \%\end{array}$ & $\begin{array}{c}5 \\
10.20 \%\end{array}$ & 49 \\
\hline 12-Month & $\begin{array}{c}27 \\
96.43 \%\end{array}$ & $\begin{array}{c}1 \\
3.57 \%\end{array}$ & 28 \\
\hline Total & 154 & 19 & 173 \\
\hline
\end{tabular}

\begin{tabular}{|l|c|c|c|}
\hline Reported Behaviors & \multicolumn{3}{|c|}{ I Use Condoms With Spermicide When I Have Sex. } \\
\hline Survey Period & Not Selected & Yes & Total \\
\hline Baseline & 84 & 12 & 96 \\
\hline 4-Month & $87.50 \%$ & 0 & 0 \\
& 0 & 4 & $28 \%$ \\
\hline 12-Month &. & $14.29 \%$ & 28 \\
\hline Total & $85.71 \%$ & 16 & 124 \\
\hline Frequency Missing $=\mathbf{4 9}$ & 108 & & \multirow{2}{*}{} \\
\hline
\end{tabular}




\begin{tabular}{|l|c|c|c|}
\hline Reported Behaviors & \multicolumn{3}{|c|}{ I Use Only Condoms When I Have Sex. } \\
\hline Survey Period & Not Selected & 36 & Yes \\
\hline \multirow{2}{*}{ Baseline } & 60 & $37.50 \%$ & 96 \\
\hline 4-Month & $62.50 \%$ & 18 & 49 \\
& 31 & $36.73 \%$ & 28 \\
\hline 12-Month & $63.27 \%$ & 7 & $25.00 \%$ \\
\hline Total & 21 & 61 & 28 \\
\hline
\end{tabular}

\begin{tabular}{|l|c|c|c|}
\hline Reported Behaviors & \multicolumn{3}{|c|}{ I Use Only Spermicide When I Have Sex. } \\
\hline Survey Period & Not Selected & Yes & Total \\
\hline \multirow{2}{*}{ Baseline } & 96 & 0 & 96 \\
\hline 4-Month & $100.00 \%$ & $0.00 \%$ & 49 \\
& 49 & 0 & 28 \\
\hline 12-Month & $100.00 \%$ & 1 & $2.00 \%$ \\
\hline Total & 27 & $3.57 \%$ & 28 \\
\hline
\end{tabular}

\begin{tabular}{|l|c|c|c|}
\hline Reported Behaviors & \multicolumn{3}{|c|}{ I Use Either The Withdrawal Or The Rhythm Method When I Have Sex. } \\
\hline Survey Period & Not Selected & Yes & Total \\
\hline \multirow{2}{*}{ Baseline } & 73 & 23 & 96 \\
\hline 4-Month & $76.04 \%$ & $23.96 \%$ & 49 \\
& 39 & 10 & 28 \\
\hline 12-Month & $79.59 \%$ & 12 & $28 \%$ \\
\hline Total & 16 & $42.86 \%$ & 45 \\
\hline
\end{tabular}

\begin{tabular}{|l|c|c|c|}
\hline Reported Behaviors & \multicolumn{4}{|l|}{$\begin{array}{l}\text { In The Past 3 Months Or Since The Program, Did You Not Have Sex In Order To } \\
\text { Reduce The Risk Of Pregnancy And STDs? }\end{array}$} \\
\hline & Not Selected & Yes (Did Not Have Sex) & Total \\
\hline Baseline & 63 & 32 & 95 \\
\hline 4-Month & $66.32 \%$ & $33.68 \%$ & 49 \\
& 35 & 14 & $28.57 \%$ \\
\hline 12-Month & $71.43 \%$ & 6 & 172 \\
\hline Total & 22 & $21.43 \%$ & 28 \\
\hline Frequency Missing =1 & $78.57 \%$ & & \\
\hline
\end{tabular}

\begin{tabular}{|l|c|c|c|}
\hline Reported Behaviors & \multicolumn{3}{|c|}{$\begin{array}{l}\text { In The Past 3 Months Or Since The Program, Did You Reduce Your Number Of } \\
\text { Partners To Reduce Risk? }\end{array}$} \\
\hline Survey Period & Not Selected & Yes (Reduced Number) & Total \\
\hline Baseline & 80 & 15 & 95 \\
\hline 4-Month & $43.21 \%$ & $15.79 \%$ & 49 \\
& $87.76 \%$ & $12.24 \%$ & 28 \\
\hline 12-Month & 24 & 4 & $14.29 \%$ \\
\hline Total & $85.71 \%$ & 25 & 172 \\
\hline Frequency Missing =1 & 147 & & \\
\hline
\end{tabular}




\begin{tabular}{|l|c|c|c|}
\hline Reported Behaviors & \multicolumn{3}{|c|}{ In The Past 3 Months Or Since The Program, Have You Used Pills Or Hormonal } \\
Methods To Reduce Risk?
\end{tabular}

\begin{tabular}{|c|c|c|c|}
\hline \multicolumn{4}{|c|}{ Reported Behaviors } \\
\hline \multirow[t]{2}{*}{ Survey Period } & \multicolumn{3}{|c|}{$\begin{array}{l}\text { In The Past } 3 \text { Months Or Since The Program, Have You Used To Condoms To } \\
\text { Reduce Your Risk Of Pregnancy And STDs? }\end{array}$} \\
\hline & Not Selected & Yes & Total \\
\hline Baseline & $\begin{array}{c}64 \\
67.37 \%\end{array}$ & $\begin{array}{c}31 \\
32.63 \%\end{array}$ & 95 \\
\hline 4-Month & $\begin{array}{c}32 \\
65.31 \%\end{array}$ & $\begin{array}{c}17 \\
34.69 \%\end{array}$ & 49 \\
\hline 12-Month & $\begin{array}{c}14 \\
50.00 \%\end{array}$ & $\begin{array}{c}14 \\
50.00 \%\end{array}$ & 28 \\
\hline Total & 110 & 62 & 172 \\
\hline
\end{tabular}

\begin{tabular}{|l|c|c|c|}
\hline Reported Behaviors & \multicolumn{3}{|c|}{$\begin{array}{l}\text { In Order To Reduce Risk, In The Past 3 Months Or Since The Program, Have You } \\
\text { Refused To Have Sex If Your Partner Did Not Wear A Condom? }\end{array}$} \\
\hline \multirow{2}{*}{ Burvey Period } & Not Selected & Yes & Total \\
\hline 4-Month & 84 & 11 & 95 \\
& $88.42 \%$ & $11.58 \%$ & 49 \\
\hline 12-Month & 38 & 11 & 28 \\
\hline Total & $77.55 \%$ & 4 & $14.29 \%$ \\
\hline Frequency Missing = & 24 & 26 & 172 \\
\hline
\end{tabular}

\begin{tabular}{|l|c|c|c|}
\hline Reported Behaviors & \multicolumn{3}{|c|}{$\begin{array}{l}\text { In Order To Reduce Risk, In The Past 3 Months Or Since The Program, Have You } \\
\text { Only Had Sex With A Steady Partner? }\end{array}$} \\
\hline Survey Period & Not Selected & Yes & Total \\
\hline & 48 & 47 & 95 \\
\hline Baseline & $50.53 \%$ & $49.47 \%$ & 49 \\
\hline 4-Month & 25 & 24 & 28 \\
\hline 12-Month & $51.02 \%$ & 16 & 172 \\
\hline Total & 12 & $57.14 \%$ & \\
\hline Frequency Missing = & $42.86 \%$ & 87 & \\
\hline
\end{tabular}




\begin{tabular}{|l|c|c|c|}
\hline Reported Behaviors & \multicolumn{3}{|c|}{$\begin{array}{l}\text { To Reduce Risk, In The Past 3 Months Or Since The Program, Have You Used Only } \\
\text { Spermicide With No Other Form Of Protection? }\end{array}$} \\
\hline & Did Not Select & Yes & Total \\
\hline Baseline & 95 & 0 & 95 \\
\hline 4-Month & $100.00 \%$ & $0.00 \%$ & 49 \\
\hline 12-Month & 49 & 0 & 28 \\
\hline Total & $100.00 \%$ & 0 & 172 \\
\hline Frequency Missing =1 & 28 & $0.00 \%$ & 0 \\
\hline
\end{tabular}

\begin{tabular}{|l|c|c|c|}
\hline Reported Behaviors & \multicolumn{3}{|c|}{$\begin{array}{l}\text { To Reduce Risk, In The Past 3 Months Or Since The Program, Did You Use The } \\
\text { Withdrawal Or Rhythm Method? }\end{array}$} \\
\hline & Did Not Select & Yes & Total \\
\hline Baseline & 75 & 20 & 95 \\
\hline 4-Month & $78.95 \%$ & $21.05 \%$ & 49 \\
& 38 & 11 & $22.45 \%$ \\
\hline 12-Month & $77.55 \%$ & 12 & $28 \%$ \\
\hline Total & 16 & $42.86 \%$ & 172 \\
\hline Frequency Missing =1 & $57.14 \%$ & & \\
\hline
\end{tabular}

\begin{tabular}{|c|c|c|c|}
\hline \multicolumn{4}{|c|}{ Reported Behaviors } \\
\hline \multirow[t]{2}{*}{ Survey Period } & \multicolumn{3}{|c|}{$\begin{array}{l}\text { To Reduce Risk In The Past } 3 \text { Months Or Since The Program, Did You Ask Your } \\
\text { Partners About Their Sexual Histories? }\end{array}$} \\
\hline & \begin{tabular}{|l|} 
Did Not Select \\
\end{tabular} & Yes (I Asked) & Total \\
\hline Baseline & $\begin{array}{c}85 \\
89.47 \%\end{array}$ & $\begin{array}{c}10 \\
10.53 \%\end{array}$ & 95 \\
\hline 4-Month & $\begin{array}{c}42 \\
85.71 \% \\
\end{array}$ & $\begin{array}{c}7 \\
14.29 \% \\
\end{array}$ & 49 \\
\hline 12-Month & $\begin{array}{c}23 \\
82.14 \% \\
\end{array}$ & $\begin{array}{c}5 \\
17.86 \% \\
\end{array}$ & 28 \\
\hline Total & 150 & 22 & 172 \\
\hline \multicolumn{4}{|c|}{ Frequency Missing = 1} \\
\hline
\end{tabular}

\begin{tabular}{|l|c|c|c|}
\hline Reported Behaviors & \multicolumn{3}{|c|}{$\begin{array}{l}\text { To Reduce Risk, In The Past 3 Months Or Since The Program, Did You Not Have Sex } \\
\text { After Drinking Or Doing Drugs? }\end{array}$} \\
\hline Survey Period & Did Not Select & Yes (Did Not Have Sex) & Total \\
\hline Baseline & 82 & 13 & 95 \\
\hline 4-Month & $86.32 \%$ & $13.68 \%$ & 49 \\
& 39 & 10 & 28 \\
\hline 12-Month & $79.59 \%$ & $6.41 \%$ & 172 \\
\hline Total & 22 & $21.43 \%$ & 29 \\
\hline Frequency Missing =1 & $78.57 \%$ & & \\
\hline
\end{tabular}




\begin{tabular}{|l|c|c|c|}
\hline Reported Behaviors & \multicolumn{3}{|c|}{ Have You Said No To Sex In The Past 3 Months Or Since The Program? } \\
\hline Survey Period & Did Not Select & Yes & Total \\
\hline \multirow{2}{*}{ Baseline } & 76 & 19 & 95 \\
\hline 4-Month & $80.00 \%$ & $20.00 \%$ & 49 \\
& 41 & 8 & $16.33 \%$ \\
\hline 12-Month & $83.67 \%$ & 4 & 28 \\
\hline Total & 24 & $14.29 \%$ & 172 \\
\hline Frequency Missing = & $85.71 \%$ & 31 & \\
\hline
\end{tabular}

\begin{tabular}{|c|c|c|c|}
\hline \multicolumn{4}{|c|}{ Reported Behaviors } \\
\hline \multirow[t]{2}{*}{ Period } & \multicolumn{3}{|c|}{$\begin{array}{l}\text { In The Past } 3 \text { Months, Have You Used Another Method (Not Listed) To Reduce My } \\
\text { Risk. }\end{array}$} \\
\hline & Did Not Select & Yes & Total \\
\hline Baseline & $\begin{array}{c}93 \\
97.89 \%\end{array}$ & $\begin{array}{c}2 \\
2.11 \%\end{array}$ & 95 \\
\hline 4-Month & $\begin{array}{c}47 \\
95.92 \%\end{array}$ & $\begin{array}{c}2 \\
4.08 \%\end{array}$ & 49 \\
\hline 12-Month & $\begin{array}{c}22 \\
78.57 \%\end{array}$ & $\begin{array}{c}6 \\
21.43 \%\end{array}$ & 28 \\
\hline Total & 162 & 10 & 172 \\
\hline
\end{tabular}

\begin{tabular}{|l|c|c|c|}
\hline Reported Behaviors & \multicolumn{3}{|c|}{ In The Past 3 Months, Have You Had More Than One Partner. } \\
\hline Survey Period & Did Not Select & Yes & Total \\
\hline \multirow{2}{*}{ Baseline } & 87 & 8 & 95 \\
\hline 4-Month & $91.58 \%$ & $8.42 \%$ & 45 \\
& 37 & 8 & $17.78 \%$ \\
\hline 12-Month & $82.22 \%$ & 4 & 28 \\
& 24 & $14.29 \%$ & 168 \\
\hline Total & $85.71 \%$ & 20 & \\
\hline Frequency Missing $\mathbf{5}$ & 148 & & \\
\hline
\end{tabular}

\begin{tabular}{|l|c|c|c|}
\hline Reported Behaviors & \multicolumn{3}{|c|}{$\begin{array}{l}\text { In The Past 3 Months Or Since The Program, I Did Not Use Pills Or Hormonal } \\
\text { Methods For Birth Control. }\end{array}$} \\
\hline Durvey Period & 79 & Yes (Did Not) & Total \\
\hline Baseline & $83.16 \%$ & 16 & 95 \\
\hline 4-Month & 39 & $16.84 \%$ & 45 \\
& $86.67 \%$ & 6 & 28 \\
\hline 12-Month & 22 & 6 & $13.33 \%$ \\
\hline Total & $78.57 \%$ & $21.43 \%$ & 28 \\
\hline Frequency Missing $\mathbf{5}$ & 140 & & \\
\hline
\end{tabular}




\begin{tabular}{|l|c|c|c|}
\hline Reported Behaviors & \multicolumn{3}{|c|}{ In The Past 3 Months Or Since The Program, I Did Not Use Condoms. } \\
\hline Survey Period & Did Not Select & Yes (Did Not) & Total \\
\hline \multirow{2}{*}{ Baseline } & 57 & 38 & 95 \\
\hline 4-Month & $60.00 \%$ & $40.00 \%$ & 45 \\
\hline 12-Month & 29 & 16 & 28 \\
\hline Total & $64.44 \%$ & 12 & \multirow{2}{*}{28} \\
\hline Frequency Missing =5 & 16 & $42.86 \%$ & 168 \\
\hline
\end{tabular}

\begin{tabular}{|c|c|c|c|}
\hline \multicolumn{4}{|c|}{ Reported Behaviors } \\
\hline \multirow[t]{2}{*}{ Survey Period } & \multicolumn{3}{|c|}{$\begin{array}{l}\text { In The Past } 3 \text { Months Or Since The Program, I Only Used The Withdrawal Or } \\
\text { Rhythm Method. }\end{array}$} \\
\hline & Did Not Select & Yes & Total \\
\hline Baseline & $\begin{array}{l}85 \\
89.47\end{array}$ & $\begin{array}{c}10 \\
10.53\end{array}$ & 95 \\
\hline 4-Month & $\begin{array}{c}43 \\
95.56\end{array}$ & $\begin{array}{c}2 \\
4.44\end{array}$ & 45 \\
\hline 12-Month & $\begin{array}{c}22 \\
78.57\end{array}$ & $\begin{array}{c}6 \\
21.43 \\
\end{array}$ & 28 \\
\hline Total & 150 & 18 & 168 \\
\hline
\end{tabular}

\begin{tabular}{|l|c|c|c|}
\hline Reported Behaviors & \multicolumn{3}{|c|}{$\begin{array}{l}\text { In The Past 3 Months Or Since The Program, I Have Had Sex After Drinking Alcohol } \\
\text { Or Using Drugs. }\end{array}$} \\
\hline Period & Did Not Select & Yes & Total \\
\hline Baseline & 79 & 16 & 95 \\
\hline 4-Month & 83.16 & 16.84 & 45 \\
& 33 & 26.67 & 28 \\
\hline 12-Month & 73.33 & 9 & 32.14 \\
\hline Total & 19 & 37 & 168 \\
\hline Frequency Missing $\mathbf{5}$ & 67.86 & & \\
\hline
\end{tabular}

\begin{tabular}{|c|c|c|c|}
\hline \multicolumn{4}{|c|}{ Reported Behaviors } \\
\hline \multirow[t]{2}{*}{ Survey Period } & \multicolumn{3}{|c|}{$\begin{array}{l}\text { In The Past } 3 \text { Months Or Since The Program, I Have Used No Method Of Protection } \\
\text { When Having Sex. }\end{array}$} \\
\hline & Did Not Select & Yes (No Method Used) & Total \\
\hline Baseline & $\begin{array}{c}81 \\
85.26 \%\end{array}$ & $\begin{array}{c}14 \\
14.74 \%\end{array}$ & 95 \\
\hline 4-Month & $\begin{array}{c}39 \\
86.67 \%\end{array}$ & $\begin{array}{c}6 \\
13.33 \%\end{array}$ & 45 \\
\hline 12-Month & $\begin{array}{c}22 \\
78.57 \%\end{array}$ & $\begin{array}{c}6 \\
21.43 \%\end{array}$ & 28 \\
\hline Total & 142 & 26 & 168 \\
\hline
\end{tabular}




\begin{tabular}{|l|c|c|c|}
\hline Reported Behaviors & \multicolumn{3}{|c|}{ In The Past Three Months Or Since The Program, I Have Not Had Sex. } \\
\hline Survey Period & Did Not Select & Yes (Have Not Had Sex) & Total \\
\hline Baseline & 67 & 28 & 95 \\
\hline 4-Month & $70.53 \%$ & $29.47 \%$ & 45 \\
\hline 12-Month & 33 & $26.67 \%$ & 28 \\
\hline Total & $73.33 \%$ & 6 & 12 \\
\hline Frequency Missing $\mathbf{5}$ & 22 & $21.43 \%$ & 268 \\
\hline
\end{tabular}

\begin{tabular}{|l|c|c|c|}
\hline Reported Behaviors & \multicolumn{3}{|c|}{ I Did Not Use A Method Of Protection The Last Time I Had Sex. } \\
\hline Survey Period & Did Not Select & Yes (Did Not Use) & Total \\
\hline Baseline & 79 & 16 & 95 \\
\hline 4-Month & $83.16 \%$ & 0 & 0 \\
\hline 12-Month & 0 & 3 & 28 \\
\hline Total & 25 & $10.71 \%$ & 12 \\
\hline Frequency Missing $\mathbf{5 0}$ & $89.29 \%$ & 19 & 23 \\
\hline
\end{tabular}

\begin{tabular}{|l|c|c|c|}
\hline Reported Behaviors & \multicolumn{3}{|c|}{} \\
\hline Survey Period & Did You Use Pills The Last Time You Had Sex? & Yes & Total \\
\hline \multirow{2}{*}{ Baseline } & No & 26 & 95 \\
\hline \multirow{2}{*}{ 4-Month } & $72.63 \%$ & $27.37 \%$ & 0 \\
\hline
\end{tabular}

\begin{tabular}{|l|c|c|c|}
\hline Reported Behaviors & \multicolumn{3}{|c|}{ Did You Only Use Condoms The Last Time You Had Sex? } \\
\hline Survey Period & No & Yes & Total \\
\hline \multirow{2}{*}{ Baseline } & 60 & 35 & 95 \\
\hline \multirow{2}{*}{ 4-Month } & $63.16 \%$ & $36.84 \%$ & 0 \\
\hline 12-Month & 0 & 0 & 28 \\
\hline Total & 21 & 7 & $25.00 \%$ \\
\hline Frequency Missing $\mathbf{5 0}$ & $75.00 \%$ & 42 & 123 \\
\hline
\end{tabular}

\begin{tabular}{|l|c|c|c|}
\hline Reported Behaviors & \multicolumn{3}{|c|}{} \\
\hline Survey Period & Did You Only Use Spermicide The Last Time You Had Sex? & Yes & 28 \\
\hline 12-Month & 21 & 7 & 123 \\
\hline Total & $75.00 \%$ & $25.00 \%$ & 33 \\
\hline Frequency Missing $=\mathbf{5 0}$ & 90 & \multicolumn{2}{c}{} \\
\hline
\end{tabular}




\begin{tabular}{|l|c|c|c|}
\hline Reported Behaviors & \multicolumn{3}{|c|}{$\begin{array}{l}\text { In The Past Three Months Or Since The Program, I Have Had "Other" Risky } \\
\text { Behaviors (Which Were Not Asked About). }\end{array}$} \\
\hline Survey Period & No & Yes & Total \\
\hline Baseline & 89 & 6 & 95 \\
\hline 4-Month & $93.68 \%$ & $6.32 \%$ & 45 \\
& 45 & 0 & $28 \%$ \\
\hline 12-Month & $100.00 \%$ & 3 & $10.71 \%$ \\
\hline Total & 25 & 9 & 168 \\
\hline Frequency Missing $\mathbf{5}$ & $89.29 \%$ & & \\
\hline
\end{tabular}

\begin{tabular}{|l|c|c|c|}
\hline Reported Behaviors & \multicolumn{3}{|c|}{ Did You Use Depo-Provera/ Implanon The Last Time You Had Sex? } \\
\hline Survey Period & No & Yes & Total \\
\hline Baseline & $\mathbf{8 4}$ & $\mathbf{1 1}$ & $\mathbf{9 5}$ \\
\hline 4-Month & $\mathbf{8 8 . 4 2 \%}$ & $\mathbf{1 1 . 5 8 \%}$ & $\mathbf{0}$ \\
& $\mathbf{0}$ & $\mathbf{0}$ & $\mathbf{2 8}$ \\
\hline 12-Month & $\mathbf{2 8}$ & $\mathbf{0}$ & $\mathbf{0 . 0 0 \%}$ \\
& $\mathbf{1 0 0 . 0 0 \%}$ & $\mathbf{1 1}$ & $\mathbf{1 2 3}$ \\
\hline Total & $\mathbf{1 1 2}$ & & \\
\hline Frequency Missing $\mathbf{5 0}$ & & & \\
\hline
\end{tabular}

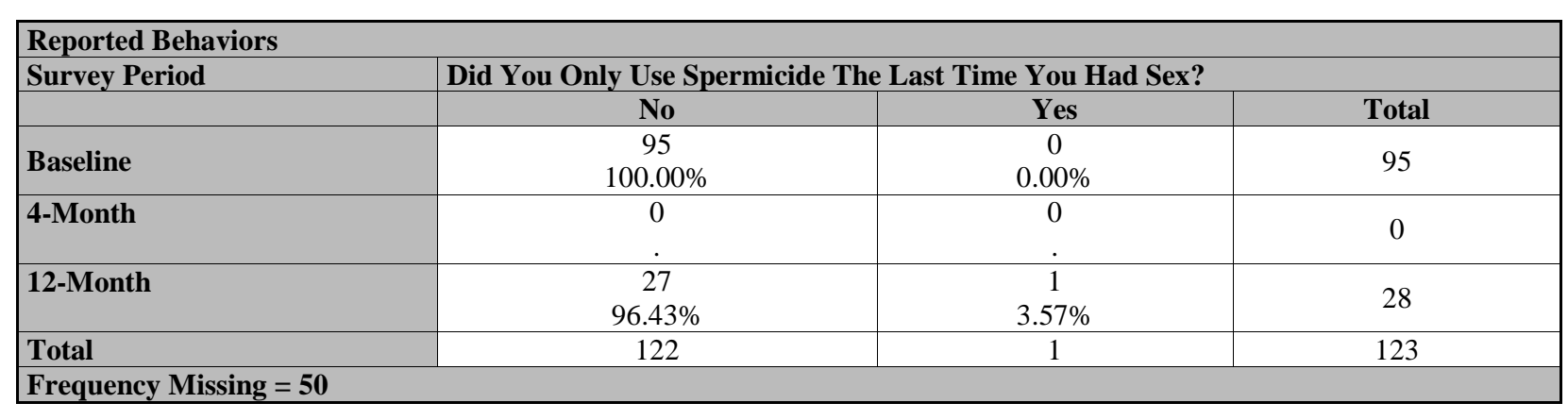

\begin{tabular}{|l|c|c|c|}
\hline Reported Behaviors & \multicolumn{3}{|c|}{ Did You Only Use The Withdrawal/Rhythm Method The Last Time You Had Sex? } \\
\hline Survey Period & No & Yes & Total \\
\hline \multirow{2}{*}{ Baseline } & 76 & 19 & 95 \\
\hline \multirow{2}{*}{ 4-Month } & 80.00 & 20.00 & 0 \\
\hline \multirow{2}{*}{ 12-Month } & 0 & 0 & 28 \\
\hline Total & 17 & 11 & 23 \\
\hline Frequency Missing $=\mathbf{5 0}$ & 60.71 & 39.29 & 123 \\
\hline
\end{tabular}




\section{APPENDIX C: OPINIONS}

\begin{tabular}{|c|c|c|c|c|c|c|}
\hline \multicolumn{7}{|l|}{ Opinions } \\
\hline \multirow[t]{2}{*}{ Survey Period } & \multicolumn{6}{|c|}{$\begin{array}{l}\text { Talking To My Sexual Partner About Using A Method Of Birth Control Before Having Sex } \\
\text { Would Be: }\end{array}$} \\
\hline & Very Good & Somewhat Good & Neutral & $\begin{array}{l}\text { Somewhat } \\
\text { Bad }\end{array}$ & Very Bad & Total \\
\hline Baseline & $\begin{array}{c}81 \\
84.38 \%\end{array}$ & $\begin{array}{c}9 \\
9.38 \%\end{array}$ & $\begin{array}{c}4 \\
4.17 \%\end{array}$ & $\begin{array}{c}1 \\
1.04 \%\end{array}$ & $\begin{array}{c}1 \\
1.04\end{array}$ & 96 \\
\hline 12-Month & $\begin{array}{c}23 \\
82.14 \%\end{array}$ & $\begin{array}{c}4 \\
14.29 \%\end{array}$ & $\begin{array}{c}1 \\
3.57 \%\end{array}$ & $0.00 \%$ & $\begin{array}{c}0 \\
\mathbf{0 . 0 0}\end{array}$ & 28 \\
\hline Total & 104 & 13 & 5 & 1 & 1 & 124 \\
\hline
\end{tabular}

\begin{tabular}{|c|c|c|c|c|c|}
\hline \multicolumn{6}{|l|}{ Opinions } \\
\hline \multirow[t]{2}{*}{ Survey Period } & \multicolumn{5}{|c|}{ Using Birth Control To Prevent Pregnancy Before Sex Would Be: } \\
\hline & Very Good & Somewhat Good & Neutral & Very Bad & Total \\
\hline Baseline & $\begin{array}{c}87 \\
90.63 \% \\
\end{array}$ & $\begin{array}{c}7 \\
7.29 \% \\
\end{array}$ & $\begin{array}{c}0 \\
0.00 \% \\
\end{array}$ & $\begin{array}{c}2 \\
2.08 \% \\
\end{array}$ & 96 \\
\hline 12-Month & $\begin{array}{c}23 \\
85.19 \% \\
\end{array}$ & $\begin{array}{c}1 \\
3.70 \% \\
\end{array}$ & $\begin{array}{c}1 \\
3.70 \% \\
\end{array}$ & $\begin{array}{c}2 \\
7.41 \%\end{array}$ & 27 \\
\hline Total & 110 & 8 & 1 & 4 & 123 \\
\hline
\end{tabular}

\begin{tabular}{|c|c|c|c|c|}
\hline \multicolumn{5}{|l|}{ Opinions } \\
\hline \multirow{2}{*}{ Survey Period } & \multicolumn{4}{|c|}{ Talking To My Partner About Using Condoms Before Having Sex Would Be: } \\
\hline & Very Good & Somewhat Good & Neutral & Total \\
\hline Baseline & $\begin{array}{c}78 \\
81.25 \%\end{array}$ & $\begin{array}{c}8 \\
8.33 \%\end{array}$ & $\begin{array}{c}10 \\
10.42 \%\end{array}$ & 96 \\
\hline 12-Month & $\begin{array}{c}20 \\
74.07 \%\end{array}$ & $\begin{array}{c}4 \\
14.81 \%\end{array}$ & $\begin{array}{c}3 \\
11.11 \%\end{array}$ & 27 \\
\hline Total & 98 & 12 & 13 & 123 \\
\hline
\end{tabular}

\begin{tabular}{|c|c|c|c|c|c|c|}
\hline \multicolumn{7}{|l|}{ Opinions } \\
\hline \multirow{2}{*}{$\begin{array}{l}\text { Survey } \\
\text { Period }\end{array}$} & \multicolumn{6}{|c|}{ Getting latex condoms before having sex would be: } \\
\hline & Very Good & Somewhat Good & Neutral & $\begin{array}{c}\text { Somewhat } \\
\text { Bad }\end{array}$ & Very Bad & Total \\
\hline Baseline & $\begin{array}{c}73 \\
76.04 \%\end{array}$ & $\begin{array}{c}11 \\
11.46 \%\end{array}$ & $\begin{array}{c}11 \\
11.46 \%\end{array}$ & $\begin{array}{c}1 \\
1.04 \%\end{array}$ & $\begin{array}{c}0 \\
0.00 \%\end{array}$ & 96 \\
\hline 12-Month & $\begin{array}{c}23 \\
85.19 \% \\
\end{array}$ & $\begin{array}{c}1 \\
3.70 \%\end{array}$ & $\begin{array}{c}2 \\
7.41 \%\end{array}$ & $\begin{array}{c}0 \\
0.00 \%\end{array}$ & $\begin{array}{c}1 \\
3.70 \%\end{array}$ & 27 \\
\hline Total & 96 & 12 & 13 & 1 & 1 & 123 \\
\hline \multicolumn{7}{|c|}{ Frequency Missing = 1} \\
\hline
\end{tabular}

\begin{tabular}{|l|c|c|c|c|c|}
\hline Opinions & \multicolumn{1}{|c|}{ If I Engage In Unsafe Sex, Going To My Health Care Provider Or Another Clinic To Be } \\
Checked For STIs Would Be: & Somewhat \\
Survey Period & Very Good & Somewhat Good & Neutral & $\begin{array}{c}\text { Total } \\
\text { Bad }\end{array}$ \\
\hline Baseline & 89 & 7 & 0 & 0 & 96 \\
\hline 12-Month & $92.71 \%$ & $7.29 \%$ & $0.00 \%$ & $0.00 \%$ & 1 \\
\hline Total & 26 & 0 & 1 & $3.57 \%$ & 28 \\
\hline
\end{tabular}




\begin{tabular}{|c|c|c|c|c|c|c|}
\hline \multicolumn{7}{|l|}{ Opinions } \\
\hline \multirow[t]{2}{*}{ Survey Period } & \multicolumn{6}{|c|}{$\begin{array}{l}\text { Chances For A Girl In My FOCUS Group To Have An Unplanned Pregnancy In } 6 \text { Months } \\
\text { After Program Are: }\end{array}$} \\
\hline & No Chance & $\begin{array}{l}\text { Very Low } \\
\text { Chance }\end{array}$ & Low Chance & $\begin{array}{l}\text { Moderate } \\
\text { Chance }\end{array}$ & $\begin{array}{c}\text { High } \\
\text { Chance }\end{array}$ & Total \\
\hline Baseline & $\begin{array}{c}1 \\
1.04 \% \\
\end{array}$ & $\begin{array}{c}20 \\
20.83 \% \\
\end{array}$ & $\begin{array}{c}38 \\
39.58 \% \\
\end{array}$ & $\begin{array}{c}27 \\
28.13 \% \\
\end{array}$ & $\begin{array}{c}10 \\
10.42 \% \\
\end{array}$ & 96 \\
\hline 12-Month & $\begin{array}{c}1 \\
3.57 \% \\
\end{array}$ & $\begin{array}{c}3 \\
10.71 \% \\
\end{array}$ & $\begin{array}{c}8 \\
28.57 \% \\
\end{array}$ & $\begin{array}{c}12 \\
42.86 \% \\
\end{array}$ & $\begin{array}{c}4 \\
14.29 \% \\
\end{array}$ & 28 \\
\hline Total & 2 & 23 & 46 & 39 & 14 & 124 \\
\hline
\end{tabular}

\begin{tabular}{|c|c|c|c|c|c|c|}
\hline \multicolumn{7}{|l|}{ Opinions } \\
\hline \multirow[t]{2}{*}{ Survey Period } & \multicolumn{6}{|c|}{$\begin{array}{l}\text { Chances For A Girl In My FOCUS Group To Get An STD During The First } 6 \text { Months After } \\
\text { The Program Are: }\end{array}$} \\
\hline & No Chance & $\begin{array}{l}\text { Very Low } \\
\text { Chance }\end{array}$ & Low Chance & $\begin{array}{l}\text { Moderate } \\
\text { Chance }\end{array}$ & $\begin{array}{c}\text { High } \\
\text { Chance }\end{array}$ & Total \\
\hline Baseline & $\begin{array}{c}8 \\
8.33 \%\end{array}$ & $33.33 \%$ & $\begin{array}{c}30 \\
31.25 \%\end{array}$ & $\begin{array}{c}24 \\
25.00 \%\end{array}$ & $2.08 \%$ & 96 \\
\hline 12-Month & $\begin{array}{c}3 \\
10.71 \%\end{array}$ & $\begin{array}{c}2 \\
7.14 \%\end{array}$ & $\begin{array}{c}11 \\
39.29 \%\end{array}$ & $\begin{array}{c}9 \\
32.14 \%\end{array}$ & $\begin{array}{c}3 \\
10.71 \%\end{array}$ & 28 \\
\hline Total & 11 & 34 & 41 & 33 & 5 & 124 \\
\hline
\end{tabular}

\section{APPENDIX D: PERCEIVED OPINIONS OF FRIENDS}

\begin{tabular}{|l|l|c|c|c|c|c|}
\hline Perceived Opinions of Friends \\
\hline Survey Period & \multicolumn{7}{l|}{$\begin{array}{l}\text { My Friends Think It Is Important For Me To Talk To My Partner About Using Birth } \\
\text { Control. } \\
\text { Very True }\end{array}$} & $\begin{array}{c}\text { Somewhat } \\
\text { True }\end{array}$ & Neutral & $\begin{array}{c}\text { Somewhat } \\
\text { Untrue }\end{array}$ & Very Untrue & Total \\
\hline Baseline & 58 & 19 & 16 & 1 & 2 & 96 \\
\hline 12-Month & $60.42 \%$ & $19.79 \%$ & $16.67 \%$ & $1.04 \%$ & $2.08 \%$ & 96 \\
\hline Total & 17 & 2 & 8 & 0 & 1 & 28 \\
\hline
\end{tabular}

\begin{tabular}{|l|l|c|c|c|c|c|}
\hline Perceived Opinions of Friends \\
\hline Survey Period & $\begin{array}{l}\text { My Friends Think It Is Important For Me To Go To A Healthcare Provider To Discuss } \\
\text { Choosing Birth Control. }\end{array}$ \\
\hline & Very True & $\begin{array}{c}\text { Somewhat } \\
\text { True }\end{array}$ & Neutral & $\begin{array}{c}\text { Somewhat } \\
\text { Untrue }\end{array}$ & Very Untrue & Total \\
\hline Baseline & 41 & 14 & 26 & 8 & 7 & 96 \\
\hline 12-Month & $42.71 \%$ & $14.58 \%$ & $27.08 \%$ & $8.33 \%$ & $7.29 \%$ & 0 \\
\hline Total & 15 & 2 & 9 & 2 & $0.00 \%$ & 28 \\
\hline
\end{tabular}

\begin{tabular}{|c|c|c|c|c|c|c|}
\hline \multicolumn{7}{|c|}{ Perceived Opinions of Friends } \\
\hline \multirow[t]{2}{*}{ Survey Period } & \multicolumn{6}{|c|}{ My Friends Think It Is Important For Me To Talk To My Partner About Using Condoms. } \\
\hline & Very True & $\begin{array}{c}\text { Somewhat } \\
\text { True }\end{array}$ & Neutral & $\begin{array}{c}\text { Somewhat } \\
\text { Untrue }\end{array}$ & Very Untrue & Total \\
\hline Baseline & $\begin{array}{c}48 \\
50.00 \%\end{array}$ & $\begin{array}{c}21 \\
21.88 \%\end{array}$ & $\begin{array}{c}21 \\
21.88 \%\end{array}$ & $\begin{array}{c}4 \\
4.17 \%\end{array}$ & $\begin{array}{c}2 \\
2.08 \%\end{array}$ & 96 \\
\hline 12-Month & $\begin{array}{c}15 \\
55.56 \%\end{array}$ & $\begin{array}{c}3 \\
11.11 \%\end{array}$ & $\begin{array}{c}8 \\
29.63 \%\end{array}$ & $\begin{array}{c}0 \\
0.00 \%\end{array}$ & $\begin{array}{c}1 \\
3.70 \%\end{array}$ & 27 \\
\hline Total & 63 & 24 & 29 & 4 & 3 & 123 \\
\hline
\end{tabular}




\begin{tabular}{|c|c|c|c|c|c|c|}
\hline \multicolumn{7}{|c|}{ Perceived Opinions of Friends } \\
\hline \multirow[t]{2}{*}{ Survey Period } & \multicolumn{6}{|c|}{ My Friends Think It Is Important For Me To Get Condoms Before Having Sex. } \\
\hline & Very True & $\begin{array}{c}\text { Somewhat } \\
\text { True }\end{array}$ & Neutral & $\begin{array}{c}\text { Somewhat } \\
\text { Untrue }\end{array}$ & Very Untrue & Total \\
\hline Baseline & $\begin{array}{c}44 \\
45.83 \% \\
\end{array}$ & $\begin{array}{c}22 \\
22.92 \%\end{array}$ & $\begin{array}{c}24 \\
25.00 \%\end{array}$ & $\begin{array}{c}4 \\
4.17 \%\end{array}$ & $\begin{array}{c}2 \\
2.08 \%\end{array}$ & 96 \\
\hline 12-Month & $\begin{array}{c}14 \\
50.00 \%\end{array}$ & $\begin{array}{c}5 \\
17.86 \%\end{array}$ & $\begin{array}{c}7 \\
25.00 \% \\
\end{array}$ & $\begin{array}{c}1 \\
3.57 \%\end{array}$ & $\begin{array}{c}1 \\
3.57 \% \\
\end{array}$ & 28 \\
\hline Total & 58 & 27 & 31 & 5 & 3 & 124 \\
\hline
\end{tabular}

\begin{tabular}{|c|c|c|c|c|c|c|}
\hline \multicolumn{7}{|c|}{ Perceived Opinions of Friends } \\
\hline \multirow[t]{2}{*}{ Survey Period } & \multicolumn{6}{|c|}{ My Friends Think It Is Important For Me To Use Condoms Consistently And Correctly. } \\
\hline & Very True & $\begin{array}{c}\text { Somewhat } \\
\text { True }\end{array}$ & Neutral & $\begin{array}{l}\text { Somewhat } \\
\text { Untrue }\end{array}$ & Very Untrue & Total \\
\hline Baseline & $\begin{array}{c}53 \\
55.21 \%\end{array}$ & $\begin{array}{c}14 \\
14.58 \%\end{array}$ & $\begin{array}{c}25 \\
26.04 \%\end{array}$ & $\begin{array}{c}2 \\
2.08 \%\end{array}$ & $\begin{array}{c}2 \\
2.08 \%\end{array}$ & 96 \\
\hline 12-Month & $\begin{array}{c}18 \\
66.67 \% \\
\end{array}$ & $\begin{array}{c}1 \\
3.70 \%\end{array}$ & $\begin{array}{c}6 \\
22.22 \% \\
\end{array}$ & $\begin{array}{c}0 \\
0.00 \%\end{array}$ & $\begin{array}{c}2 \\
7.41 \%\end{array}$ & 27 \\
\hline Total & 71 & 15 & 31 & 2 & 4 & 123 \\
\hline
\end{tabular}

\section{APPENDIX E: INTENTIONS FOR FUTURE PRACTICE}

\begin{tabular}{|c|c|c|c|c|c|c|}
\hline \multicolumn{7}{|c|}{ Intentions for Future Practice } \\
\hline \multirow[t]{2}{*}{ Survey Period } & \multicolumn{6}{|c|}{ I Plan To Talk To My Partner About Using Birth Control Before Sex. } \\
\hline & Very True & $\begin{array}{c}\text { Somewhat } \\
\text { True }\end{array}$ & Neutral & $\begin{array}{c}\text { Somewhat } \\
\text { Untrue }\end{array}$ & Very Untrue & Total \\
\hline Baseline & $\begin{array}{c}71 \\
73.96 \%\end{array}$ & $\begin{array}{c}14 \\
14.58 \%\end{array}$ & $\begin{array}{c}7 \\
7.29 \%\end{array}$ & $\begin{array}{c}\mathbf{3} \\
\mathbf{3 . 1 3} \%\end{array}$ & $\begin{array}{c}1 \\
1.04 \%\end{array}$ & 96 \\
\hline 12-Month & $\begin{array}{c}21 \\
75.00 \%\end{array}$ & $\begin{array}{c}5 \\
17.86 \%\end{array}$ & $\begin{array}{c}2 \\
7.14 \%\end{array}$ & $\begin{array}{c}0 \\
0.00\end{array}$ & $\begin{array}{c}0 \\
0.00\end{array}$ & 28 \\
\hline Total & 92 & 19 & 9 & 3 & 1 & 124 \\
\hline
\end{tabular}

\begin{tabular}{|c|c|c|c|c|c|c|}
\hline \multicolumn{7}{|c|}{ Intentions for Future Practice } \\
\hline \multirow[t]{2}{*}{ Survey Period } & \multicolumn{6}{|c|}{ I Plan To Use A Birth Control To Prevent Pregnancy Before Having Sex. } \\
\hline & Very True & $\begin{array}{c}\text { Somewhat } \\
\text { True }\end{array}$ & Neutral & $\begin{array}{l}\text { Somewhat } \\
\text { Untrue }\end{array}$ & Very Untrue & Total \\
\hline Baseline & $\begin{array}{c}81 \\
84.38 \%\end{array}$ & $\begin{array}{c}6 \\
6.25 \%\end{array}$ & $\begin{array}{c}6 \\
6.25 \%\end{array}$ & $\begin{array}{c}1 \\
1.04 \%\end{array}$ & $\begin{array}{c}2 \\
2.08 \%\end{array}$ & 96 \\
\hline 12-Month & $\begin{array}{c}21 \\
77.78 \%\end{array}$ & $\begin{array}{c}1 \\
3.70 \%\end{array}$ & $\begin{array}{c}3 \\
11.11 \%\end{array}$ & $\begin{array}{c}1 \\
3.70 \%\end{array}$ & $\begin{array}{c}1 \\
3.70 \%\end{array}$ & 27 \\
\hline Total & 102 & 7 & 9 & 2 & 3 & 123 \\
\hline
\end{tabular}

\begin{tabular}{|c|c|c|c|c|c|c|}
\hline \multicolumn{7}{|c|}{ Intentions for Future Practice } \\
\hline \multirow{2}{*}{ Survey Period } & \multicolumn{6}{|c|}{ I Plan To Talk To Partner About Using A Condom Before Having Sex. } \\
\hline & Very True & $\begin{array}{c}\text { Somewhat } \\
\text { True }\end{array}$ & Neutral & $\begin{array}{c}\text { Somewhat } \\
\text { Untrue }\end{array}$ & Very Untrue & Total \\
\hline Baseline & $\begin{array}{c}64 \\
66.67 \%\end{array}$ & $\begin{array}{c}13 \\
13.54 \%\end{array}$ & $\begin{array}{c}7 \\
7.29 \%\end{array}$ & $\begin{array}{c}6 \\
6.25 \%\end{array}$ & $\begin{array}{c}6 \\
6.25 \% \\
\end{array}$ & 96 \\
\hline 12-Month & $\begin{array}{c}16 \\
59.26 \%\end{array}$ & $\begin{array}{c}7 \\
25.93 \% \\
\end{array}$ & $\begin{array}{c}2 \\
7.41 \%\end{array}$ & $\begin{array}{c}0 \\
0.00\end{array}$ & $\begin{array}{c}2 \\
7.41 \%\end{array}$ & 27 \\
\hline Total & 80 & 20 & 9 & 6 & 8 & 123 \\
\hline
\end{tabular}




\begin{tabular}{|c|c|c|c|c|c|c|}
\hline \multicolumn{7}{|c|}{ Intentions for Future Practice } \\
\hline \multirow{2}{*}{ Survey Period } & \multicolumn{6}{|c|}{ I Plan To Get Condoms Before Having Sex. } \\
\hline & Very True & $\begin{array}{c}\text { Somewhat } \\
\text { True }\end{array}$ & Neutral & $\begin{array}{c}\text { Somewhat } \\
\text { Untrue }\end{array}$ & Very Untrue & Total \\
\hline Baseline & $\begin{array}{c}63 \\
65.63 \%\end{array}$ & $\begin{array}{c}16 \\
16.67 \%\end{array}$ & $\begin{array}{c}7 \\
7.29 \%\end{array}$ & $\begin{array}{c}2 \\
2.08 \%\end{array}$ & $\begin{array}{c}8 \\
8.33 \%\end{array}$ & 96 \\
\hline 12-Month & $\begin{array}{c}16 \\
57.14 \%\end{array}$ & $\begin{array}{c}4 \\
14.29 \%\end{array}$ & $\begin{array}{c}3 \\
10.71 \%\end{array}$ & $\begin{array}{c}3 \\
10.71 \%\end{array}$ & $\begin{array}{c}2 \\
7.14 \%\end{array}$ & 28 \\
\hline Total & 79 & 20 & 10 & 5 & 10 & 124 \\
\hline
\end{tabular}

\begin{tabular}{|c|c|c|c|c|c|c|}
\hline \multicolumn{7}{|c|}{ Intentions for Future Practice } \\
\hline \multirow[t]{2}{*}{ Period } & \multicolumn{6}{|c|}{ I Plan To Use Condom Consistently And Correctly When Having Sex. } \\
\hline & Very True & $\begin{array}{c}\text { Somewhat } \\
\text { True }\end{array}$ & Neutral & $\begin{array}{c}\text { Somewhat } \\
\text { Untrue }\end{array}$ & Very Untrue & Total \\
\hline Baseline & $\begin{array}{c}65 \\
68.42 \% \\
\end{array}$ & $\begin{array}{c}11 \\
11.58 \%\end{array}$ & $\begin{array}{c}6 \\
6.32 \%\end{array}$ & $\begin{array}{c}7 \\
7.37 \%\end{array}$ & $\begin{array}{c}6 \\
6.32 \%\end{array}$ & 95 \\
\hline 12-Month & $\begin{array}{c}17 \\
60.71 \%\end{array}$ & $\begin{array}{c}3 \\
10.71 \%\end{array}$ & $\begin{array}{c}3 \\
10.71 \%\end{array}$ & $\begin{array}{c}3 \\
10.71 \%\end{array}$ & $\begin{array}{c}2 \\
7.14 \%\end{array}$ & 28 \\
\hline Total & 82 & 14 & 9 & 10 & 8 & 123 \\
\hline
\end{tabular}

\begin{tabular}{|c|c|c|c|c|c|c|}
\hline \multicolumn{7}{|c|}{ Intentions for Future Practice } \\
\hline \multirow{2}{*}{ Survey Period } & \multicolumn{6}{|c|}{ I Plan To Be Checked For STD Infections If Engaged In Unsafe Sex. } \\
\hline & Very True & $\begin{array}{c}\text { Somewhat } \\
\text { True }\end{array}$ & Neutral & $\begin{array}{c}\text { Somewhat } \\
\text { Untrue }\end{array}$ & Very Untrue & Total \\
\hline Baseline & $\begin{array}{c}76 \\
79.17 \%\end{array}$ & $\begin{array}{c}14 \\
14.58 \%\end{array}$ & $\begin{array}{c}1 \\
1.04 \%\end{array}$ & $\begin{array}{c}2 \\
2.08 \%\end{array}$ & $\begin{array}{c}3 \\
3.13 \%\end{array}$ & 96 \\
\hline 12-Month & $\begin{array}{c}21 \\
75.00 \%\end{array}$ & $\begin{array}{c}5 \\
17.86 \%\end{array}$ & $\begin{array}{c}2 \\
7.14 \%\end{array}$ & $\begin{array}{c}\mathbf{0} \\
\mathbf{0 . 0 0}\end{array}$ & $\begin{array}{c}\mathbf{0} \\
0.00\end{array}$ & 28 \\
\hline Total & 97 & 19 & 3 & 2 & 3 & 124 \\
\hline
\end{tabular}

\section{APPENDIX F：PERCEPTIONS OF RISK}

\begin{tabular}{|c|c|c|c|c|c|c|}
\hline \multicolumn{7}{|c|}{ Perception of Risk } \\
\hline \multirow[t]{2}{*}{ Survey Period } & \multicolumn{6}{|c|}{$\begin{array}{l}\text { Compared To Other Typical Girls In Your Class/Group, Would You Say That Your } \\
\text { Chance Of Having An Unplanned Pregnancy In The First } 6 \text { Months After Participating In } \\
\text { The FOCUS Program Is: }\end{array}$} \\
\hline & Much lower & Lower & About same & Higher & $\begin{array}{c}\text { Much } \\
\text { Higher }\end{array}$ & Total \\
\hline Baseline & $\begin{array}{c}44 \\
46.32 \% \\
\end{array}$ & $\begin{array}{c}30 \\
31.58 \%\end{array}$ & $\begin{array}{c}20 \\
21.05 \%\end{array}$ & $\begin{array}{c}1 \\
1.05 \%\end{array}$ & $\begin{array}{c}0 \\
0.00 \%\end{array}$ & 95 \\
\hline 12-Month & $\begin{array}{c}15 \\
53.57 \%\end{array}$ & $\begin{array}{c}6 \\
21.43 \%\end{array}$ & $\begin{array}{c}5 \\
17.86 \%\end{array}$ & $\begin{array}{c}1 \\
3.57 \%\end{array}$ & $\begin{array}{c}1 \\
3.57 \%\end{array}$ & 28 \\
\hline Total & 59 & 36 & 25 & 2 & 1 & 123 \\
\hline
\end{tabular}

\begin{tabular}{|c|c|c|c|c|c|}
\hline \multicolumn{6}{|c|}{ Perception of Risk } \\
\hline \multirow[t]{2}{*}{ Survey Period } & \multicolumn{5}{|c|}{$\begin{array}{l}\text { With Regard To Your Chances Of Having An Unplanned Pregnancy, Would You Say Your } \\
\text { Sexual Behavior Is: }\end{array}$} \\
\hline & Very Safe & Fairly Safe & Somewhat Risky & Very Risky & Total \\
\hline Baseline & $\begin{array}{c}46 \\
47.92 \%\end{array}$ & $\begin{array}{c}26 \\
27.08 \%\end{array}$ & $\begin{array}{c}16 \\
16.67 \%\end{array}$ & $\begin{array}{c}8 \\
8.33 \%\end{array}$ & 96 \\
\hline 12-Month & $\begin{array}{c}15 \\
53.57 \%\end{array}$ & $\begin{array}{c}6 \\
21.43 \% \\
\end{array}$ & $\begin{array}{c}5 \\
17.86 \% \\
\end{array}$ & $\begin{array}{c}2 \\
7.14 \%\end{array}$ & 28 \\
\hline Total & 61 & 32 & 21 & 10 & 124 \\
\hline
\end{tabular}




\begin{tabular}{|c|c|c|c|c|c|}
\hline \multicolumn{6}{|c|}{ Perception of Risk } \\
\hline \multirow[t]{2}{*}{ Survey Period } & \multicolumn{5}{|c|}{$\begin{array}{l}\text { What Do You Think Your Chances Are Of Getting An STI In The First } 6 \text { Months After } \\
\text { Participating In The FOCUS Program? }\end{array}$} \\
\hline & No Chance & Very Low Chance & Low Chance & $\begin{array}{l}\text { Moderate } \\
\text { Chance }\end{array}$ & Total \\
\hline Baseline & $\begin{array}{c}63 \\
65.63 \% \\
\end{array}$ & $\begin{array}{c}25 \\
26.04 \% \\
\end{array}$ & $\begin{array}{c}7 \\
7.29 \%\end{array}$ & $\begin{array}{c}1 \\
1.04 \%\end{array}$ & 96 \\
\hline 12-Month & $\begin{array}{c}15 \\
53.57 \% \\
\end{array}$ & $\begin{array}{c}9 \\
32.14 \% \\
\end{array}$ & $\begin{array}{c}3 \\
10.71 \% \\
\end{array}$ & $\begin{array}{c}1 \\
3.57 \%\end{array}$ & 28 \\
\hline Total & 78 & 34 & 10 & 2 & 124 \\
\hline
\end{tabular}

\begin{tabular}{|c|c|c|c|c|c|}
\hline \multicolumn{6}{|c|}{ Perception of Risk } \\
\hline \multirow[t]{2}{*}{ Survey Period } & \multicolumn{5}{|c|}{$\begin{array}{l}\text { Compared To Other Girls In The FOCUS Program, Would You Say That Your Chance Of } \\
\text { Getting An STI During The First } 6 \text { Months After The Focus Program Is: }\end{array}$} \\
\hline & Much lower & Lower & About same & Higher & Total \\
\hline Baseline & $\begin{array}{c}53 \\
55.79 \%\end{array}$ & $\begin{array}{c}26 \\
27.37 \%\end{array}$ & $\begin{array}{c}16 \\
16.84 \%\end{array}$ & $\begin{array}{c}0 \\
0.00 \%\end{array}$ & 95 \\
\hline 12-Month & $\begin{array}{c}18 \\
64.29 \% \\
\end{array}$ & $\begin{array}{c}7 \\
25.00 \% \\
\end{array}$ & $\begin{array}{c}2 \\
7.14 \% \\
\end{array}$ & $\begin{array}{c}1 \\
3.57 \% \\
\end{array}$ & 28 \\
\hline Total & 71 & 33 & 18 & 1 & 123 \\
\hline
\end{tabular}

\begin{tabular}{|l|c|c|c|c|c|}
\hline Perception of Risk & \multicolumn{6}{l|}{$\begin{array}{l}\text { With Regard To Your Chances Of Getting An STI, Would You Say Your Sexual Behavior } \\
\text { Is: }\end{array}$} & Very Safe & Fairly Safe & Somewhat Risky & Very Risky & Total \\
\hline & 61 & 21 & 9 & 5 & 56 \\
\hline \multirow{2}{*}{ Baseline } & $63.54 \%$ & $21.88 \%$ & $9.38 \%$ & 0 & $21 \%$ \\
\hline \multirow{2}{*}{ 12-Month } & 21 & 5 & 2 & $0.00 \%$ & 28 \\
\hline Total & $75.00 \%$ & $17.86 \%$ & $7.14 \%$ & 11 & 124 \\
\hline
\end{tabular}

\begin{tabular}{|l|c|c|c|}
\hline Perception of Risk & Which Of The Following Fits Your Own Thoughts The Best? \\
\hline Survey Period & Need to use condoms more often & Don't Need to Change & Total \\
\hline Baseline & 38 & 58 & 96 \\
\hline 12-Month & $39.58 \%$ & $60.42 \%$ & 26 \\
\hline Total & 7 & 19 & 122 \\
\hline Frequency Missing = & $26.92 \%$ & $73.08 \%$ & 77 \\
\hline
\end{tabular}

\begin{tabular}{|l|c|c|c|}
\hline Perception of Risk & Which Of The Following Fits Your Own Thoughts The Best? & Don't need to change & Total \\
\hline Survey Period & $\begin{array}{c}\text { Need to be more careful about who } \\
\text { becomes my sexual partner }\end{array}$ & 76 & 96 \\
\hline Baseline & 20 & $79.17 \%$ & 21 \\
\hline 12-Month & $20.83 \%$ & $87.50 \%$ & 24 \\
\hline Total & 3 & 97 & 120 \\
\hline Frequency Missing $\mathbf{4}$ & $22.50 \%$ & & \\
\hline
\end{tabular}




\section{APPENDIX G: ATTITUDES REGARDING CONDOM USE}

\begin{tabular}{|c|c|c|c|c|c|c|}
\hline \multicolumn{7}{|c|}{ Attitudes Regarding Condom Use } \\
\hline \multirow[t]{2}{*}{ Survey Period } & \multicolumn{6}{|c|}{ I Don't Like Sex With Condoms. } \\
\hline & Strongly Agree & $\begin{array}{c}\text { Agree } \\
\text { Somewhat }\end{array}$ & Neutral & $\begin{array}{l}\text { Disagree } \\
\text { Somewhat }\end{array}$ & $\begin{array}{l}\text { Strongly } \\
\text { Disagree }\end{array}$ & Total \\
\hline Baseline & $\begin{array}{c}24 \\
25.00 \%\end{array}$ & $\begin{array}{c}20 \\
20.83 \%\end{array}$ & $\begin{array}{c}22 \\
22.92 \%\end{array}$ & $\begin{array}{c}11 \\
11.46 \%\end{array}$ & $\begin{array}{c}19 \\
19.79 \%\end{array}$ & 96 \\
\hline 12-Month & $\begin{array}{c}7 \\
25.00 \%\end{array}$ & $\begin{array}{c}6 \\
21.43 \%\end{array}$ & $\begin{array}{c}6 \\
21.43 \%\end{array}$ & $\begin{array}{c}3 \\
10.71 \%\end{array}$ & $\begin{array}{c}6 \\
21.43 \%\end{array}$ & 28 \\
\hline Total & 31 & 26 & 28 & 14 & 25 & 124 \\
\hline
\end{tabular}

\begin{tabular}{|c|c|c|c|c|c|c|}
\hline \multicolumn{7}{|c|}{ Attitudes Regarding Condom Use } \\
\hline \multirow[t]{2}{*}{ Survey Period } & \multicolumn{6}{|c|}{ Condoms Decrease The Feeling During Sex. } \\
\hline & Strongly Agree & $\begin{array}{c}\text { Agree } \\
\text { Somewhat }\end{array}$ & Neutral & $\begin{array}{c}\text { Disagree } \\
\text { Somewhat }\end{array}$ & $\begin{array}{l}\text { Strongly } \\
\text { Disagree }\end{array}$ & Total \\
\hline Baseline & $\begin{array}{c}15 \\
15.63 \% \\
\end{array}$ & $\begin{array}{c}30 \\
31.25 \%\end{array}$ & $\begin{array}{c}35 \\
36.46 \% \\
\end{array}$ & $\begin{array}{c}8 \\
8.33 \%\end{array}$ & $\begin{array}{c}8 \\
8.33 \%\end{array}$ & 96 \\
\hline 12-Month & $\begin{array}{c}6 \\
21.43 \% \\
\end{array}$ & $\begin{array}{c}9 \\
32.14 \% \\
\end{array}$ & $\begin{array}{c}8 \\
28.57 \% \\
\end{array}$ & $\begin{array}{c}2 \\
7.14 \% \\
\end{array}$ & $\begin{array}{c}3 \\
10.71 \% \\
\end{array}$ & 28 \\
\hline Total & 21 & 39 & 43 & 10 & 11 & 124 \\
\hline
\end{tabular}

\begin{tabular}{|c|c|c|c|c|c|c|}
\hline \multicolumn{7}{|c|}{ Attitudes Regarding Condom Use } \\
\hline \multirow[t]{2}{*}{ Survey Period } & \multicolumn{6}{|c|}{ Having My Partner Put On A Condom Spoils The Mood. } \\
\hline & Strongly Agree & $\begin{array}{c}\text { Agree } \\
\text { Somewhat }\end{array}$ & Neutral & $\begin{array}{c}\text { Disagree } \\
\text { Somewhat }\end{array}$ & $\begin{array}{l}\text { Strongly } \\
\text { Disagree }\end{array}$ & Total \\
\hline Baseline & $\begin{array}{c}11 \\
11.46 \% \\
\end{array}$ & $\begin{array}{c}17 \\
17.71 \% \\
\end{array}$ & $\begin{array}{c}28 \\
29.17 \% \\
\end{array}$ & $\begin{array}{c}15 \\
15.63 \% \\
\end{array}$ & $\begin{array}{c}25 \\
26.04 \% \\
\end{array}$ & 96 \\
\hline 12-Month & $\begin{array}{c}2 \\
7.14 \% \\
\end{array}$ & $\begin{array}{c}3 \\
10.71 \% \\
\end{array}$ & $\begin{array}{c}11 \\
39.29 \% \\
\end{array}$ & $\begin{array}{c}5 \\
17.86 \% \\
\end{array}$ & $\begin{array}{c}7 \\
25.00 \% \\
\end{array}$ & 28 \\
\hline Total & 13 & 20 & 39 & 20 & 32 & 124 \\
\hline
\end{tabular}

Attitudes Regarding Condom Use

\begin{tabular}{|c|c|c|c|c|c|c|}
\hline \multirow[t]{2}{*}{ Survey Period } & \multicolumn{6}{|c|}{ I Enjoy Sex More When My Partner Uses Condoms. } \\
\hline & Strongly Agree & $\begin{array}{c}\text { Agree } \\
\text { Somewhat }\end{array}$ & Neutral & $\begin{array}{l}\text { Disagree } \\
\text { Somewhat }\end{array}$ & $\begin{array}{l}\text { Strongly } \\
\text { Disagree }\end{array}$ & Total \\
\hline Baseline & $\begin{array}{c}11 \\
11.46 \% \\
\end{array}$ & $\begin{array}{c}10 \\
10.42 \%\end{array}$ & $\begin{array}{c}37 \\
38.54 \% \\
\end{array}$ & $\begin{array}{c}24 \\
25.00 \% \\
\end{array}$ & $\begin{array}{c}14 \\
14.58 \% \\
\end{array}$ & 96 \\
\hline 12-Month & $\begin{array}{c}3 \\
10.71 \% \\
\end{array}$ & $\begin{array}{c}2 \\
7.14 \% \\
\end{array}$ & $\begin{array}{c}15 \\
53.57 \% \\
\end{array}$ & $\begin{array}{c}4 \\
14.29 \% \\
\end{array}$ & $\begin{array}{c}4 \\
14.29 \% \\
\end{array}$ & 28 \\
\hline Total & 14 & 12 & 52 & 28 & 18 & 124 \\
\hline
\end{tabular}




\section{APPENDIX H: ATTITUDES RELATED TO ALCOHOL USE}

\begin{tabular}{|c|c|c|c|c|c|c|}
\hline \multicolumn{7}{|c|}{ Attitudes Related to Alcohol Use } \\
\hline \multirow[t]{2}{*}{ Survey Period } & \multicolumn{6}{|c|}{$\begin{array}{l}\text { I Am More Likely To Have Sex With Someone I Do Not Know Very Well After A Few } \\
\text { Drinks. }\end{array}$} \\
\hline & Strongly Agree & $\begin{array}{c}\text { Agree } \\
\text { Somewhat }\end{array}$ & Neutral & $\begin{array}{c}\text { Disagree } \\
\text { Somewhat }\end{array}$ & $\begin{array}{l}\text { Strongly } \\
\text { Disagree }\end{array}$ & Total \\
\hline Baseline & $\begin{array}{c}7 \\
7.29 \%\end{array}$ & $\begin{array}{c}16 \\
16.67 \%\end{array}$ & $\begin{array}{c}10 \\
10.42 \%\end{array}$ & $\begin{array}{c}11 \\
11.46 \%\end{array}$ & $\begin{array}{c}52 \\
54.17 \%\end{array}$ & 96 \\
\hline 12-Month & $\begin{array}{c}3 \\
10.71 \% \\
\end{array}$ & $\begin{array}{c}4 \\
14.29 \% \\
\end{array}$ & $\begin{array}{c}6 \\
21.43 \% \\
\end{array}$ & $\begin{array}{c}4 \\
14.29 \% \\
\end{array}$ & $\begin{array}{c}11 \\
39.29 \% \\
\end{array}$ & 28 \\
\hline Total & 10 & 20 & 16 & 15 & 63 & 124 \\
\hline
\end{tabular}

Attitudes Related to Alcohol Use

\begin{tabular}{|c|c|c|c|c|c|c|}
\hline \multicolumn{7}{|c|}{ Attitudes Related to Alcohol Use } \\
\hline \multirow[t]{2}{*}{ Survey Period } & \multicolumn{6}{|c|}{ I Enjoy Sex More After A Few Drinks. } \\
\hline & Strongly Agree & $\begin{array}{c}\text { Agree } \\
\text { Somewhat }\end{array}$ & Neutral & $\begin{array}{l}\text { Disagree } \\
\text { Somewhat }\end{array}$ & $\begin{array}{l}\text { Strongly } \\
\text { Disagree }\end{array}$ & Total \\
\hline Baseline & $\begin{array}{c}13 \\
13.54 \%\end{array}$ & $\begin{array}{c}18 \\
18.75 \%\end{array}$ & $\begin{array}{c}20 \\
20.83 \%\end{array}$ & $\begin{array}{c}9 \\
9.38 \%\end{array}$ & $\begin{array}{c}36 \\
37.50 \%\end{array}$ & 96 \\
\hline 12-Month & $\begin{array}{c}4 \\
14.29 \%\end{array}$ & $\begin{array}{c}5 \\
17.86 \%\end{array}$ & $\begin{array}{c}11 \\
39.29 \%\end{array}$ & $\begin{array}{c}2 \\
7.14 \%\end{array}$ & $\begin{array}{c}6 \\
21.43 \%\end{array}$ & 28 \\
\hline Total & 17 & 23 & 31 & 11 & 42 & 124 \\
\hline
\end{tabular}

\begin{tabular}{|c|c|c|c|c|c|c|}
\hline \multicolumn{7}{|c|}{ Attitudes Related to Alcohol Use } \\
\hline \multirow[t]{2}{*}{ Survey Period } & \multicolumn{6}{|c|}{ I Am More Likely To Have Sex Without Condoms After A Few Drinks. } \\
\hline & Strongly Agree & $\begin{array}{c}\text { Agree } \\
\text { Somewhat }\end{array}$ & Neutral & $\begin{array}{c}\text { Disagree } \\
\text { Somewhat }\end{array}$ & $\begin{array}{l}\text { Strongly } \\
\text { Disagree }\end{array}$ & Total \\
\hline Baseline & $\begin{array}{c}9 \\
9.38 \% \\
\end{array}$ & $\begin{array}{c}24 \\
25.00 \% \\
\end{array}$ & $\begin{array}{c}20 \\
20.83 \%\end{array}$ & $\begin{array}{c}12 \\
12.50 \%\end{array}$ & $\begin{array}{c}31 \\
32.29 \%\end{array}$ & 96 \\
\hline 12-Month & $\begin{array}{c}3 \\
10.71 \%\end{array}$ & $\begin{array}{c}6 \\
21.43 \% \\
\end{array}$ & $\begin{array}{c}7 \\
25.00 \%\end{array}$ & $\begin{array}{c}3 \\
10.71 \%\end{array}$ & $\begin{array}{c}9 \\
32.14 \%\end{array}$ & 28 \\
\hline Total & 12 & 30 & 27 & 15 & 40 & 124 \\
\hline
\end{tabular}




\section{APPENDIX I: PERCEPTION OF COMFORT RELATED TO HEALTH SEEKING BEHAVIORS}

\begin{tabular}{|c|c|c|c|c|c|c|}
\hline \multicolumn{7}{|c|}{ Perception of Comfort Related to Health Seeking Behaviors } \\
\hline \multirow[t]{2}{*}{ Survey Period } & \multicolumn{6}{|c|}{ How Difficult Is It To Talk To Your Partner About Birth Control Before Having Sex? } \\
\hline & Very Difficult & $\begin{array}{c}\text { Somewhat } \\
\text { Difficult }\end{array}$ & Neutral & $\begin{array}{c}\text { Somewhat } \\
\text { Easy }\end{array}$ & Very Easy & Total \\
\hline Baseline & $\begin{array}{c}1 \\
1.04 \%\end{array}$ & $\begin{array}{c}10 \\
10.42 \%\end{array}$ & $\begin{array}{c}9 \\
9.38 \% \\
\end{array}$ & $\begin{array}{c}20 \\
20.83 \%\end{array}$ & $\begin{array}{c}56 \\
58.33 \%\end{array}$ & 96 \\
\hline 12-Month & $\begin{array}{c}0 \\
0.00 \%\end{array}$ & $\begin{array}{c}5 \\
17.86 \%\end{array}$ & $\begin{array}{c}6 \\
21.43 \% \\
\end{array}$ & $\begin{array}{c}3 \\
10.71 \%\end{array}$ & $\begin{array}{c}14 \\
50.00 \%\end{array}$ & 28 \\
\hline Total & 1 & 15 & 15 & 23 & 70 & 124 \\
\hline
\end{tabular}

\begin{tabular}{|c|c|c|c|c|c|c|}
\hline \multicolumn{7}{|c|}{ Perception of Comfort Related to Health Seeking Behaviors } \\
\hline \multirow[t]{2}{*}{ Survey Period } & \multicolumn{6}{|c|}{$\begin{array}{l}\text { How Difficult Would It Be For You To Always Use A Method Of Birth Control To Preven } \\
\text { Pregnancy Before Sex? }\end{array}$} \\
\hline & Very Difficult & $\begin{array}{l}\text { Somewhat } \\
\text { Difficult }\end{array}$ & Neutral & $\begin{array}{l}\text { Somewhat } \\
\text { Easy }\end{array}$ & Very Easy & Total \\
\hline Baseline & $\begin{array}{c}1 \\
1.04 \%\end{array}$ & $\begin{array}{c}9 \\
9.38 \%\end{array}$ & $\begin{array}{c}13 \\
13.54 \%\end{array}$ & 14 & $\begin{array}{c}59 \\
61.46 \%\end{array}$ & 96 \\
\hline 12-Month & $\begin{array}{c}0 \\
0.00 \%\end{array}$ & $\begin{array}{c}3 \\
10.71 \%\end{array}$ & $\begin{array}{c}5 \\
17.86 \%\end{array}$ & $\begin{array}{c}5 \\
17.86 \%\end{array}$ & $\begin{array}{c}15 \\
53.57 \%\end{array}$ & 28 \\
\hline Total & 1 & 12 & 18 & 19 & 74 & 124 \\
\hline
\end{tabular}

\begin{tabular}{|c|c|c|c|c|c|c|}
\hline \multicolumn{7}{|c|}{ Perception of Comfort Related to Health Seeking Behaviors } \\
\hline \multirow[t]{2}{*}{ Survey Period } & \multicolumn{6}{|c|}{$\begin{array}{l}\text { How Difficult Would It Be For You To Go To Your Healthcare Provider In The First } 6 \\
\text { Months After Attending The FOCUS Program To Discuss Choosing A Method Of Birth } \\
\text { Control That Is Right For You? }\end{array}$} \\
\hline & Very Difficult & $\begin{array}{c}\text { Somewhat } \\
\text { Difficult }\end{array}$ & Neutral & $\begin{array}{c}\text { Somewhat } \\
\text { Easy }\end{array}$ & Very Easy & Total \\
\hline Baseline & $\begin{array}{c}5 \\
5.32 \% \\
\end{array}$ & $\begin{array}{c}7 \\
7.45 \% \\
\end{array}$ & $\begin{array}{c}22 \\
23.40 \% \\
\end{array}$ & $\begin{array}{c}12 \\
12.77 \%\end{array}$ & $\begin{array}{c}48 \\
51.06 \% \\
\end{array}$ & 94 \\
\hline 12-Month & $\begin{array}{c}2 \\
7.41 \% \\
\end{array}$ & $\begin{array}{c}4 \\
14.81 \% \\
\end{array}$ & $\begin{array}{c}4 \\
14.81 \% \\
\end{array}$ & $\begin{array}{c}4 \\
14.81 \% \\
\end{array}$ & $\begin{array}{c}13 \\
48.15 \% \\
\end{array}$ & 27 \\
\hline Total & 7 & 11 & 26 & 16 & 61 & 121 \\
\hline
\end{tabular}

\begin{tabular}{|c|c|c|c|c|c|c|}
\hline \multicolumn{7}{|c|}{ Perception of Comfort Related to Health Seeking Behaviors } \\
\hline \multirow[t]{2}{*}{ Survey Period } & \multicolumn{6}{|c|}{$\begin{array}{l}\text { How Difficult Would It Be For You To Talk To A Sexual Partner About Using A Latex } \\
\text { Condom Before Having Sex? }\end{array}$} \\
\hline & Very Difficult & $\begin{array}{l}\text { Somewhat } \\
\text { Difficult }\end{array}$ & Neutral & $\begin{array}{l}\text { Somewhat } \\
\text { Easy }\end{array}$ & Very Easy & Total \\
\hline Baseline & $\begin{array}{c}0 \\
0.00 \%\end{array}$ & $\begin{array}{c}5 \\
5.21 \%\end{array}$ & $\begin{array}{c}15 \\
15.63 \%\end{array}$ & $\begin{array}{c}15 \\
15.63 \%\end{array}$ & $\begin{array}{c}61 \\
63.54 \%\end{array}$ & 96 \\
\hline 12-Month & $\begin{array}{c}1 \\
3.57 \%\end{array}$ & $\begin{array}{c}5 \\
17.86 \%\end{array}$ & $\begin{array}{c}5 \\
17.86 \%\end{array}$ & $\begin{array}{c}5 \\
17.86 \%\end{array}$ & $\begin{array}{c}12 \\
42.86 \%\end{array}$ & 28 \\
\hline Total & 1 & 10 & 20 & 20 & 73 & 124 \\
\hline
\end{tabular}

\begin{tabular}{|c|c|c|c|c|c|c|}
\hline \multicolumn{7}{|c|}{ Perception of Comfort Related to Health Seeking Behaviors } \\
\hline \multirow[t]{2}{*}{ Survey Period } & \multicolumn{6}{|c|}{$\begin{array}{l}\text { How Difficult Would It Be For You To Go Buy Latex Condoms Or Get Them From Your } \\
\text { Healthcare Provider Before Having Sex? }\end{array}$} \\
\hline & Very Difficult & $\begin{array}{l}\text { Somewhat } \\
\text { Difficult }\end{array}$ & Neutral & $\begin{array}{l}\text { Somewhat } \\
\text { Easy }\end{array}$ & Very Easy & Total \\
\hline Baseline & $2.08 \%$ & $\begin{array}{c}9 \\
9.38 \% \\
\end{array}$ & $\begin{array}{c}14 \\
14.58 \% \\
\end{array}$ & $\begin{array}{c}18 \\
18.75 \% \\
\end{array}$ & $\begin{array}{c}53 \\
55.21 \% \\
\end{array}$ & 96 \\
\hline 12-Month & $\begin{array}{c}0 \\
0.00 \%\end{array}$ & $\begin{array}{c}2 \\
7.14 \%\end{array}$ & $\begin{array}{c}8 \\
28.57 \%\end{array}$ & $\begin{array}{c}3 \\
10.71 \%\end{array}$ & $\begin{array}{c}15 \\
53.57 \%\end{array}$ & 28 \\
\hline Total & 2 & 11 & 22 & 21 & 68 & 124 \\
\hline
\end{tabular}




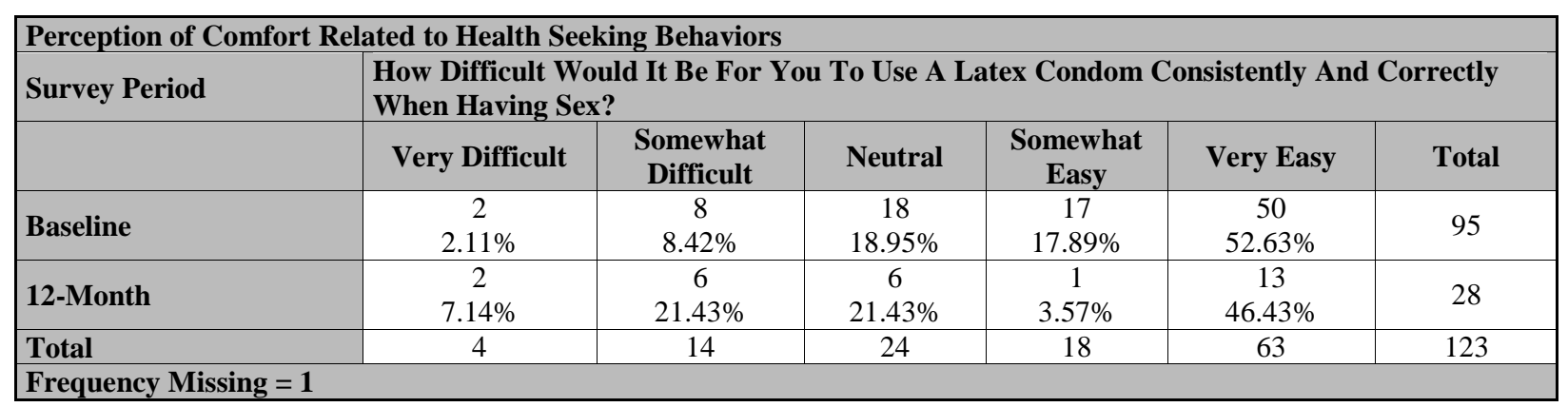

\begin{tabular}{|c|c|c|c|c|c|c|}
\hline \multicolumn{7}{|c|}{ Perception of Comfort Related to Health Seeking Behaviors } \\
\hline \multirow[t]{2}{*}{ Survey Period } & \multicolumn{6}{|c|}{$\begin{array}{l}\text { How Difficult Would It Be For You To Go To Your Healthcare Provider To Get Checked } \\
\text { For STIs If You Had Sex Without Using Condoms? }\end{array}$} \\
\hline & Very Difficult & $\begin{array}{l}\text { Somewhat } \\
\text { Difficult }\end{array}$ & Neutral & $\begin{array}{l}\text { Somewhat } \\
\text { Easy }\end{array}$ & Very Easy & Total \\
\hline Baseline & $\begin{array}{c}2 \\
2.11 \% \\
\end{array}$ & $\begin{array}{c}18 \\
18.95 \% \\
\end{array}$ & $\begin{array}{c}12 \\
12.63 \%\end{array}$ & $\begin{array}{c}19 \\
20.00 \%\end{array}$ & $\begin{array}{c}44 \\
46.32 \%\end{array}$ & 95 \\
\hline 12-Month & $0.00 \%$ & $\begin{array}{c}4 \\
14.29 \%\end{array}$ & $\begin{array}{c}4 \\
14.29 \%\end{array}$ & $\begin{array}{c}7 \\
25.00 \%\end{array}$ & $\begin{array}{c}13 \\
46.43 \%\end{array}$ & 28 \\
\hline Total & 2 & 22 & 16 & 26 & 57 & 123 \\
\hline
\end{tabular}




\section{NOTES}

